\title{
Skyrme interaction and nuclear matter constraints
}

\author{
M. Dutra, ${ }^{*}$ O. Lourenço, ${ }^{*}$ J. S. Sá Martins, and A. Delfino \\ Instituto de Física-Universidade Federal Fluminense, Avenida Litorânea s/n, 24210-150 Boa Viagem, Niterói RJ, Brazil \\ J. R. Stone \\ Oxford Physics, University of Oxford, Oxford OX1 3PU, United Kingdom and \\ Department of Physics and Astronomy, University of Tennessee, Knoxville, Tennessee 37996, USA \\ P. D. Stevenson \\ Department of Physics, University of Surrey, Guildford GU2 7XH, United Kingdom \\ (Received 16 November 2011; revised manuscript received 27 January 2012; published 5 March 2012)
}

\begin{abstract}
This paper presents a detailed assessment of the ability of the 240 Skyrme interaction parameter sets in the literature to satisfy a series of criteria derived from macroscopic properties of nuclear matter in the vicinity of nuclear saturation density at zero temperature and their density dependence, derived by the liquid-drop model, in experiments with giant resonances and heavy-ion collisions. The objective is to identify those parametrizations which best satisfy the current understanding of the physics of nuclear matter over a wide range of applications. Out of the 240 models, only 16 are shown to satisfy all these constraints. Additional, more microscopic, constraints on the density dependence of the neutron and proton effective mass $\beta$-equilibrium matter, Landau parameters of symmetric and pure neutron nuclear matter, and observational data on high- and low-mass cold neutron stars further reduce this number to 5, a very small group of recommended Skyrme parametrizations to be used in future applications of the Skyrme interaction of nuclear-matter-related observables. Full information on partial fulfillment of individual constraints by all Skyrme models considered is given. The results are discussed in terms of the physical interpretation of the Skyrme interaction and the validity of its use in mean-field models. Future work on application of the Skyrme forces, selected on the basis of variables of nuclear matter, in the Hartree-Fock calculation of properties of finite nuclei, is outlined.
\end{abstract}

DOI: 10.1103/PhysRevC.85.035201

PACS number(s): 21.30.Fe, 21.65.Cd, 21.65.Ef, 26.60.Kp

\section{INTRODUCTION}

Empirical properties of infinite nuclear matter can be calculated using many different theoretical approaches. The most microscopic ones start from a realistic two-body free nucleon-nucleon $(N N)$ interaction with parameters fitted to $N N$ scattering phase shifts in different partial wave channels and to properties of the deuteron [1]. By taking these bare $N N$ interactions as input into a many-body formalism, such as the relativistic Dirac-Bruckner-Hartree-Fock (DBHF) approximation and its nonrelativistic counterpart BHF [2,3], variational methods [4,5], correlated basis function models [6], selfconsistent Green's function (SCGF) models [7,8], quantum Monte Carlo techniques [9-14], and chiral effective field theory $[15,16]$, an effective $N N$ interaction, which includes the effect of the medium, is derived and the many-body problem is approximately solved.

Various many-body approaches typically lead to an overprediction of the saturation density $\rho_{\mathrm{o}}$ of symmetric nuclear matter (SNM), at which the binding energy per nucleon reaches its maximum, and of the corresponding maximum binding energy $E_{0}\left(\rho=\rho_{0}\right)$ [17]. There are many ways of estimating the experimental value of $\rho_{\mathrm{o}}$, including different variants

\footnotetext{
*Present address: Departamento de Física, Instituto Tecnológico da Aeronáutica, CTA, São José dos Campos, 12228-900 SP, Brazil.
}

of the liquid-drop models, optical model of $N N$ scattering, muonic atoms, and Hartree-Fock (HF) calculation of nuclear density distributions (see, e.g., [18] and references therein). The range of results is rather broad but a consensus value is $\rho_{\mathrm{o}}=0.17 \pm 0.03 \mathrm{fm}^{-3}$. The empirical value $E_{\mathrm{o}}$ per nucleon of $\sim 16 \mathrm{MeV}$ can be extracted from the semiempirical mass formula or from the extrapolation of binding energies of heavy nuclei. Theoretical calculation of saturation properties of SNM depends not only on the choice of the bare $N N$ interaction but also on the method of treatment of the many-body effects. For example, if the BHF approximation is used, $E_{\mathrm{o}}$ and $\rho_{\mathrm{o}}$ are correlated within a narrow band $[17,19]$. Two main approaches have been suggested to improve the theoretical calculation of saturation properties of SNM, the most frequently used being the inclusion of three-body ( $N N N$ ) forces. As the form of these forces is unknown, different ad hoc parametrizations have been used, dependent on additional variable parameters that need to be fitted to account for the delicate balance between the strong $(N N N)$ attraction and $(N N)$ repulsion at short distances. Alternatively, DBHF calculations have been shown to be effective without the need for $N N N$ forces [17,20]. Another possibility is to treat the scalar and vector densities in the Walecka relativistic mean-field model [21] as equal [22]. However, the systematic deviation of all theoretical predictions from the expected empirical values of $E_{\mathrm{o}}$ and $\rho_{\mathrm{o}}$ remains a problem. An interesting suggestion by Dewulf et al. [7] implies that treatment of short-range correlations in nuclear matter in the SCGF model brings the saturation density closer to the empirical value than do current BHF calculations. 
Careful examination of the effect of long-range correlations on nuclear saturation properties through coherent pion-exchange contributions to the binding energy of nuclear matter is equally important. Such correlations are not present in finite nuclei and a question arises as to the applicability of liquid-drop model predictions, based on properties of finite nuclei, to saturation properties of nuclear matter.

Quite a different perspective on treating many-nucleon systems is to use effective density-dependent $N N$ and $N N N$ interactions instead of realistic ones. The pioneering models of Köhler [23], Brink and Boeker [24], Moszkowski [25], and Skyrme [26], further developed by Vautherin and Brink (see [27] and references therein) and Gogny [28], initiated this approach, which is widely used today. The basic idea is to parametrize the $N N$ and $N N N$ interactions by zerorange (Skyrme model), short finite-range (Gogny model), and indefinite-range (separable monopole model (SMO) [29,30]) density-dependent functionals to describe the ground-state properties of finite nuclei and nuclear matter. In this scenario, the microscopic details of $N N$ and $N N N$ forces, such as meson exchange, are not explicitly considered and all the physically relevant information is carried by the parameters of the density-dependent phenomenological forces, which include the spin, orbital angular momentum, and isospin couplings. The drawback of this approach is that the parametrization of such forces is not unique and there exist, in principle, an infinite number of parameter sets, fitted to ground-state properties of (doubly or semimagic) stable nuclei, fission barriers, energies of giant resonances, and symmetric and asymmetric nuclear matter (ANM). This situation arises in part because there is no unambiguous connection between individual parameters, or groups of parameters, of these forces with particular physical properties of the many-body nuclear system. Many parameters are strongly correlated.

It is obviously desirable to constrain the parametrizations of effective density-dependent forces as much as possible. The strategy chosen in this work is to concentrate first on application of the forces to modeling different variants of infinite nuclear matter. Although nuclear matter is an idealized medium, and all its properties, derived from experiments indirectly in a model-dependent way, are empirical quantities, it offers an important insight into specific parts of the phenomenological interaction and has important applications in the theory of heavy-ion collisions (HIC) and the physics of neutron stars. This paper focuses on the Skyrme interaction and is the first part of a series in which nuclear matter constraints will be applied to each class of effective density-dependent interaction. It will be followed by investigation of Gogny and SMO forces and interactions used in relativistic mean-field (RMF) models. After this work is complete, the implications of the consequences for the theory of finite nuclei will be investigated.

We use the most up-to-date constraints on nuclear matter properties which go much beyond the minimal conditions on SNM (saturation density, binding energy, incompressibility, and symmetry energy at saturation). New data from HIC, giant monopole, and dipole resonance experiments as well as new observational data on neutron stars provide new constraints on the performance of individual Skyrme parametrizations in nuclear matter.
The set of eleven macroscopic constraints used in this work have been mainly derived from experimental data, on the assumption of the validity of the liquid-drop model [31,32], and concern properties of SNM at and close to the saturation point. Studies of dilute Fermi gases provide constraints on the low-density pure neutron matter (PNM) equation of state (EoS). Dynamical models of HIC further constrain the density dependence of pressure in SNM and PNM at subsaturation density and extrapolate these constraints to higher densities. Use of the mean-field Hartree-Fock + random-phase approximation (RPA) for calculating giant resonance excitation energies both in relativistic and nonrelativistic models provide a final group of constraints on the incompressibility of nuclear matter and its density and symmetry dependence.

In addition to the above macroscopic constraints, several more microscopic constraints are employed. These include the density dependence of the nucleon effective masses in $\beta$-equilibrium matter (BEM), the Landau parameters for SNM and PNM, and the symmetry energy. Observational data on cold nonrotational high-mass and low-mass neutron stars provide a final group of constraints.

In this work we consider 240 Skyrme parameter sets, currently available in the literature, and critically compare their predictions for a wide variety of properties of SNM, PNM, asymmetric matter with fixed ratio proton fraction (ANM), and BEM with all available constraints in the density range from $\sim 0.1 \rho_{\mathrm{o}}$ to $3 \rho_{\mathrm{o}}$, estimated on the best available experimental and theoretical grounds. The range of applicability of the Skyrme force is a very important issue which is often mishandled.

The Skyrme interaction, originally constructed for finite nuclei and nuclear matter at saturation density, is a lowmomentum expansion of the effective two-body $N N$ interaction in momentum space, and neither the lower nor upper limit of its validity has been firmly established. The important point about the Skyrme interaction is that some correlation effects are included through its parameters. Thus, although formulated as a zero-range interaction in coordinate space [27], it exhibits some finite-range features [33].

For finite nuclei, the best evidence for a lower limit derives from the fact that Skyrme models reasonably predict the observed abrupt decrease of density at the nuclear surface and neutron and proton mean-square radii. The sensitivity extends down to about $0.1 \rho_{\mathrm{o}}$.

In uniform SNM, the Skyrme interaction has been used to make prediction of the appearance of light clusters (deuterons, tritons, ${ }^{3} \mathrm{He}$, and $\alpha$ particles in hot matter in the region of density of $0.6 \rho_{\mathrm{o}}-1.25 \rho_{\mathrm{o}}$ [34]). More recently, the abundance of light clusters with $A \leqslant 13$ in supernova envelopes at finite temperature was calculated in the density range $0.01 \rho_{\mathrm{o}}-0.5 \rho_{\mathrm{o}}$ using the Skyrme functional [35]. The "pasta" phase, predicted in neutron star and supernova matter in a variety of models $[36,37]$, was successfully modeled in supernova matter in the density range $0.25 \rho_{\mathrm{o}}-0.75 \rho_{\mathrm{o}}$ in a self-consistent $\mathrm{HF}+\mathrm{BCS}$ calculation with $\mathrm{SkM}^{*}$ and SLy4 Skyrme interactions [38].

PNM has been mainly studied as an approximation to a low-density Fermi gas. Schwenk and Pethick [39] explored, in a model-independent way, the neutron matter EoS at densities $0.0125 \rho_{\mathrm{o}}-0.125 \rho_{\mathrm{o}}$, and Epelbaum et al. [40] calculated the 
ground-state energy of dilute neutron matter at next-toleading-order in lattice chiral effective field theory in the density range $0.02 \rho_{\mathrm{o}}-0.1 \rho_{\mathrm{o}}$. Quantum Monte Carlo techniques have been applied to low-density PNM, providing a constraint on the EoS up to saturation density [41,42]. To our knowledge, there has not yet been a detailed study of the applicability of the Skyrme interaction at these low densities in PNM. Such a study is of particular interest as it may be one of the best ways to model the crust of neutron stars.

The upper density limit of validity of the Skyrme interaction reflects the fact that at higher densities relativistic effects should be increasingly important. The appearance of heavy strange baryons and mesons in the matter is ultimately inevitable. Due to Pauli blocking, the chemical potential of the neutrons increases rapidly with density. At some point, it becomes energetically favorable for the system to let the neutrons undergo a strangeness-changing weak decay, which replaces them by hyperons, for which the Fermi sea is not yet filled. From the difference of mass between the neutron and its strange partners it follows that the critical density, at which hyperons should appear, is $2 \rho_{\mathrm{o}}-3 \rho_{\mathrm{o}}$. Using a nucleon-only Skyrme interaction beyond this density can be expected to yield misleading results. This is discussed later in connection with high-mass neutron star models.

Taking all the above pieces of evidence into account, we adopt $0.01 \rho_{\mathrm{o}} \leqslant \rho \leqslant 3 \rho_{\mathrm{o}}$ as the range of validity of the Skyrme interactions considered in this work.

The paper is organized as follows. A brief description of the Skyrme interaction, together with definition of the variables used in this work, is given in Sec. II. Classification of the macroscopic constraints and discussion of their origin and applicability range form the contents of Sec. III. Section IV presents a comparison of predictions of those Skyrme parametrizations which satisfy the macroscopic constraints with further microscopic and observational constraints. The results are discussed and summarized in Sec. V and conclusions are presented in Sec. VI.

\section{SKYRME MODELS}

Since the original work by Skyrme in the 1950s [26] and the Vautherin and Brink [27] parametrization of the original interaction in the early 1970s, considerable effort has been invested in the application of this density-dependent effective interaction to both ground-state properties of finite nuclei and nuclear matter in the framework of the mean-field Hartree-Fock approximation (see, e.g., [43,44] for recent reviews). The advantage of the structure of the Skyrme density functional is that it allows analytical expression of all variables characterizing infinite nuclear matter [45-47]. Such structure can also be constructed from nonrelativistic versions of the relativistic point-coupling models [48-50]. In the following, we introduce the various physical quantities and give expressions for each in terms of the Skyrme parameters. The general expression for the energy per particle of infinite ANM, defined in terms of the energy density $\mathcal{E}$ and particle number density $\rho$, is given as

$$
\begin{aligned}
E=\frac{\mathcal{E}}{\rho}= & \frac{3 \hbar^{2}}{10 M}\left(\frac{3 \pi^{2}}{2}\right)^{2 / 3} \rho^{2 / 3} H_{5 / 3}+\frac{t_{0}}{8} \rho\left[2\left(x_{0}+2\right)-\left(2 x_{0}+1\right) H_{2}\right]+\frac{1}{48} \sum_{i=1}^{3} t_{3 i} \rho^{\sigma_{i}+1}\left[2\left(x_{3 i}+2\right)-\left(2 x_{3 i}+1\right) H_{2}\right] \\
& +\frac{3}{40}\left(\frac{3 \pi^{2}}{2}\right)^{2 / 3} \rho^{5 / 3}\left(a H_{5 / 3}+b H_{8 / 3}\right)+\frac{3}{40}\left(\frac{3 \pi^{2}}{2}\right)^{2 / 3} \rho^{5 / 3+\delta}\left[t_{4}\left(x_{4}+2\right) H_{5 / 3}-t_{4}\left(x_{4}+\frac{1}{2}\right) H_{8 / 3}\right] \\
& +\frac{3}{40}\left(\frac{3 \pi^{2}}{2}\right)^{2 / 3} \rho^{5 / 3+\gamma}\left[t_{5}\left(x_{5}+2\right) H_{5 / 3}+t_{5}\left(x_{5}+\frac{1}{2}\right) H_{8 / 3}\right],
\end{aligned}
$$

with

$$
\begin{aligned}
a & =t_{1}\left(x_{1}+2\right)+t_{2}\left(x_{2}+2\right), \\
b & =\frac{1}{2}\left[t_{2}\left(2 x_{2}+1\right)-t_{1}\left(2 x_{1}+1\right)\right], \\
H_{n}(y) & =2^{n-1}\left[y^{n}+(1-y)^{n}\right],
\end{aligned}
$$

where $y=Z / A$ is the proton fraction. Equation (1) includes the summation over index $i$ in the third term introduced by Agrawal et al. [51] and additional terms involving $t_{4}, x_{4}, t_{5}$, and $x_{5}$, used by Chamel et al. [52]. The great majority of the parametrizations referred to in this work do not include these terms. Parametrizations without (with) these additional terms are regarded as "standard" ("nonstandard") in this paper. We note that there are several other parameter sets which parametrize the density dependence of the Skyrme functional in nonstandard ways [53-56], different from those considered here. These forces have been reported to have problems at higher density nuclear matter [51] and have not been included in the present study.

All quantities referred to in this work have been obtained based on Eq. (1) and are given below.

Equation (1) leads to an in-medium effective nucleon mass $M^{*}$ in ANM of

$$
M^{*}=M\left\{H_{5 / 3}+\frac{1}{4} \frac{M}{\hbar^{2}} \rho\left[\left(a+t_{4}\left(x_{4}+2\right) \rho^{\delta}+t_{5}\left(x_{5}+2\right) \rho^{\gamma}\right) H_{5 / 3}+\left(b-t_{4}\left(x_{4}+\frac{1}{2}\right) \rho^{\delta}+t_{5}\left(x_{5}+\frac{1}{2}\right) \rho^{\gamma}\right) H_{8 / 3}\right]\right\}^{-1}
$$

with $M$ being the free nucleon mass. 
The pressure, defined as $P=\rho^{2} \frac{\partial(\mathcal{E} / \rho)}{\partial \rho}$, is given as

$$
\begin{aligned}
P= & \frac{\hbar^{2}}{5 M}\left(\frac{3 \pi^{2}}{2}\right)^{2 / 3} \rho^{5 / 3} H_{5 / 3}+\frac{t_{0}}{8} \rho^{2}\left[2\left(x_{0}+2\right)-\left(2 x_{0}+1\right) H_{2}\right]+\frac{1}{48} \sum_{i=1}^{3} t_{3 i}\left(\sigma_{i}+1\right) \rho^{\sigma_{i}+2}\left[2\left(x_{3 i}+2\right)-\left(2 x_{3 i}+1\right) H_{2}\right] \\
& +\frac{1}{8}\left(\frac{3 \pi^{2}}{2}\right)^{2 / 3} \rho^{8 / 3}\left(a H_{5 / 3}+b H_{8 / 3}\right)+\frac{1}{40}\left(\frac{3 \pi^{2}}{2}\right)^{2 / 3}(5+3 \delta) \rho^{\frac{8}{3}+\delta}\left[t_{4}\left(x_{4}+2\right) H_{5 / 3}-t_{4}\left(x_{4}+\frac{1}{2}\right) H_{8 / 3}\right] \\
& +\frac{1}{40}\left(\frac{3 \pi^{2}}{2}\right)^{2 / 3}(5+3 \gamma) \rho^{\frac{8}{3}+\gamma}\left[t_{5}\left(x_{5}+2\right) H_{5 / 3}+t_{5}\left(x_{5}+\frac{1}{2}\right) H_{8 / 3}\right] .
\end{aligned}
$$

The volume incompressibility of ANM at saturation density is calculated as the derivative of pressure with respect to the number density $\rho$ :

$$
\begin{aligned}
K= & 9 \rho^{2}\left(\frac{\partial^{2} \mathcal{E} / \rho}{\partial \rho^{2}}\right)=9\left(\frac{\partial P}{\partial \rho}\right)=\frac{3 \hbar^{2}}{M}\left(\frac{3 \pi^{2}}{2}\right)^{2 / 3} \rho^{2 / 3} H_{5 / 3}+\frac{9 t_{0}}{4} \rho\left[2\left(x_{0}+2\right)-\left(2 x_{0}+1\right) H_{2}\right] \\
& +\frac{3}{16} \sum_{i=1}^{3} t_{3 i}\left(\sigma_{i}+1\right)\left(\sigma_{i}+2\right) \rho^{\sigma_{i}+1}\left[2\left(x_{3 i}+2\right)-\left(2 x_{3 i}+1\right) H_{2}\right]+3\left(\frac{3 \pi^{2}}{2}\right)^{2 / 3} \rho^{5 / 3}\left(a H_{5 / 3}+b H_{8 / 3}\right) \\
& +\frac{3}{40}\left(\frac{3 \pi^{2}}{2}\right)^{2 / 3}(5+3 \delta)(8+3 \delta) \rho^{\frac{5}{3}+\delta}\left[t_{4}\left(x_{4}+2\right) H_{5 / 3}-t_{4}\left(x_{4}+\frac{1}{2}\right) H_{8 / 3}\right] \\
& +\frac{3}{40}\left(\frac{3 \pi^{2}}{2}\right)^{2 / 3}(5+3 \gamma)(8+3 \gamma) \rho^{\frac{5}{3}+\gamma}\left[t_{5}\left(x_{5}+2\right) H_{5 / 3}+t_{5}\left(x_{5}+\frac{1}{2}\right) H_{8 / 3}\right] .
\end{aligned}
$$

Finally, the third derivative of the energy per particle in ANM at saturation density, also called the skewness coefficient, is expressed as

$$
\begin{aligned}
Q= & 27 \rho^{3}\left(\frac{\partial^{3} \mathcal{E} / \rho}{\partial \rho^{3}}\right)=\frac{12 \hbar^{2}}{5 M}\left(\frac{3 \pi^{2}}{2}\right)^{2 / 3} \rho^{2 / 3} H_{5 / 3}+\frac{9}{16} \sum_{i=1}^{3} t_{3 i} \sigma_{i}\left(\sigma_{i}+1\right)\left(\sigma_{i}-1\right) \rho^{\sigma_{i}+1}\left[2\left(x_{3 i}+2\right)-\left(2 x_{3 i}+1\right) H_{2}\right] \\
& -\frac{3}{4}\left(\frac{3 \pi^{2}}{2}\right)^{2 / 3} \rho^{5 / 3}\left(a H_{5 / 3}+b H_{8 / 3}\right)+\frac{3}{40}\left(\frac{3 \pi^{2}}{2}\right)^{2 / 3}(2+3 \delta)(5+3 \delta)(3 \delta-1) \rho^{\frac{5}{3}+\delta}\left[t_{4}\left(x_{4}+2\right) H_{5 / 3}-t_{4}\left(x_{4}+\frac{1}{2}\right) H_{8 / 3}\right] \\
& +\frac{3}{40}\left(\frac{3 \pi^{2}}{2}\right)^{2 / 3}(2+3 \gamma)(5+3 \gamma)(3 \gamma-1) \rho^{\frac{5}{3}+\gamma}\left[t_{5}\left(x_{5}+2\right) H_{5 / 3}+t_{5}\left(x_{5}+\frac{1}{2}\right) H_{8 / 3}\right] .
\end{aligned}
$$

In a special case for SNM $(y=1 / 2)$, expressions (1)-(8) simplify in that $H_{\mathrm{n}}=1$ and we obtain the energy per particle, $E_{\mathrm{SNM}}(\rho)$, the incompressibility $K_{\mathrm{SNM}}(\rho)$, and the skewness $Q_{\mathrm{SNM}}(\rho)$. When calculating properties of symmetric matter at the saturation density, $E_{\mathrm{SNM}}\left(\rho_{\mathrm{o}}\right)=E_{\mathrm{o}}, K_{\mathrm{SNM}}\left(\rho_{\mathrm{o}}\right)=K_{\mathrm{o}}$ and $Q_{\mathrm{SNM}}\left(\rho_{\mathrm{o}}\right)=Q_{\mathrm{o}}$, the second and third derivatives of the energy density with respect to number density are taken at $\rho=\rho_{\mathrm{o}}$. Obviously, the first derivative, the pressure, is equal to zero at $\rho_{\mathrm{o}}$.

One of the key properties of nuclear matter is the symmetry energy, which is particularly important in modeling nuclear matter and finite nuclei because it probes the isospin part of the Skyrme interaction. It is defined as

$$
\begin{aligned}
\mathcal{S}(\rho) & =\left.\frac{1}{8} \frac{\partial^{2}(\mathcal{E} / \rho)}{\partial y^{2}}\right|_{\rho, y=1 / 2}=\frac{\hbar^{2}}{6 M}\left(\frac{3 \pi^{2}}{2}\right)^{2 / 3} \rho^{2 / 3}-\frac{t_{0}}{8}\left(2 x_{0}+1\right) \rho-\frac{1}{48} \sum_{i=1}^{3} t_{3 i}\left(2 x_{3 i}+1\right) \rho^{\sigma_{i}+1} \\
& +\frac{1}{24}\left(\frac{3 \pi^{2}}{2}\right)^{2 / 3}[a+4 b] \rho^{5 / 3}-\frac{1}{8}\left(\frac{3 \pi^{2}}{2}\right)^{2 / 3} t_{4} x_{4} \rho^{\frac{5}{3}+\delta}+\frac{1}{24}\left(\frac{3 \pi^{2}}{2}\right)^{2 / 3} t_{5}\left(5 x_{5}+4\right) \rho^{\frac{5}{3}+\gamma} .
\end{aligned}
$$

In SNM, it is customary to define four quantities, $J=\mathcal{S}\left(\rho_{\mathrm{o}}\right), L, K_{\text {sym }}$, and $Q_{\text {sym }}$, related to the symmetry energy and its derivatives evaluated at the saturation density $\rho_{\mathrm{o}} . L$, the slope of $\mathcal{S}$, is given by

$$
\begin{aligned}
L= & 3 \rho_{\mathrm{o}}\left(\frac{\partial \mathcal{S}}{\partial \rho}\right)_{\rho=\rho_{\mathrm{o}}}=\frac{\hbar^{2}}{3 M}\left(\frac{3 \pi^{2}}{2}\right)^{2 / 3} \rho_{\mathrm{o}}^{2 / 3}-\frac{3 t_{0}}{8}\left(2 x_{0}+1\right) \rho_{\mathrm{o}}-\frac{1}{16} \sum_{i=1}^{3} t_{3 i}\left(2 x_{3 i}+1\right)\left(\sigma_{i}+1\right) \rho_{\mathrm{o}}^{\sigma_{i}+1} \\
& +\frac{5}{24}\left(\frac{3 \pi^{2}}{2}\right)^{2 / 3}(a+4 b) \rho_{\mathrm{o}}^{5 / 3}-\frac{1}{8}\left(\frac{3 \pi^{2}}{2}\right)^{2 / 3}(5+3 \delta) t_{4} x_{4} \rho_{\mathrm{o}}^{\frac{5}{3}+\delta}+\frac{1}{24}\left(\frac{3 \pi^{2}}{2}\right)^{2 / 3}(5+3 \gamma) t_{5}\left(5 x_{5}+4\right) \rho_{\mathrm{o}}^{\frac{5}{3}+\gamma} .
\end{aligned}
$$


The curvature of the symmetry energy $\mathcal{S}$ at saturation density in SNM is sometimes called the symmetry incompressibility, $K_{\text {sym }}$. It should not be confused with $K_{\tau}$, which is the isospin incompressibility, defined in Eqs. (21) and (24). $K_{\text {sym }}$ is given by

$$
\begin{aligned}
K_{\text {sym }}= & 9 \rho_{\mathrm{o}}^{2}\left(\frac{\partial^{2} \mathcal{S}}{\partial \rho^{2}}\right)_{\rho=\rho_{0}}=-\frac{\hbar^{2}}{3 M}\left(\frac{3 \pi^{2}}{2}\right)^{2 / 3} \rho_{\mathrm{o}}^{2 / 3}-\frac{3}{16} \sum_{i=1}^{3} t_{3 i}\left(2 x_{3 i}+1\right)\left(\sigma_{i}+1\right) \sigma_{i} \rho_{\mathrm{o}}^{\sigma_{i}+1}+\frac{5}{12}\left(\frac{3 \pi^{2}}{2}\right)^{2 / 3}(a+4 b) \rho_{\mathrm{o}}^{5 / 3} \\
& -\frac{1}{8}\left(\frac{3 \pi^{2}}{2}\right)^{2 / 3}(5+3 \delta)(2+3 \delta) t_{4} x_{4} \rho_{\mathrm{o}}^{\frac{5}{3}+\delta}+\frac{1}{24}\left(\frac{3 \pi^{2}}{2}\right)^{2 / 3}(5+3 \gamma)(2+3 \gamma) t_{5}\left(5 x_{5}+4\right) \rho_{\mathrm{o}}^{\frac{5}{3}+\gamma} .
\end{aligned}
$$

Finally, $Q_{\text {sym }}$, the third derivative of the symmetry energy, is

$$
\begin{aligned}
Q_{\text {sym }}= & 27 \rho_{\mathrm{o}}^{3}\left(\frac{\partial^{3} \mathcal{S}}{\partial \rho^{3}}\right)_{\rho=\rho_{0}}=\frac{4 \hbar^{2}}{3 M}\left(\frac{3 \pi^{2}}{2}\right)^{2 / 3} \rho_{\mathrm{o}}^{2 / 3}-\frac{9}{16} \sum_{i=1}^{3} t_{3 i}\left(2 x_{3 i}+1\right)\left(\sigma_{i}+1\right) \sigma_{i}\left(\sigma_{i}-1\right) \rho_{\mathrm{o}}^{\sigma_{i}+1}-\frac{5}{12}\left(\frac{3 \pi^{2}}{2}\right)^{2 / 3}(a+4 b) \rho_{\mathrm{o}}^{5 / 3} \\
& -\frac{1}{8}\left(\frac{3 \pi^{2}}{2}\right)^{2 / 3}(5+3 \delta)(2+3 \delta)(3 \delta-1) t_{4} x_{4} \rho_{\mathrm{o}}^{\frac{5}{3}+\delta}+\frac{1}{24}\left(\frac{3 \pi^{2}}{2}\right)^{2 / 3}(5+3 \gamma)(2+3 \gamma)(3 \gamma-1) t_{5}\left(5 x_{5}+4\right) \rho_{\mathrm{o}}^{\frac{5}{3}+\gamma}
\end{aligned}
$$

By using the above expressions, the density dependence of the symmetry energy can be expanded as a function of $x=\left(\rho-\rho_{0}\right) / 3 \rho_{0}$ :

$$
\mathcal{S}=J+L x+\frac{1}{2} K_{\mathrm{sym}} x^{2}+\frac{1}{6} Q_{\mathrm{sym}} x^{3}+O\left(x^{4}\right) .
$$

Similarly, in SNM, the density dependence of the energy per particle, $E$, Eq. (1), is sometimes expressed as an expansion in a Taylor series around $\rho_{\mathrm{o}}$ :

$$
E_{\mathrm{SNM}}(\rho)=E_{\mathrm{o}}+\frac{1}{2} K_{\mathrm{o}} x^{2}+\frac{1}{6} Q_{\mathrm{o}} x^{3}+O\left(x^{4}\right),
$$

where $E_{0}=E_{\mathrm{SNM}}\left(\rho_{0}\right)$ is the energy per particle at the saturation density $\rho_{\mathrm{o}}$ and

$$
K_{\mathrm{o}}=9 \rho_{\mathrm{o}}^{2}\left(\frac{\partial^{2} E_{\mathrm{SNM}}(\rho)}{\partial \rho^{2}}\right)_{\rho=\rho_{\mathrm{o}}}
$$

and

$$
Q_{\mathrm{o}}=27 \rho_{\mathrm{o}}^{3}\left(\frac{\partial^{3} E_{\mathrm{SNM}}(\rho)}{\partial \rho^{3}}\right)_{\rho=\rho_{\mathrm{o}}}
$$

In ANM with asymmetry $\beta=(N-Z) / A=(1-2 y)$, the energy per particle, $E$, can be expanded around a new, isospindependent saturation density $\rho_{\mathrm{o}}(\beta) \sim \rho_{\mathrm{o}}\left[1-3\left(L / K_{\mathrm{o}}\right) \beta^{2}\right]$ [57]:

$$
\begin{aligned}
E_{\mathrm{ANM}}(\rho, \beta)= & E_{\mathrm{o}}\left(\rho_{\mathrm{o}}(\beta)\right)+\frac{K_{\mathrm{o}}\left(\rho_{\mathrm{o}}(\beta)\right)}{2}\left(\frac{\rho-\rho_{\mathrm{o}}(\beta)}{3 \rho_{\mathrm{o}}(\beta)}\right)^{2} \\
& +\frac{Q_{\mathrm{o}}\left(\rho_{\mathrm{o}}(\beta)\right)}{6}\left(\frac{\rho-\rho_{\mathrm{o}}(\beta)}{3 \rho_{\mathrm{o}}(\beta)}\right)^{3}+O\left(\beta^{4}\right),
\end{aligned}
$$

where the expansion coefficients are given as

$$
\begin{aligned}
& E_{\mathrm{o}}\left(\rho_{\mathrm{o}}(\beta)\right)=E_{\mathrm{o}}+J \beta^{2}+O\left(\beta^{4}\right), \\
& K_{\mathrm{o}}\left(\rho_{\mathrm{o}}(\beta)\right)=K_{\mathrm{o}}+\left(K_{\mathrm{sym}}-6 L-\frac{Q_{\mathrm{o}}}{K_{\mathrm{o}}} L\right) \beta^{2}+O\left(\beta^{4}\right), \\
& Q_{\mathrm{o}}\left(\rho_{\mathrm{o}}(\beta)\right)=Q_{\mathrm{o}}+\left(Q_{\mathrm{sym}}-9 L \frac{Q_{\mathrm{o}}}{K_{\mathrm{o}}}\right) \beta^{2}+O\left(\beta^{4}\right) .
\end{aligned}
$$

The coefficient of the second term in Eq. (19),

$$
K_{\tau, v}=\left(K_{\mathrm{sym}}-6 L-\frac{Q_{\mathrm{o}}}{K_{\mathrm{o}}} L\right),
$$

determines the isospin dependence of incompressibility at saturation density $\rho_{\mathrm{o}}(\beta)$. Strictly, it is the volume part, $K_{\tau, v}$, of the isospin incompressibility $K_{\tau}$, Eq. (24), which plays an important role in analysis of data from giant monopole resonances. It does not include surface effects, as discussed in Secs. III A and III C.

\section{MACROSCOPIC CONSTRAINTS}

It is important to keep in mind that different Skyrme parametrizations were often constructed with emphasis on a certain selection of data on finite nuclei. For example, the BSk family members were fitted to experimental nuclear masses, $\mathrm{SkM}^{*}$ to binding energies of finite nuclei and actinide fission barriers, the SkI family to isotope shifts in the $\mathrm{Pb}$ region, and the SLy family to properties of neutron matter, neutron stars, and the ground-state variables of neutron-heavy nuclei. Although all Skyrme forces are usually fitted to reproduce well the saturation energy and density of symmetric nuclear matter, they differ significantly in other characteristics of symmetric and pure neutron matter, in particular their density dependence.

We examine in this section eleven constraints on properties of nuclear matter, out of which four are related to SNM, two to PNM, and five to both SNM and PNM. The constraints are listed in Table I.

\section{A. Symmetric nuclear matter}

Infinite nuclear matter, composed of the same number of protons and neutrons without Coulomb interaction, does not exist in nature. Nevertheless, it has become an important theoretical laboratory for the investigation of physical quantities relevant for the modeling of heavy nuclei and nuclear matter in astrophysical compact objects. As stated above, the saturation density $\rho_{\mathrm{o}}$ and the binding energy per nucleon, $E_{0}=\mathcal{E} / \rho_{\mathrm{o}}$, are reasonably well established. In this work we focus on two 
TABLE I. List of macroscopic constraints and the range of their experimental (exp) and empirical (emp) values, density region in which they are valid, and the corresponding range as found using successful Skyrme parametrizations (CSkP). For more explanation see text.

\begin{tabular}{|c|c|c|c|c|c|c|}
\hline SM1 & $K_{\mathrm{o}}$ & (7),(15) & $\rho_{\mathrm{o}}\left(\mathrm{fm}^{-3}\right)$ & $200-260 \mathrm{MeV}$ & $202.0-240.3 \mathrm{MeV}$ & {$[64]$} \\
\hline SM3 & $P(\rho)$ & (6) & $2<\frac{\rho}{\rho_{0}}<3$ & Band region & see Fig. 1 & {$[78]$} \\
\hline SM4 & $P(\rho)$ & (6) & $1.2<\frac{\rho}{\rho_{0}}<2.2$ & Band region & see Fig. 2 & {$[80]$} \\
\hline PNM2 & $P(\rho)$ & (6) & $2<\frac{\rho}{\rho_{0}}<3$ & Band region & see Fig. 5 & [78] \\
\hline MIX1 & $J$ & (9) & $\rho_{\mathrm{o}}\left(\mathrm{fm}^{-3}\right)$ & $30-35 \mathrm{MeV}$ & $30.0-35.5 \mathrm{MeV}$ & [44] \\
\hline MIX2 & $L$ & (10) & $\rho_{\mathrm{o}}\left(\mathrm{fm}^{-3}\right)$ & $40-76 \mathrm{MeV}$ & 48.6-67.1 MeV & [101] \\
\hline MIX3 & $K_{\tau, \mathrm{v}}$ & (21) & $\rho_{\mathrm{o}}\left(\mathrm{fm}^{-3}\right)$ & -760 to $-372 \mathrm{MeV}$ & -407.1 to $-360.1 \mathrm{MeV}$ & [107] \\
\hline MIX4 & $\frac{\mathcal{S}\left(\rho_{0} / 2\right)}{J}$ & - & $\rho_{\mathrm{o}}\left(\mathrm{fm}^{-3}\right)$ & $0.57-0.86$ & $0.61-0.67$ & [110] \\
\hline
\end{tabular}

other physical quantities of SNM at saturation density: the incompressibility $K_{\mathrm{o}}$ and the skewness coefficient $Q_{\mathrm{o}}$. We note that some authors use $K^{\prime}=-Q_{\mathrm{o}}$ as the skewness coefficient [see Eq. (8)].

The determination of $K_{\mathrm{o}}$, along with other related parameters of nuclear matter from experimental data on the giant monopole resonance (GMR) on finite nuclei, has been a long-standing problem (see, e.g., [58-60]) which has not been fully resolved to this day [61].

There are basically two procedures that can be used to extract the information. One is to analyze the $A$ dependence of the compression modulus of a finite nucleus,

$$
K_{\mathrm{A}}=\left(M / \hbar^{2}\right)\left\langle R^{2}\right\rangle E_{\mathrm{GMR}}^{2},
$$

obtained, using sum-rule arguments, from the measured energy of GMR $E_{\mathrm{GMR}}$ in spherical nuclei [58]. Here $M$ is the nucleon mass and $R$ is the rms matter radius of the nucleus with mass number $A$. Using the leptodermous expansion of the energy per particle in the droplet model (the mass formula) leads to the following expression for $K_{\mathrm{A}}$ [58]:

$$
\begin{aligned}
K_{\mathrm{A}}= & K_{\mathrm{vol}}+K_{\text {surf }} A^{-1 / 3}+K_{\text {curv }} A^{-2 / 3}+K_{\tau} \beta^{2} \\
& +K_{\text {coul }} \frac{Z^{2}}{A^{4 / 3}}+\cdots,
\end{aligned}
$$

where the isospin incompressibility $K_{\tau}$ consists of two components [58,62,63],

$$
K_{\tau}=K_{\tau, v}+K_{\tau, s} A^{-1 / 3},
$$

where $K_{\tau, \mathrm{v}}$ and $K_{\tau, s}$ determine the volume and surface isospin incompressibility, respectively. $K_{\text {vol }}, K_{\text {surf }}, K_{\text {curv }}, K_{\tau}$, and $K_{\text {coul }}$ are second derivatives of the coefficients of the volume, surface, curvature, isospin, and Coulomb terms with respect to the radial coordinate of the nucleus in the mass formula. If the $K_{\mathrm{vol}}$ term is identified as the incompressibility of infinite nuclear matter, $K_{\mathrm{o}}$, then a link between $K_{\mathrm{A}}$ and $K_{\mathrm{o}}$ can be used to determine $K_{\mathrm{o}}$. However, it has been argued that the macroscopic analysis suffers from potentially serious drawbacks, including the uncertainty of the validity of the $K_{\mathrm{vol}} \approx K_{\mathrm{o}}$ assumption, the weak $A$ dependence, limitations of Eq. (23) to small oscillations, questions about convergence of the expansion in Eq. (23), and consideration of possible anharmonicities of the breathing mode, especially for light nuclei [60].

Another route is to rely on microscopic calculations within a Hartree-Fock mean-field approximation for static properties and RPA for excitations, and to use the same model framework for calculation of the SNM. Such an approach allows a consistent determination of the breathing mode energy $E_{\mathrm{GMR}}$ and parameters of nuclear matter within the same framework. However, the microscopic approach has the disadvantage that the results are dependent on the choice of the effective interaction used in the models. The macroscopic approach is based only on the assumption that the liquid-drop model of the nucleus is valid and that the leptodermous expansion (23) converges reasonably fast.

The value of $K_{\mathrm{o}}$ most frequently used today is based on microscopic analysis of GMR data. Youngblood et al. [64] used measured $E 0$ strength distributions in ${ }^{40} \mathrm{Ca},{ }^{90} \mathrm{Zr}$, ${ }^{116} \mathrm{Sn},{ }^{144} \mathrm{Sm}$, and ${ }^{208} \mathrm{~Pb}$ and the Gogny interaction based on calculations by Blaizot et al. [60], which took into account pairing and anharmonicity in lighter nuclei. They deduced a value of $K_{\mathrm{o}}=231 \pm 5 \mathrm{MeV}$. This result was in good agreement with the value obtained by Farine et al. [65] of $K_{\mathrm{o}}=240 \mathrm{MeV}$ with generalized Skyrme forces. Myers and Świa̧tecki [66] used a model based on a semiclassical Thomas-Fermi approximation with a short-range Yukawa effective force between fermions. They obtained the value $K_{\mathrm{o}}=234 \mathrm{MeV}$ by fitting nuclear binding energies and diffuseness to experimental data. Later, Colò and co-workers [67] argued that there is no unique relationship between the value of $K_{\mathrm{o}}$ associated with an effective force and the GMR energy predicted by that force in nonrelativistic models. They built a new class of Skyrme forces and found that $230<K_{\mathrm{o}}<250 \mathrm{MeV}$. Agrawal et al. addressed the wellknown discrepancy between predictions of the value of $K_{\mathrm{o}}$ in nonrelativistic and relativistic models (see [68] and references therein) and concluded that the discrepancy, thought to be $\sim 20 \%$, can be much smaller for an appropriate choice of 
effective interactions. Todd-Rutel and Piekarewicz [69], using a new relativistic model FSUGold with two new parameters, causing softening both of the EoS of SNM and the density dependence of the symmetry energy, predicted $K_{\mathrm{o}}=230 \mathrm{MeV}$ in RMF calculations. However, new data on GMR in Sn isotopes [70,71] reopened the question of $K_{\mathrm{o}}$, as models which successfully predicted GMR energies in ${ }^{90} \mathrm{Zr},{ }^{144} \mathrm{Sm}$, and ${ }^{208} \mathrm{~Pb}$ could not reproduce GMR energies reported for ${ }^{112-124} \mathrm{Sn}$. We note that the new GMR energies for ${ }^{112,116,124} \mathrm{Sn}$ do not agree (just outside errors) with previous data [72,73]. It has been suggested that the new measurement by Li et al. offered cleaner spectra with less need for subtraction of background, which may possibly help to understand this discrepancy [71]. Colò et al. [74] tried to interpret the new data by employing a selfconsistent quasiparticle random-phase approximation model with Hartree-Fock-Bogoliubov basis, the Skyrme interaction, and density-dependent pairing. They reproduced the new GMR energies in ${ }^{112-120} \mathrm{Sn}$ isotopes using the SkM* Skyrme force and a surface pairing force and found that the effect of pairing on $K_{\mathrm{o}}$ is very small. However, the value of $K_{0}$ extracted from the fit to $\mathrm{Sn}$ isotopes is about $10 \%$ smaller than the one obtained from the fit to ${ }^{208} \mathrm{~Pb}, 230-240 \mathrm{MeV}$. A similar $\sim 10 \%$ discrepancy between $K_{0}$ from fits to $\mathrm{Sn}$ and $\mathrm{Pb}$ GMR energies has been reported in RMF calculations [75]. So, the puzzle of the GMR in Sn nuclei remains open [76]. Keeping in mind the current unresolved situation of GMR experiments and theory, and that all the extracted values are likely to be rather model dependent, we choose the following constraint for $K_{\mathrm{o}}$ (henceforth referred to as the SM1 constraint):

$$
\text { SM1: } K_{\mathrm{o}}=230 \pm 30 \mathrm{MeV} \text {. }
$$

To our knowledge, there has been only one attempt to constrain the third derivative of the energy per particle with respect to density, the skewness $K^{\prime}=-Q_{\text {o }}$ [see Eq. (8)], which is the next order fluctuation around the saturation density in the expansion [65]

$$
\mathcal{E}(\rho) / \rho \approx E_{\mathrm{o}}+\left(K_{\mathrm{o}} / 2\right) x^{2}-\left(K^{\prime} / 6\right) x^{3}+\cdots .
$$

Constraints on the skewness coefficient, which differ from author to author by a minus sign [65,77], are relatively imprecise. Farine et al. [65] tried to find acceptable values of $K^{\prime}$ by self-consistent analysis of breathing mode data using a selection of Skyrme forces. They found a subtle correlation between $K_{\mathrm{o}}$ and $K^{\prime}$. We adopt here their value of $K^{\prime}$ as constraint SM2,

$$
\text { SM2: } K^{\prime}=700 \pm 500 \mathrm{MeV} \text {. }
$$

The wide range of $K^{\prime}$ compensates for the fact that Farine $e t$ al. [65] used $K_{\mathrm{o}}=215 \pm 15 \mathrm{MeV}, 7 \%$ lower than our choice.

Limits on the pressure-density relationship in SNM and PNM and its curvature can be obtained from analysis of experimental data on the motion of ejected matter in energetic nucleus-nucleus collisions. Recently, measurements of the particle flow in collisions of ${ }^{197} \mathrm{Au}$ nuclei at incident kinetic energy per nucleon varying from about 0.15 to $10 \mathrm{GeV}$ were analyzed [78]. The authors extrapolated available data [79] for pressure at about $2 \rho_{\mathrm{o}}$ to higher densities, as well as to zero temperature. The results give limits on pressure as a function

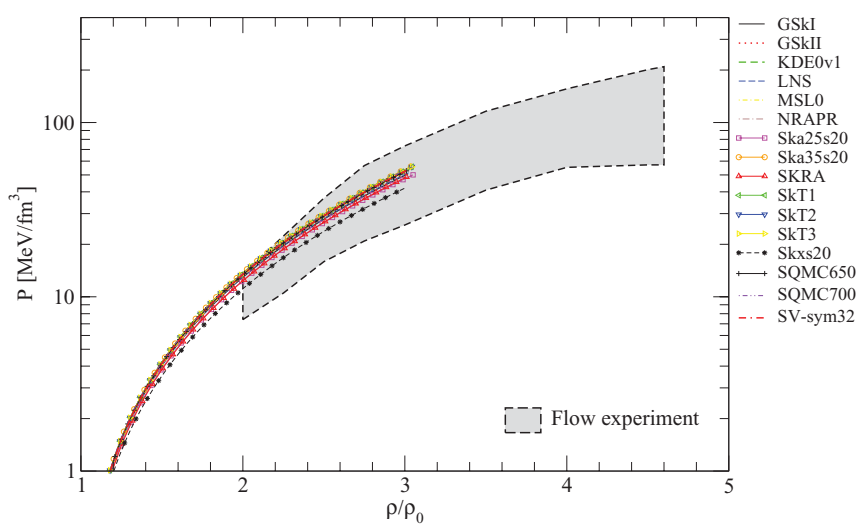

FIG. 1. (Color online) Constraint SM3: Pressure in SNM as a function of density up to $3 \frac{\rho}{\rho_{0}}$ as predicted by consistent Skyrme parametrizations. The shaded area in the region $2<\frac{\rho}{\rho_{0}}<4.6$ is taken from Ref. [78].

of density (see Fig. 1), which comprise the constraint SM3:

$$
\text { SM3: } P \text { vs } \frac{\rho}{\rho_{\mathrm{o}}}(y=0.5) \Longrightarrow \text { Flow Experiment. }
$$

Recent experimental investigation of kaon production in HIC produces a further constraint on pressure at a lower density region of $1.2 \leqslant \rho \leqslant 2.2 \mathrm{fm}^{-3}[80,81]$ (see Fig. 2), here named SM4:

SM4: $P$ vs $\frac{\rho}{\rho_{\mathrm{o}}}(y=0.5) \Longrightarrow$ Kaons + GMR Experiments.

\section{B. Pure neutron matter}

The EoS of PNM is of particular interest, because PNM is a realistic first approximation to the baryonic matter that comprises neutron stars. Most properties of neutron stars cannot be studied in terrestrial laboratories, and theoretical

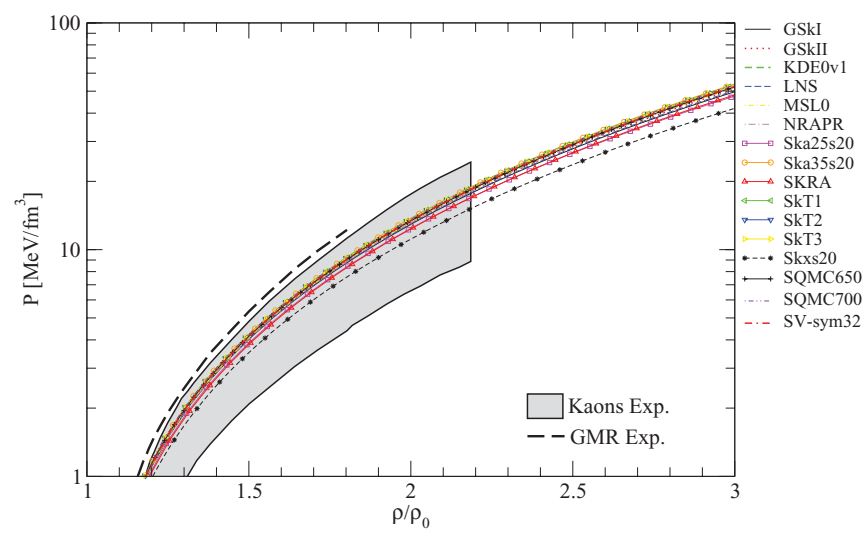

FIG. 2. (Color online) Constraint SM4: Pressure in symmetric nuclear matter as a function of density in the region $1.2<\frac{\rho}{\rho_{0}}<2.2$. The shaded area represents an educated guess discussed in Ref. [80] around the pressure-density relation available from kaon production data [81]. The dashed line extrapolates the pressures consistent with GMR data to higher densities in the region $1.2<\frac{\rho}{\rho_{0}}<1.7$. 
models, based on effective forces, must be used. However, at low densities, experiments with cold Fermi atoms yield information on strongly interaction fluids, very similar to the low-density neutron matter at neutron star crusts [41]. Different density regimes can be tuned by the magnitude of the neutron Fermi momentum $k_{\mathrm{F}}$ relative to the effective range $r_{\mathrm{o}}$ of the $N N$ interaction in the system [39]. The ground-state energy per particle, the EoS, can be expressed as

$$
\frac{E_{\mathrm{PNM}}}{E_{\mathrm{PNM}}^{o}}=\xi,
$$

where $E_{\mathrm{PNM}}$ is the energy per particle in Eq. (1) with $H_{\mathrm{n}}=2^{\mathrm{n}-1} \cdot E_{\mathrm{PNM}}=\mathcal{E}_{\mathrm{PNM}} / \rho$ and $E_{\mathrm{PNM}}^{\mathrm{o}}=3 \hbar^{2} k_{\mathrm{F}}^{2} / 10 M$, with $M$ being the mass of the nucleon. In the dilute degenerate Fermi gas regime, $k_{F} r_{\mathrm{o}} \ll 1, \xi$ is a constant [82]. This restricts the density below about $10^{-3} \rho_{\mathrm{o}}$, the density at which neutrons become unbound in neutron stars. At higher densities, below $\sim 0.1 \rho_{\mathrm{o}}$, where $k_{F} r_{\mathrm{o}} \approx 1, \xi$ has to be replaced by a system-dependent function $\xi\left(k_{\mathrm{F}}, r_{\mathrm{o}}\right)$. In this work we adopt the expression $E_{\mathrm{PNM}} / E_{\mathrm{PNM}}^{o}$ by Epelbaum et al. [40], based on next-to-leading order in lattice chiral effective field theory $\left(\mathrm{NLO}_{3}\right)$, and including corrections due to finite scattering length, nonzero effective range, and higher order corrections,

$$
\frac{E_{\mathrm{PNM}}}{E_{\mathrm{PNM}}^{o}}=\xi-\frac{\xi_{1}}{k_{F} a_{\mathrm{o}}}+c_{1} k_{F} r_{\mathrm{o}}+c_{2} k_{F}^{2} m_{\pi}^{-2}+c_{3} k_{F}^{3} m_{\pi}^{-3}+\cdots,
$$

where $m_{\pi}$ is the pion mass. The dimensionless universal constant $\xi$ has been determined from trapped cold atom experiments with ${ }^{6} \mathrm{Li}$ and ${ }^{40} \mathrm{~K}$, which yield a variety of values: $0.32_{-13}^{+10}$ [83], 0.51(4) [84], 0.46-05 [85], and 0.39(2) [86]. Values of $\xi_{1}$ in the literature are in the range $0.8-1.0$ ([40] and references therein). Epelbaum et al., using a simple Hamiltonian and only a few particles in their system, took $\xi=0.31$ and $\xi_{1}=0.81$ and fitted two sets of constants $\mathrm{c}_{1}, \mathrm{c}_{2}$, and $c_{3}$ : set $1(0.27,-0.44,0.0)$ and set $2(0.17,0.0,-0.26)$, and obtained a very similar quality fits to their $\mathrm{NLO}_{3}$. We

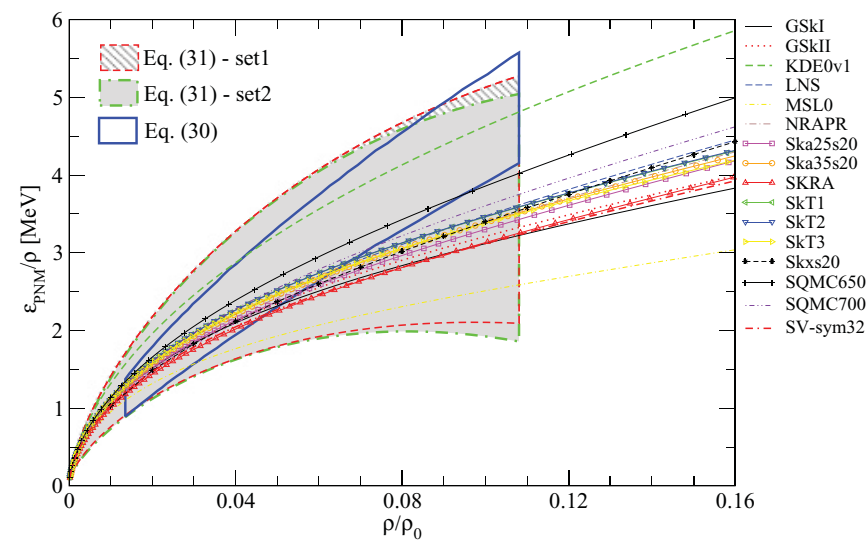

FIG. 3. (Color online) Constraint PNM1: Energy per particle in PNM as a function of density. The gray bands were based on Ref. [40] for two different parametrizations (dashed red and dashed-dotted green lines). The region inside the solid blue line illustrates the universal limit constraint given by Eq. (30) [39]. For more explanation see text. construct a constraint on energy per particle of PNM in the range of densities $0.01-0.1 \rho_{\mathrm{o}}$ shown in Fig. 3:

$$
\text { PNM1: } \frac{\mathcal{E}_{\mathrm{PNM}}}{\rho}(\mathrm{MeV}) \text { vs } \rho \text {. }
$$

The two shaded areas are based on Eq. (31) with $\xi_{1}=0.81$ and set 1 (red dashed line) or set 2 (green dashed-dotted line). The area inside the blue solid line is based on Eq. (30), the unitary limit. The boundaries of all three areas are calculated by taking $0.2<\xi<0.6$, which allows for the spread in experimental values.

It is clear that our PNM1 constraint is consistent with a relatively large range of extrapolated experimental $\xi$ values. Very recently, after our work was completed, more accurate calculations and measurements were reported. The new limits on $\xi$ are $0.37-0.38$ [87] and $0.38-0.41$ [88]. $\xi_{1}$, related to the contact parameter in unitary Fermi gases, has been calculated to be $\xi_{1}=0.9$ ([88] and references therein). These new data will be considered in future development of the PNM1 constraint.

New theoretical calculations also provide constraints on the EoS of low-density PNM. Gezerlis and Carlson ([42] and references therein) compare their EoS, obtained using quantum Monte Carlo techniques, with results of other model calculations in their Figs. 3 and 4. Although we still keep the
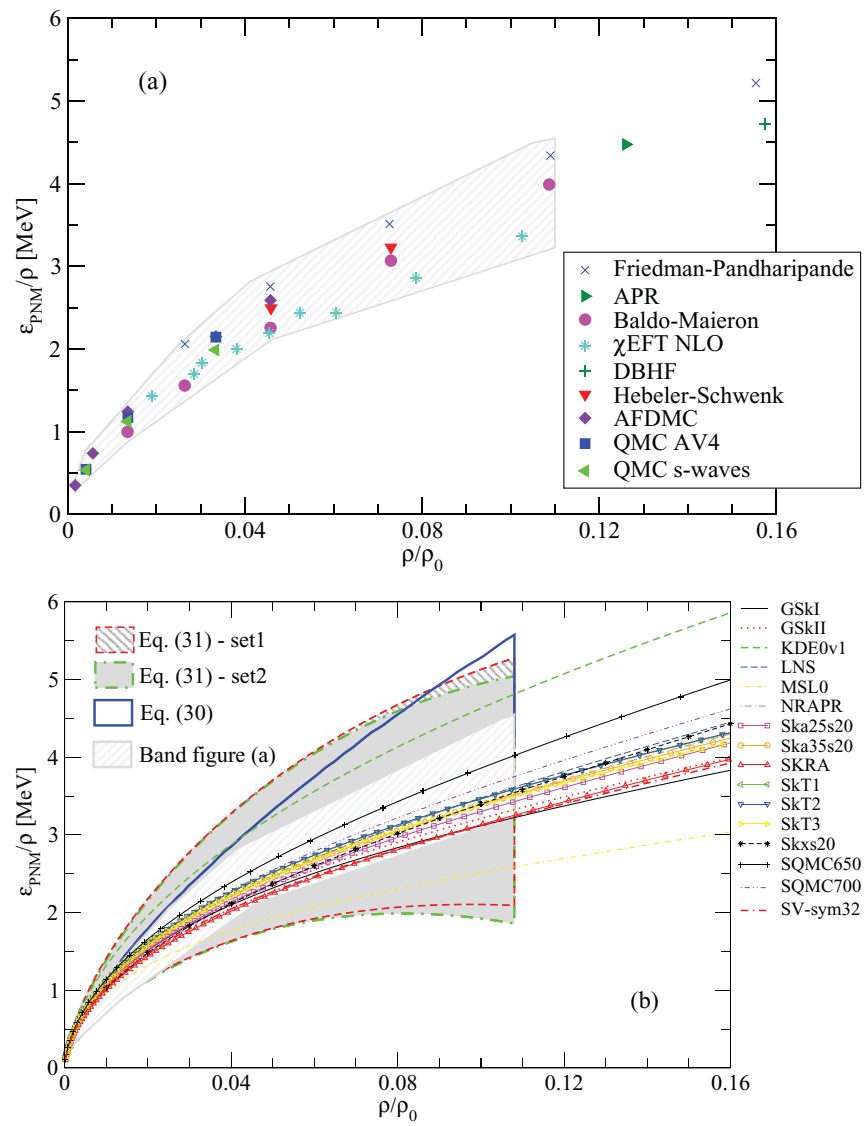

FIG. 4. (Color online) EoS of low-density pure neutron matter. (a) Band defined by results of theoretical calculations summarized in Ref. [42]. See the reference for explanation of the legend. (b) The same as in Fig. 3, but with the additional band (a) included. 


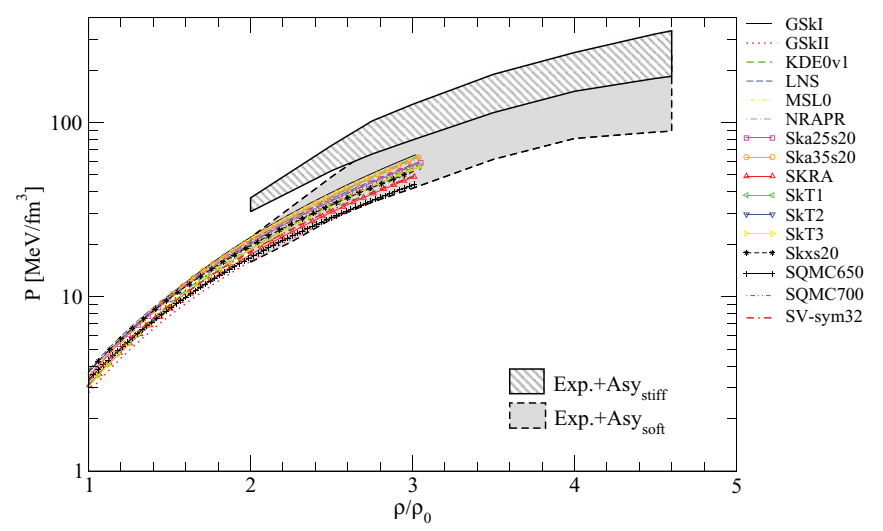

FIG. 5. (Color online) Constraint PNM2: Pressure in the PNM as a function of density as calculated by consistent Skyrme parametrizations up to $3 \frac{\rho}{\rho_{0}}$. The bands are in the region $2<\frac{\rho}{\rho_{0}}<4$.6. For detailed explanation see Ref. [110].

PNM1 as selector of the low-density neutron matter EoS in this paper, we have constructed a band, representing the boundaries of theoretical predictions, in the same $\rho / \rho_{\mathrm{o}}$ range used in the PNM1 constraint. This band is considerably narrower than the band extracted from the cold atom data. It is shown in Fig. 4(a) and merged with the PNM1 constraint in Fig. 4(b).

In the high-density region, analysis of HIC data [78] provides a constraint on the pressure-density relation in PNM:

$$
\text { PNM2: } P \text { vs } \frac{\rho}{\rho_{\mathrm{o}}}(y=0) \Longrightarrow \text { Flow Experiment. }
$$

The constraint is illustrated in Fig. 5.

\section{Constraints involving both SNM and PNM}

Nuclear symmetry energy, in particular its density dependence, has received considerable attention during the last decade. It produces information about the isospin dependence of nuclear forces which is equally important in nuclear matter and finite nuclei. The current empirical values of $J$, the symmetry energy at saturation density, as predicted by different models, vary around the value extracted from the upto-date finite-range liquid-droplet model $J=32.5 \mathrm{MeV}$ [89]. The data for $\mathcal{S}(\rho)$ come from several sources: HIC [80,90-92], pygmy dipole resonances (PDR) $[91,93,94]$, isobaric analog states (IAS) [95], and numerous microscopic calculations. A systematic difference exists between predictions of HF (Skyrme) and RMF models [44,96] spanning the range $27<$ $J<38 \mathrm{MeV}$. This large uncertainty stems, in part, from limited experimental knowledge and understanding of the isospin dependence of the nucleon-nucleon interaction and, in particular, the PNM EoS. Taking into account some more recent data [97], we define this constraint on $J$ as

$$
\text { MIX1: } 30<J<35 \mathrm{MeV} \text {. }
$$

The density dependence of the symmetry energy, especially at super-nuclear densities, has direct relevance for modeling neutron stars $[96,98]$ and is closely related to studies of neutron matter radii and the neutron skin in neutron-heavy nuclei $[99,100]$. In contrast to the expansion of the energy per particle, in which the term containing the first derivative vanishes, the expansion of $\mathcal{S}(\rho)$, Eq. (13), contains a first-order correction $L$ at $\rho=\rho_{\mathrm{o}}$. $L$ becomes an important bulk quantity that determines most of the behavior of $\mathcal{S}(\rho)$ in the vicinity of $\rho_{\mathrm{o}}$. The empirical determination of $L$ is, as for several other bulk quantities, indirect. The very recent constraint, based on the empirical modified Skyrme-like (MSL) model with the MSL0 Skyrme-like interaction, is based on analysis of isospin diffusion and the double neutron/proton ratio in heavy-ion collisions at intermediate energies, and it requires [101]

$$
\text { MIX2: } L=58 \pm 18 \mathrm{MeV} \text {. }
$$

We comment that this value is lower than the previously accepted $L=88 \pm 25 \mathrm{MeV}$ [102] derived by the same group but considering only the isospin diffusion data and standard Skyrme forces. This lower value of $L$ is supported by findings of Newton and Li [103], who used the correlation between the gravitational binding energy of the low-mass neutron star PSR J0737-3039B and the slope of the nuclear symmetry energy at 1-2 times the nuclear saturation density. This correlation leads to an upper limit of $L \leqslant 70 \mathrm{MeV}$. It is also consistent with the value $L=49.9 \mathrm{MeV}$ determined from the droplet model [104] and closer to the most recent value from the finite-range droplet model (FRDM) of $L=70 \pm 15 \mathrm{MeV}$ [89]. Vidaña et al. [57] found a value of $L=66.5 \mathrm{MeV}$ in their Bruckner-Hartree-Fock calculation with the Argonne V18 potential supplemented by a three-body force of Urbana type. As $J$ and $L$ are correlated, some investigations produce a range of acceptable values for both of these observables (see, e.g., $[93,97]$ for a recent summary).

The next constraint involves the isospin incompressibility $K_{\tau}$ in Eq. (23) for the compression modulus of a finite nucleus with mass $A$. This constraint on $K_{\tau}$, which is only dependent on the validity of the expansion (36), from experiment was provided by Li et al. [70,71,105], who measured GMR strength distributions for ${ }^{112-124} \mathrm{Sn}$ and ${ }^{106,110-116} \mathrm{Cd}$ isotopes in inelastic scattering of $\alpha$ particles. They used a simplified expression for the compression modulus $K_{A}$ [as compared to Eq. (23)],

$$
K_{\mathrm{A}}=K_{\mathrm{vol}}+K_{\text {surf }} A^{-1 / 3}+K_{\tau} \beta^{2}+K_{\mathrm{Coul}} \frac{Z^{2}}{A^{4 / 3}},
$$

omitting the higher order terms in $A$ and $\beta . \mathrm{Li}$ et al. further assumed that $K_{\text {surf }}=-K_{\text {vol }}$ and $K_{\text {vol }}=K_{\mathrm{o}}$ (the scaling approximation). $K_{\text {Coul }}$ was taken to be $-5.2 \pm 0.7 \mathrm{MeV}$. This value has been derived by Sagawa et al. [106], who investigated the microscopic structure of $K_{\text {Coul }}$ and its correlation with $K_{\mathrm{o}}$, using a set of thirteen Skyrme parametrizations and seven RMF Lagrangians. They found this correlation to be rather weak and arrived at an average value of $K_{\mathrm{Coul}}$, used by Li et al. This procedure yielded $K_{\tau}=-550 \pm 100 \mathrm{MeV}$.

It is important to realize that the $K_{\tau}$ value, extracted by $\mathrm{Li}$ et al., includes both the volume and the surface components of the isospin compressibility $K_{\tau}$, Eq. (24). It follows that, in order to compare the microscopic model calculation with the constraint, the contribution of the surface-symmetry term, which is difficult to calculate exactly, must be at least estimated as well as possible and subtracted from $K_{\tau}$. Stone et al. [107] reanalyzed the combined $\mathrm{Sn}+\mathrm{Cd}$ data by Li et al. and Garg under the same conditions and found $K_{\tau}=-595 \pm 154 \mathrm{MeV}$. 
Estimation of $K_{\tau, \mathrm{v}}$ and $K_{\tau, \mathrm{s}}$ from the currently available GMR data is not easy. The data show systematic differences, mainly dependent on methods used for analysis by different groups. It is therefore necessary to include some additional constraints on the fit to obtain limits on $K_{\tau, \mathrm{v}}$ and $K_{\tau, \mathrm{s}}$. Stone et al. used two assumptions. First, they required that Eq. (24) holds and looked for all combinations of $K_{\tau, \mathrm{v}}$ and $K_{\tau, \mathrm{s}}$ which would satisfy it. $K_{\tau, \mathrm{v}}$ is expected to be negative, in line with microscopic calculations. It was varied in the region of $-1200<K_{\tau, \mathrm{v}}<$ $0 \mathrm{MeV}$ with $K_{\tau, \mathrm{s}}$ in the range of $-1600<K_{\tau, \mathrm{s}}<1600 \mathrm{MeV}$. The second assumption was that the expansion (24) in terms of $A^{-1 / 3}$ and $\beta^{2}$ converges at a reasonable rate, i.e., no higher order terms are significant. They took

$$
\frac{K_{\tau, s} A^{-1 / 3}}{K_{\tau, v}} \leqslant 0.5 .
$$

Simultaneous application of Eqs. (24) and (37), together with the assumption that $K_{\tau, v}$ is negative, allows limits on $K_{\tau, v}$ to be extracted and taken as a constraint,

$$
\text { MIX3: }-760 \leqslant K_{\tau, v} \leqslant-372 \mathrm{MeV} .
$$

Corresponding limits on the surface contribution to isospin incompressibility are $-1110 \leqslant K_{\tau, s} \leqslant 960 \mathrm{MeV}$. We comment that the condition on the ratio of the contribution of the surface and volume isospin incompressibility is rather conservative. Treiner et al. [62] estimated the surface contribution to the isospin incompressibility to be $\sim 20 \%$ of the volume term in ${ }^{208} \mathrm{~Pb}$ in the scaling approximation, implying a rapid convergence of expansion, Eq. (23). We note that other suggested limits on $K_{\tau, \mathrm{v}}=-370 \pm 120 \mathrm{MeV}$ (in the notation of the original paper [108] $K_{\tau, 2}^{\text {sat }}$ ) exist in the literature, but they are calculated, not directly extracted from experimental data. Patra et al. [109] estimated $K_{\tau, \mathrm{v}}$ and $K_{\tau, s}$ using a semiclassical relativistic mean-field method with NL1, NL3, and NLSH interactions. They obtained $K_{\tau, \mathrm{v}}=-676,-690$, and $-794 \mathrm{MeV}$ and $K_{\tau, \mathrm{v}}=1951,1754$, and $1716 \mathrm{MeV}$ for the three interactions, NL1, NL3, and NLSH, respectively.

As the next constraint, we used the known effect that, at the nuclear surface, variation of the difference between proton and neutron densities (neutron skin) is expected. Danielewicz [110] considered this question and proposed limits to the reduction of the symmetry energy at $\rho_{\mathrm{o}} / 2$ in terms of $\mathcal{S}\left(\rho_{\mathrm{o}} / 2\right) / J$. These limits, based on a semiclassical Thomas-Fermi model and a comparison of the neutron skin of ${ }^{208} \mathrm{~Pb}$ as calculated in this model with values from full mean-field models, lead to

$$
\text { MIX4: } 0.57<\frac{\mathcal{S}\left(\rho_{\mathrm{o}} / 2\right)}{J}<0.83 .
$$

Finally, Piekarewicz $[111,112]$ used a parabolic approximation to the EoS and derived an expression for the pressure in pure neutron matter, related to the slope of the symmetry energy $L$ at saturation density. Thus the "symmetry pressure" $L$, a quantity that influences the neutron-skin thickness in heavy nuclei, is directly proportional to the pressure of pure neutron matter as $3 P_{\mathrm{PNM}}\left(\rho_{\mathrm{o}}\right) /\left(L \rho_{\mathrm{o}}\right) \approx 1$ [112]. Considering the uncertainty in the number of terms included in the expansion, we introduce a range to this constraint as

$$
\text { MIX5: } \frac{3 P_{\mathrm{PNM}}\left(\rho_{\mathrm{o}}\right)}{L \rho_{\mathrm{o}}}=1 \pm 0.1 \text {. }
$$

In terms of Skyrme model parameters, this constraint can be expressed analytically as

$$
\frac{3 P_{\mathrm{PNM}}\left(\rho_{\mathrm{o}}\right)}{L \rho_{\mathrm{o}}}=1+\frac{1}{L} \sum_{i=1}^{6} T_{i},
$$

where

$$
\begin{aligned}
T_{1}= & \left(\frac{9 \sqrt[3]{4}}{5}-1\right) \frac{\hbar^{2}}{3 M}\left(\frac{3 \pi^{2}}{2}\right)^{2 / 3} \rho_{\mathrm{o}}^{2 / 3}, \\
T_{2}= & \frac{9 t_{0}}{8} \rho_{\mathrm{o}}, \quad T_{3}=\frac{3}{16} \sum_{i=1}^{3} t_{3 i}\left(\sigma_{i}+1\right) \rho^{\sigma_{i}+1}, \\
T_{4}= & \frac{5}{24}\left(\frac{3 \pi^{2}}{2}\right)^{2 / 3}\left[a\left(\frac{9 \sqrt[3]{4}}{5}-1\right)+2 b\left(\frac{9 \sqrt[3]{4}}{5}-2\right)\right] \rho_{\mathrm{o}}^{5 / 3}, \\
T_{5}= & \frac{1}{8}\left(\frac{3 \pi^{2}}{2}\right)^{2 / 3}(5+3 \delta)\left[\frac{3 \sqrt[3]{4}}{5}\left(1-x_{4}\right)+x_{4}\right] t_{4} \rho_{\mathrm{o}}^{\frac{5}{3}+\delta} \\
T_{6}= & \frac{1}{8}\left(\frac{3 \pi^{2}}{2}\right)^{2 / 3}(5+3 \gamma)\left[\frac{9 \sqrt[3]{4}}{5}\left(1+x_{5}\right)-\frac{1}{3}\left(5 x_{5}+4\right)\right] \\
& \times t_{5} \rho_{\mathrm{o}}^{\frac{5}{3}+\gamma} .
\end{aligned}
$$

\section{Results of application of the macroscopic constraints}

The predictions of bulk properties of nuclear matter by all Skyrme parameter sets are summarized in Table II. Table III details whether a particular parameter set is consistent $(+)$ or not $(-)$ with each of the eleven constraints.

Numerical evaluation of the compliance for individual parametrizations with the constraints is given in Table IV. The criterion for consistency with a constraint is different for numerical (SM1, SM2, and MIX1-5) and graphical (SM3, SM4, PNM1, and PNM2) constraints. For the numerical ones, we define a deviation

$$
\operatorname{Dev}=\frac{Q_{\text {mod }}-Q_{\text {const }}}{\Delta},
$$

where $Q_{\text {mod }}$ is the value of a specific quantity calculated in the model, and $Q_{\text {const }}$ is the central value of the related constraint. The error associated with $Q_{\text {const }}$ is $\Delta$. If $|\mathrm{Dev}| \leqslant 1$, the model is consistent. In particular, for the MIX1, MIX3, and MIX4 constraints that are defined by a range in the form $x_{1} \leqslant X \leqslant$ $x_{2}$, we define the central value as $Q_{\text {const }}=\left(x_{2}+x_{1}\right) / 2$ and the error as $\Delta=x_{2}-Q_{\text {const }}=Q_{\text {const }}-x_{1}$.

For the graphic constraints SM3, SM4, PNM1, and PNM2, we consider as consistent a model that is inside of the corresponding band in $95 \%$ or more of the density region. In this case, the Skyrme interaction is rendered valid.

Table V shows the number of parameter sets (out of 240) which satisfied each constraint. This table shows that no individual constraint is particularly discriminative. It is more interesting to consider further the parameter sets which satisfy all constraints. They are surprisingly few in number-only sets LNS, NRAPR, Ska25s20, SQMC650, SQMC700, and SV-sym32 are selected. 
TABLE II. Saturation properties of all Skyrme parametrizations used in this work. All entries are in MeV, except for the saturation density $\rho_{\mathrm{o}}$ in $\mathrm{fm}^{-3}$ and the dimensionless effective mass $m^{*}=M^{*} / M$.

\begin{tabular}{|c|c|c|c|c|c|c|c|c|c|c|}
\hline BSk1 [160] & 0.157 & -15.81 & 231.31 & 385.59 & 27.81 & 7.19 & -281.83 & 606.46 & -312.97 & 1.05 \\
\hline BSk2 [161] & 0.157 & -15.80 & 233.65 & 380.07 & 28.00 & 7.98 & -296.98 & 557.91 & -331.87 & 1.04 \\
\hline BSk3 [162] & 0.157 & -15.81 & 234.81 & 380.83 & 27.93 & 6.78 & -306.90 & 550.34 & -336.59 & 1.12 \\
\hline BSk4 [163] & 0.157 & -15.77 & 236.84 & 367.17 & 28.00 & 12.54 & -265.93 & 558.40 & -321.74 & 0.92 \\
\hline BSk5 [163] & 0.157 & -15.80 & 237.19 & 367.86 & 28.70 & 21.41 & -240.30 & 499.92 & -335.56 & 0.92 \\
\hline BSk7 [163] & 0.157 & -15.76 & 229.26 & 370.92 & 28.00 & 17.99 & -209.35 & 598.16 & -288.18 & 0.80 \\
\hline BSk8 [164] & 0.159 & -15.83 & 230.31 & 372.39 & 28.00 & 14.85 & -220.88 & 624.89 & -285.98 & 0.80 \\
\hline BSk9 [165] & 0.159 & -15.92 & 231.32 & 374.67 & 30.00 & 38.29 & -153.70 & 482.61 & -321.44 & 0.80 \\
\hline BSk10 [166] & 0.159 & -15.91 & 238.83 & 370.34 & 30.00 & 37.24 & -194.90 & 396.99 & -360.60 & 0.92 \\
\hline BSk11 [166] & 0.159 & -15.86 & 238.09 & 369.18 & 30.00 & 38.36 & -189.81 & 390.14 & -360.48 & 0.92 \\
\hline BSk12 [166] & 0.159 & -15.86 & 238.06 & 369.11 & 30.00 & 38.01 & -191.35 & 392.53 & -360.47 & 0.92 \\
\hline BSk17 [170] & 0.159 & -16.06 & 241.69 & 363.62 & 30.00 & 36.29 & -181.84 & 450.48 & -344.97 & 0.80 \\
\hline BSk18 [52] & 0.159 & -16.06 & 241.79 & 363.82 & 30.00 & 36.22 & -180.90 & 454.52 & -343.71 & 0.80 \\
\hline BSk19 [130] & 0.160 & -16.08 & 237.33 & 297.89 & 30.00 & 31.90 & -191.44 & 472.94 & -342.79 & 0.80 \\
\hline BSk20 [130] & 0.160 & -16.08 & 241.39 & 282.26 & 30.00 & 37.38 & -136.49 & 549.73 & -317.05 & 0.80 \\
\hline BSk21 [130] & 0.158 & -16.05 & 245.80 & 274.09 & 30.00 & 46.56 & -37.20 & 709.66 & -264.62 & 0.80 \\
\hline E [171] & 0.159 & -16.13 & 333.46 & 63.72 & 27.66 & -31.27 & -570.73 & 448.61 & -389.09 & 0.87 \\
\hline Es [171] & 0.163 & -16.02 & 248.60 & 352.41 & 26.44 & -36.86 & -457.76 & 880.01 & -288.86 & 0.84 \\
\hline$f_{-}[153]$ & 0.162 & -16.02 & 230.01 & 404.93 & 32.00 & 43.78 & -105.08 & 654.90 & -290.70 & 0.70 \\
\hline$f_{+}[153]$ & 0.162 & -16.04 & 230.01 & 406.17 & 32.00 & 41.54 & -117.98 & 661.07 & -293.85 & 0.70 \\
\hline$f_{0}[153]$ & 0.162 & -16.03 & 230.01 & 405.45 & 32.00 & 42.41 & -113.41 & 657.36 & -293.11 & 0.70 \\
\hline FPLyon [172] & 0.162 & -15.92 & 217.03 & 399.45 & 30.93 & 42.76 & -135.60 & 485.79 & -313.47 & 0.84 \\
\hline GSkI [51] & 0.159 & -16.02 & 230.21 & 405.58 & 32.03 & 63.45 & -95.29 & 293.44 & -364.19 & 0.78 \\
\hline GSkII [51] & 0.159 & -16.12 & 233.40 & 398.73 & 30.49 & 48.63 & -157.83 & 310.27 & -366.54 & 0.79 \\
\hline KDE [173] & 0.164 & -15.99 & 223.90 & 381.81 & 31.97 & 41.42 & -141.83 & 543.33 & -319.71 & 0.76 \\
\hline KDE0v [173] & 0.161 & -16.10 & 228.71 & 373.39 & 32.98 & 45.21 & -144.78 & 523.27 & -342.24 & 0.72 \\
\hline KDE0v1 [173] & 0.165 & -16.23 & 227.54 & 384.86 & 34.58 & 54.69 & -127.12 & 484.45 & -362.78 & 0.74 \\
\hline LNS [118] & 0.175 & -15.32 & 210.78 & 382.55 & 33.43 & 61.45 & -127.36 & 302.46 & -384.55 & 0.83 \\
\hline MSk1 [174] & 0.157 & -15.83 & 233.73 & 379.97 & 30.00 & 33.92 & -200.02 & 448.66 & -348.39 & 1.00 \\
\hline MSk2 [174] & 0.157 & -15.83 & 231.65 & 386.21 & 30.00 & 33.35 & -203.44 & 449.71 & -347.94 & 1.05 \\
\hline MSk3 [174] & 0.157 & -15.79 & 233.25 & 379.01 & 28.00 & 7.04 & -283.52 & 615.65 & -314.33 & 1.00 \\
\hline MSk4 [174] & 0.157 & -15.79 & 231.17 & 385.26 & 28.00 & 7.20 & -284.05 & 610.93 & -315.24 & 1.05 \\
\hline MSk5 [174] & 0.157 & -15.79 & 231.17 & 385.26 & 28.00 & 7.57 & -282.55 & 607.93 & -315.36 & 1.05 \\
\hline $\mathrm{MSk}^{*}$ [119] & 0.156 & -15.78 & 243.74 & 346.15 & 28.00 & 7.02 & -290.66 & 595.12 & -322.81 & 0.80 \\
\hline MSk6 [174] & 0.157 & -15.79 & 231.17 & 385.26 & 28.00 & 9.63 & -274.33 & 591.49 & -316.05 & 1.05 \\
\hline MSk7 [175] & 0.157 & -15.80 & 231.22 & 385.36 & 27.95 & 9.40 & -274.63 & 592.08 & -315.38 & 1.05 \\
\hline MSk8 [175] & 0.157 & -15.80 & 229.31 & 391.01 & 27.93 & 8.26 & -280.01 & 597.59 & -315.49 & 1.10 \\
\hline MSk9 [175] & 0.157 & -15.80 & 233.33 & 379.16 & 28.00 & 10.36 & -270.23 & 589.06 & -315.57 & 1.00 \\
\hline MSkA [176] & 0.153 & -15.99 & 313.33 & 138.15 & 30.35 & 57.17 & -135.34 & 197.74 & -453.13 & 0.79 \\
\hline MSL0 [101] & 0.160 & -16.00 & 230.00 & 380.32 & 30.00 & 60.00 & -99.33 & 224.29 & -360.11 & 0.80 \\
\hline NRAPR [177] & 0.161 & -15.85 & 225.65 & 362.54 & 32.78 & 59.63 & -123.32 & 311.61 & -385.32 & 0.69 \\
\hline PRC45 [178] & 0.145 & -15.82 & 367.58 & -165.69 & 51.01 & 141.52 & -23.01 & 92.05 & -935.89 & 1.00 \\
\hline
\end{tabular}


TABLE II. (Continued.)

\begin{tabular}{|c|c|c|c|c|c|c|c|c|c|c|}
\hline RATP [179] & 0.160 & -16.05 & 239.52 & 349.83 & 29.26 & 32.39 & -191.23 & 440.70 & -338.28 & 0.67 \\
\hline Rs [171] & 0.158 & -15.59 & 237.42 & 348.46 & 30.82 & 86.39 & -9.21 & 22.41 & -400.74 & 0.78 \\
\hline Sefm074 [180] & 0.160 & -15.81 & 240.10 & 350.15 & 33.40 & 88.73 & -33.14 & 58.41 & -436.12 & 0.74 \\
\hline Sefm081 [180] & 0.161 & -15.69 & 237.04 & 356.66 & 30.76 & 79.39 & -39.54 & 66.74 & -396.41 & 0.81 \\
\hline SGI [181] & 0.154 & -15.89 & 261.75 & 297.93 & 28.33 & 63.86 & -51.99 & 194.46 & -362.49 & 0.61 \\
\hline SGII [181] & 0.158 & -15.60 & 214.65 & 380.91 & 26.83 & 37.63 & -145.90 & 330.41 & -304.90 & 0.79 \\
\hline SGOI [182] & 0.168 & -16.63 & 361.59 & -37.36 & 45.20 & 99.76 & -155.64 & 144.36 & -764.53 & 0.61 \\
\hline SGOII [182] & 0.168 & -16.70 & 253.28 & 346.18 & 93.98 & 246.02 & -119.57 & 272.39 & -1259.44 & 0.61 \\
\hline SI [27] & 0.155 & -15.99 & 370.38 & -152.32 & 29.24 & 1.22 & -461.84 & 141.44 & -469.66 & 0.91 \\
\hline SII [27] & 0.148 & -15.99 & 341.40 & -15.76 & 34.16 & 50.02 & -265.72 & 104.75 & -568.17 & 0.58 \\
\hline SK255 [68] & 0.157 & -16.33 & 254.93 & 350.09 & 37.40 & 95.05 & -58.33 & 94.23 & -498.11 & 0.80 \\
\hline SK272 [68] & 0.155 & -16.28 & 271.51 & 305.31 & 37.40 & 91.67 & -67.78 & 134.36 & -514.70 & 0.77 \\
\hline SkA [186] & 0.155 & -15.99 & 263.16 & 300.13 & 32.91 & 74.62 & -78.46 & 174.54 & -441.08 & 0.61 \\
\hline Ska25s20 [187] & 0.161 & -16.07 & 220.75 & 413.45 & 33.78 & 63.81 & -118.22 & 314.13 & -381.56 & 0.98 \\
\hline Ska35s15 [187] & 0.158 & -16.01 & 238.89 & 378.88 & 30.56 & 30.60 & -222.90 & 481.99 & -357.96 & 1.01 \\
\hline Ska35s20 [187] & 0.158 & -16.08 & 240.27 & 378.65 & 33.57 & 64.83 & -120.32 & 284.54 & -407.11 & 1.00 \\
\hline Ska35s25 [187] & 0.158 & -16.14 & 241.30 & 378.94 & 36.98 & 98.89 & -23.57 & 97.46 & -461.60 & 0.99 \\
\hline Ska45s20 [187] & 0.156 & -16.08 & 260.21 & 330.55 & 33.39 & 66.21 & -119.99 & 251.77 & -433.13 & 1.02 \\
\hline SkB [186] & 0.155 & -15.99 & 263.16 & 300.13 & 23.88 & 47.54 & -78.46 & 174.54 & -309.50 & 0.61 \\
\hline SkI1 [188] & 0.160 & -15.95 & 242.75 & 346.14 & 37.53 & 161.05 & 234.67 & -328.02 & -502.01 & 0.69 \\
\hline SkI2 [188] & 0.158 & -15.78 & 240.93 & 339.70 & 33.37 & 104.33 & 70.69 & 51.62 & -408.21 & 0.68 \\
\hline SkMP [192] & 0.157 & -15.56 & 230.87 & 338.05 & 29.89 & 70.31 & -49.82 & 159.44 & -368.73 & 0.65 \\
\hline SkO [193] & 0.160 & -15.84 & 223.34 & 392.86 & 31.97 & 79.14 & -43.17 & 131.13 & -378.80 & 0.90 \\
\hline $\mathrm{SkO}^{\prime}$ [193] & 0.160 & -15.75 & 222.36 & 390.83 & 31.95 & 68.94 & -78.82 & 223.37 & -371.29 & 0.90 \\
\hline SkP [194] & 0.163 & -15.95 & 200.97 & 435.43 & 30.00 & 19.68 & -266.60 & 508.35 & -342.04 & 1.00 \\
\hline SKRA [195] & 0.159 & -15.78 & 216.98 & 378.76 & 31.32 & 53.04 & -139.28 & 310.84 & -364.92 & 0.75 \\
\hline SkS1 [196] & 0.161 & -15.86 & 228.43 & 382.76 & 28.75 & 30.52 & -218.69 & 379.24 & -350.66 & 0.86 \\
\hline SkS2 [196] & 0.161 & -15.89 & 229.02 & 382.73 & 29.23 & 37.84 & -218.07 & 270.03 & -381.86 & 0.85 \\
\hline SkS3 [196] & 0.161 & -15.88 & 228.83 & 382.62 & 28.84 & 51.74 & -157.38 & 154.06 & -381.30 & 0.85 \\
\hline SkS4 [196] & 0.163 & -15.88 & 228.08 & 385.45 & 28.35 & 23.28 & -238.42 & 438.06 & -338.77 & 0.87 \\
\hline SkSC1 [197] & 0.161 & -15.85 & 234.58 & 380.50 & 28.10 & 0.13 & -312.03 & 673.32 & -312.62 & 1.00 \\
\hline SkSC2 [197] & 0.161 & -15.90 & 235.13 & 381.60 & 24.74 & 11.00 & -228.22 & 505.69 & -276.35 & 1.00 \\
\hline SkSC3 [197] & 0.161 & -15.85 & 234.49 & 380.32 & 27.01 & 0.81 & -296.20 & 641.65 & -299.75 & 1.00 \\
\hline SkSC4 [198] & 0.161 & -15.87 & 234.72 & 380.79 & 28.80 & -2.12 & -329.49 & 708.23 & -320.20 & 1.00 \\
\hline SkSC4o [199] & 0.161 & -15.87 & 234.74 & 380.79 & 27.00 & -9.67 & -338.03 & 725.33 & -295.70 & 1.00 \\
\hline SkSC5 [200] & 0.161 & -15.85 & 234.50 & 380.34 & 30.99 & -6.97 & -375.08 & 799.41 & -344.58 & 1.00 \\
\hline SkSC6 [200] & 0.161 & -15.92 & 235.41 & 382.13 & 24.57 & 11.00 & -226.26 & 501.80 & -274.39 & 1.00 \\
\hline SkSC10 [200] & 0.161 & -15.96 & 235.89 & 383.08 & 22.83 & 19.13 & -172.77 & 394.81 & -256.47 & 1.00 \\
\hline SkSC11 [201] & 0.161 & -15.87 & 234.72 & 380.79 & 28.80 & -2.12 & -329.49 & 708.23 & -320.20 & 1.00 \\
\hline SkSC14 [199] & 0.161 & -15.92 & 235.41 & 382.13 & 30.00 & 33.13 & -202.83 & 454.93 & -347.84 & 1.00 \\
\hline SkSC15 [199] & 0.161 & -15.88 & 234.93 & 381.17 & 28.00 & 6.72 & -284.47 & 618.21 & -313.89 & 1.00 \\
\hline SkSP.1 [119] & 0.162 & -15.90 & 230.02 & 502.64 & 28.00 & 7.17 & -289.55 & 662.66 & -316.92 & 0.80 \\
\hline SkT [202] & 0.148 & -15.40 & 333.36 & -29.01 & 33.66 & 80.83 & -78.93 & 69.87 & -570.95 & 0.60 \\
\hline SkT1 [113] & 0.161 & -15.98 & 236.16 & 383.52 & 32.02 & 56.18 & -134.83 & 318.99 & -380.68 & 1.00 \\
\hline
\end{tabular}


TABLE II. (Continued.)

\begin{tabular}{|c|c|c|c|c|c|c|c|c|c|c|}
\hline SkT2 [113] & 0.161 & -15.94 & 235.73 & 382.67 & 32.00 & 56.16 & -134.67 & 318.66 & -380.48 & 1.00 \\
\hline SkT3 [113] & 0.161 & -15.95 & 235.74 & 382.70 & 31.50 & 55.31 & -132.05 & 313.43 & -374.14 & 1.00 \\
\hline SkT5 [113] & 0.164 & -16.00 & 201.69 & 436.81 & 37.00 & 98.53 & -24.97 & 99.88 & -402.76 & 1.00 \\
\hline SkT6 [113] & 0.161 & -15.96 & 235.95 & 383.15 & 29.97 & 30.85 & -211.53 & 472.36 & -346.54 & 1.00 \\
\hline SkT9 [113] & 0.160 & -15.88 & 234.91 & 370.97 & 29.76 & 33.74 & -185.62 & 471.98 & -334.76 & 0.83 \\
\hline $\mathrm{SkT}^{*}{ }^{*}[113]$ & 0.162 & -16.20 & 238.95 & 388.75 & 32.31 & 56.58 & -136.66 & 322.86 & -384.07 & 1.00 \\
\hline SkT3* [113] & 0.162 & -16.20 & 238.95 & 388.76 & 31.97 & 56.32 & -133.65 & 316.82 & -379.93 & 1.00 \\
\hline SkT1a [180] & 0.161 & -15.98 & 236.16 & 383.52 & 32.02 & 56.18 & -134.83 & 318.99 & -380.68 & 1.00 \\
\hline SkT2a [180] & 0.161 & -15.94 & 235.73 & 382.67 & 32.00 & 56.16 & -134.67 & 318.66 & -380.48 & 1.00 \\
\hline SkT3a [180] & 0.161 & -15.95 & 235.74 & 382.70 & 31.50 & 55.31 & -132.05 & 313.43 & -374.14 & 1.00 \\
\hline SkT8a [180] & 0.161 & -15.94 & 235.70 & 372.37 & 29.92 & 33.72 & -187.52 & 476.25 & -336.59 & 0.83 \\
\hline SkT9a [180] & 0.160 & -15.88 & 234.91 & 370.97 & 29.76 & 33.74 & -185.62 & 471.98 & -334.76 & 0.83 \\
\hline SkTK [203] & 0.168 & -16.70 & 253.28 & 346.18 & 35.57 & 41.59 & -221.79 & 527.94 & -414.46 & 0.61 \\
\hline SKX [204] & 0.155 & -16.05 & 271.06 & 297.42 & 31.10 & 33.18 & -252.12 & 379.69 & -414.81 & 0.99 \\
\hline SKXce [204] & 0.155 & -15.86 & 268.19 & 294.59 & 30.15 & 33.48 & -238.39 & 356.93 & -402.51 & 1.01 \\
\hline SKXm [204] & 0.159 & -16.04 & 238.09 & 380.38 & 31.20 & 32.08 & -242.76 & 428.73 & -384.00 & 0.97 \\
\hline Skxs15 [205] & 0.161 & -15.76 & 201.10 & 424.57 & 31.88 & 34.79 & -197.10 & 516.30 & -332.38 & 0.97 \\
\hline Skxs20 [205] & 0.162 & -15.81 & 201.95 & 425.56 & 35.50 & 67.06 & -122.31 & 328.52 & -383.37 & 0.96 \\
\hline Skxs25 [205] & 0.161 & -15.87 & 202.92 & -440.88 & 39.60 & 100.10 & -50.28 & 145.99 & -440.88 & 0.96 \\
\hline Skz-1 [128] & 0.160 & -16.01 & 230.08 & 365.25 & 32.00 & 54.14 & -184.08 & 217.03 & -422.99 & 0.70 \\
\hline Skz0 [128] & 0.160 & -16.01 & 230.08 & 365.24 & 32.00 & 35.10 & -242.20 & 405.16 & -397.08 & 0.70 \\
\hline SLy230a [45] & 0.160 & -15.99 & 229.89 & 364.18 & 31.99 & 44.32 & -98.22 & 602.87 & -293.91 & 0.70 \\
\hline SLy230b [45] & 0.160 & -15.97 & 229.91 & 363.10 & 32.01 & 45.97 & -119.72 & 521.50 & -322.92 & 0.69 \\
\hline SLy3 [206] & 0.160 & -15.94 & 229.51 & 362.56 & 31.97 & 45.36 & -121.90 & 524.75 & -322.39 & 0.70 \\
\hline SLy4 [207] & 0.160 & -15.97 & 229.91 & 363.11 & 32.00 & 45.94 & -119.73 & 521.53 & -322.83 & 0.69 \\
\hline SLy5 [207] & 0.161 & -15.99 & 229.92 & 364.16 & 32.01 & 48.15 & -112.76 & 500.67 & -325.38 & 0.70 \\
\hline SLy6 [207] & 0.159 & -15.92 & 229.86 & 360.24 & 31.96 & 47.45 & -112.71 & 510.63 & -323.03 & 0.69 \\
\hline SLy7 [207] & 0.158 & -15.90 & 229.75 & 359.22 & 31.99 & 46.94 & -114.34 & 517.14 & -322.60 & 0.69 \\
\hline SLy8 [206] & 0.160 & -15.97 & 229.89 & 363.27 & 32.00 & 47.18 & -115.59 & 509.88 & -324.09 & 0.70 \\
\hline SLy9 [206] & 0.151 & -15.80 & 229.84 & 350.42 & 31.98 & 54.86 & -81.42 & 462.35 & -326.92 & 0.67 \\
\hline SLy10 [207] & 0.156 & -15.90 & 229.68 & 358.32 & 31.90 & 38.51 & -142.18 & 591.23 & -313.17 & 0.68 \\
\hline SQMC1 [156] & 0.137 & -14.00 & 328.76 & -143.78 & 29.68 & -6.70 & -504.25 & 218.08 & -461.10 & 0.93 \\
\hline SQMC2 [156] & 0.140 & -14.29 & 330.10 & -121.75 & 28.70 & 8.67 & -408.41 & 145.55 & -463.63 & 0.83 \\
\hline SQMC3 [156] & 0.161 & -15.98 & 366.97 & -130.22 & 45.78 & 91.80 & -210.95 & 163.48 & -794.33 & 0.82 \\
\hline SQMC600 [157] & 0.174 & -15.74 & 217.00 & 388.62 & 34.38 & 46.38 & -215.16 & 396.85 & -410.40 & 0.81 \\
\hline SQMC650 [157] & 0.172 & -15.57 & 218.11 & 376.75 & 33.65 & 52.92 & -173.15 & 349.74 & -399.28 & 0.78 \\
\hline SQMC700 [157] & 0.171 & -15.49 & 222.20 & 369.94 & 33.47 & 59.06 & -140.84 & 313.84 & -396.85 & 0.76 \\
\hline SQMC750 [157] & 0.171 & -15.60 & 222.86 & 365.83 & 33.75 & 64.67 & -117.51 & 288.41 & -399.38 & 0.74 \\
\hline SSk [51] & 0.161 & -16.16 & 229.31 & 375.38 & 33.50 & 52.78 & -119.15 & 482.24 & -349.42 & 0.72 \\
\hline SV [183] & 0.155 & -16.05 & 305.70 & 175.78 & 32.82 & 96.09 & 24.17 & 48.00 & -497.11 & 0.38 \\
\hline SV-bas [115] & 0.160 & -15.91 & 233.45 & 379.28 & 30.00 & 32.37 & -221.75 & 410.93 & -363.36 & 0.90 \\
\hline SV-min [115] & 0.161 & -15.91 & 221.76 & 403.08 & 30.66 & 44.81 & -156.57 & 389.56 & -343.99 & 0.95 \\
\hline SVI [183] & 0.143 & -15.76 & 363.64 & -153.50 & 26.88 & -7.34 & -471.30 & 146.04 & -424.18 & 0.95 \\
\hline SVII [184] & 0.143 & -15.79 & 366.44 & -164.51 & 26.96 & -10.16 & -488.90 & 149.74 & -423.36 & 1.00 \\
\hline
\end{tabular}


TABLE II. (Continued.)

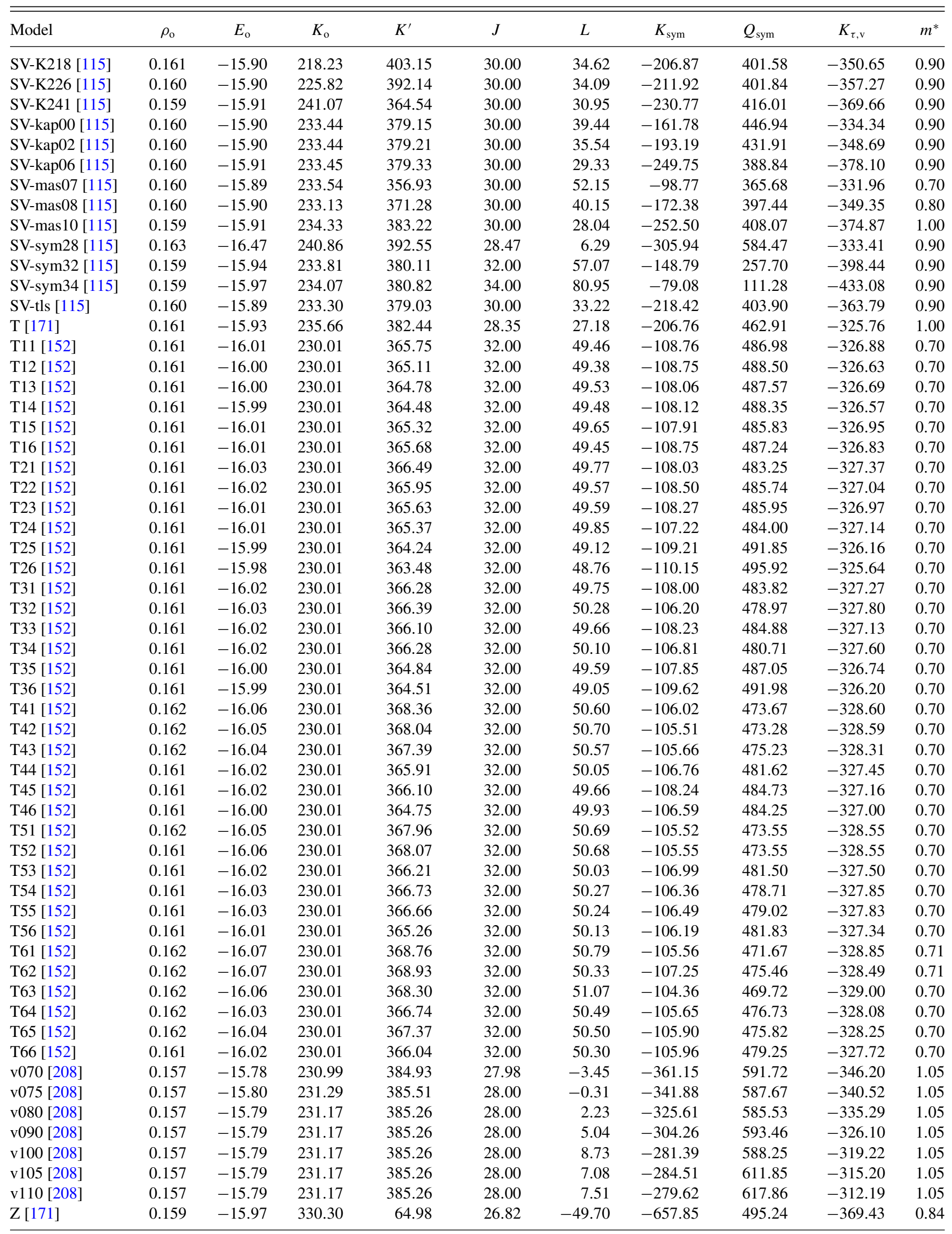


TABLE II. (Continued.)

\begin{tabular}{|c|c|c|c|c|c|c|c|c|c|c|}
\hline ZR1a [178] & 0.173 & -16.99 & 398.74 & -186.01 & 9.84 & -57.61 & -471.08 & 103.34 & -98.56 & 1.00 \\
\hline ZR1b [178] & 0.173 & -16.99 & 398.74 & -186.01 & 18.50 & -31.62 & -471.08 & 103.34 & -266.61 & 1.00 \\
\hline ZR2a [178] & 0.173 & -16.99 & 324.78 & 184.94 & 1.62 & -82.36 & -397.29 & 474.98 & 49.99 & 1.00 \\
\hline ZR2b [178] & 0.173 & -16.99 & 324.78 & 184.94 & 11.95 & -51.39 & -397.29 & 474.98 & -118.22 & 1.00 \\
\hline ZR3b [178] & 0.175 & -16.99 & 198.79 & 475.65 & -100.46 & -388.91 & -271.89 & 768.04 & 1131.04 & 1.00 \\
\hline ZR3c [178] & 0.175 & -16.99 & 198.79 & 475.65 & -42.71 & -215.66 & -271.89 & 768.04 & 506.06 & 1.00 \\
\hline Zs [171] & 0.163 & -15.88 & 233.33 & 368.95 & 26.69 & -29.38 & -401.43 & 883.05 & -271.61 & 0.78 \\
\hline $\mathrm{Zs}^{*}[171]$ & 0.162 & -15.96 & 234.87 & 369.16 & 28.80 & -4.53 & -332.64 & 725.10 & -312.58 & 0.77 \\
\hline
\end{tabular}

Further, considering the need to choose ranges for some constraints, we looked for sets that fell outside the chosen range for only one of the eleven constraints and by less then $5 \%$ of the closest limit. This procedure yielded ten more sets GSkI, GSkII, KDE0v1, MSL0, Ska35s20, SKRA, SkT1, SkT2, SkT3, and Skxs20, making a total of sixteen consistent Skyrme parameterizations (hereafter CSkP) which satisfy all constraints on properties of nuclear matter. (We note that sets SkT1a, SkT2a, and SkT3a (see Table II) have the same parameters relating to nuclear matter as SkT1, SkT2, and SkT3 [113] and differ only by a choice of the spin-orbit functional, the Coulomb exchange term, and the fitted pairing strength. SkT1a, SkT2a, and SkT3a are therefore not included separately in the analysis).

The values of all relevant parameters of the CSkP are given in Table VI. Table VII lists all the numerical properties of nuclear matter at saturation as calculated by the CSkP. The compliance of the CSkP with graphical constraints SM3, SM4, PNM1, and PNM2 is illustrated in Figs. 1-5.

The range of calculated values from all the CSkP is compared with the range of each constraint in Table I, where,

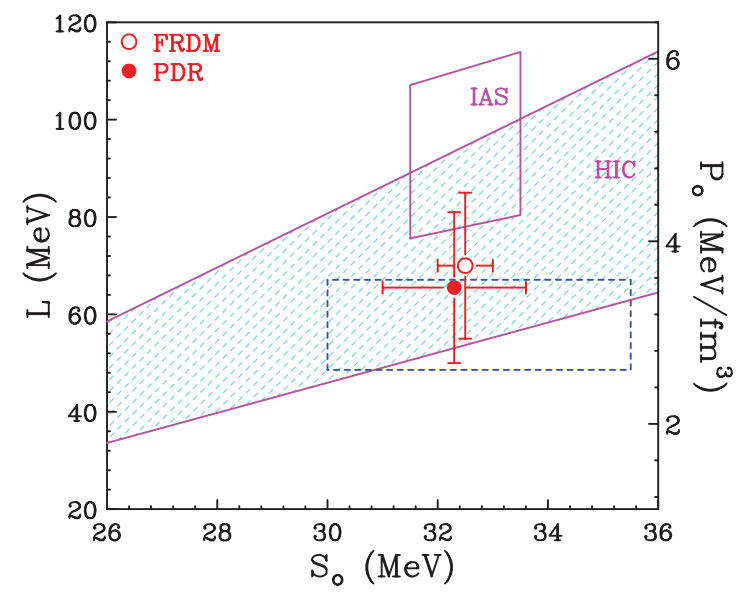

FIG. 6. (Color online) Constraints on symmetry energy $\mathcal{S}_{\mathrm{o}}$, its first derivative $L$, and $P_{\mathrm{o}}=P_{\mathrm{PNM}}\left(\rho_{\mathrm{o}}\right)$, all at saturation density, as derived from HIC [90], PDR [91,93], IAS [95], and FRDM [89]. Predictions of the consistent Skyrme parametrizations all lie within the blue dashed rectangle. For full explanation see text and [97]. it is interesting to note, they often fall within a band much narrower than the imposed constraint. Figures 1-5 show generally the same behavior for constraints defined by a function. Two exceptions concern constraint PNM1 (Fig. 3), where a narrow band is predicted by all models except KDE0v1 (high) and MSL0 (low), and MIX5 (Table VII), for which all results are in the upper half of the range, although not closely clustered.

As shown in Fig. 4(b), where the new band from Fig. 4(a) was added to the bands and curves already presented in Fig. 3, two models, KDE0v1 and MSL0, do not satisfy the more stringent theoretical constraint. However, as we consider PNM1 a valid constraint in the context of this paper, we keep the KDE0v1 and MSL0 parametrizations in the CSkP list. This issue will be revisited in future.

The relationship among $L, \mathcal{S}_{\mathrm{o}}=J$, and pressure in pure neutron matter, $P_{\mathrm{o}}$, has been examined by Tsang et al. [90]. Based on results of mass measurements, HIC, PDR, and IAS, they produced a composite constraint on these variables, which is an extended variation of our constraint MIX5. Figure 6 (taken from [97] and references therein) is the latest version of their Fig. 3 in [90]. Predictions of the CSkP of this relationship all fall within the blue dashed rectangle, overlapping with all the constraints but showing no compatibility with the IAS analysis.

\section{MICROSCOPIC AND OBSERVATIONAL CONSTRAINTS}

In addition to the eleven macroscopic constraints considered in previous sections, we introduce some additional, more microscopic constraints and constraints based on observation of neutron stars and apply them only to already-chosen CSkP. We find that these constraints significantly reduce further the number of the CSkP, eliminating GSkI, GSkII, MSL0, Ska25s20, Ska35s20, the SkT group, Skxs20, SQMC650, and SV-sym 32 from the CSkP list, as discussed in detail below.

\section{A. The effective mass}

In nonrelativistic models of the motion of a nucleon with mass $M$ in homogeneous nuclear matter, the nuclear potential 


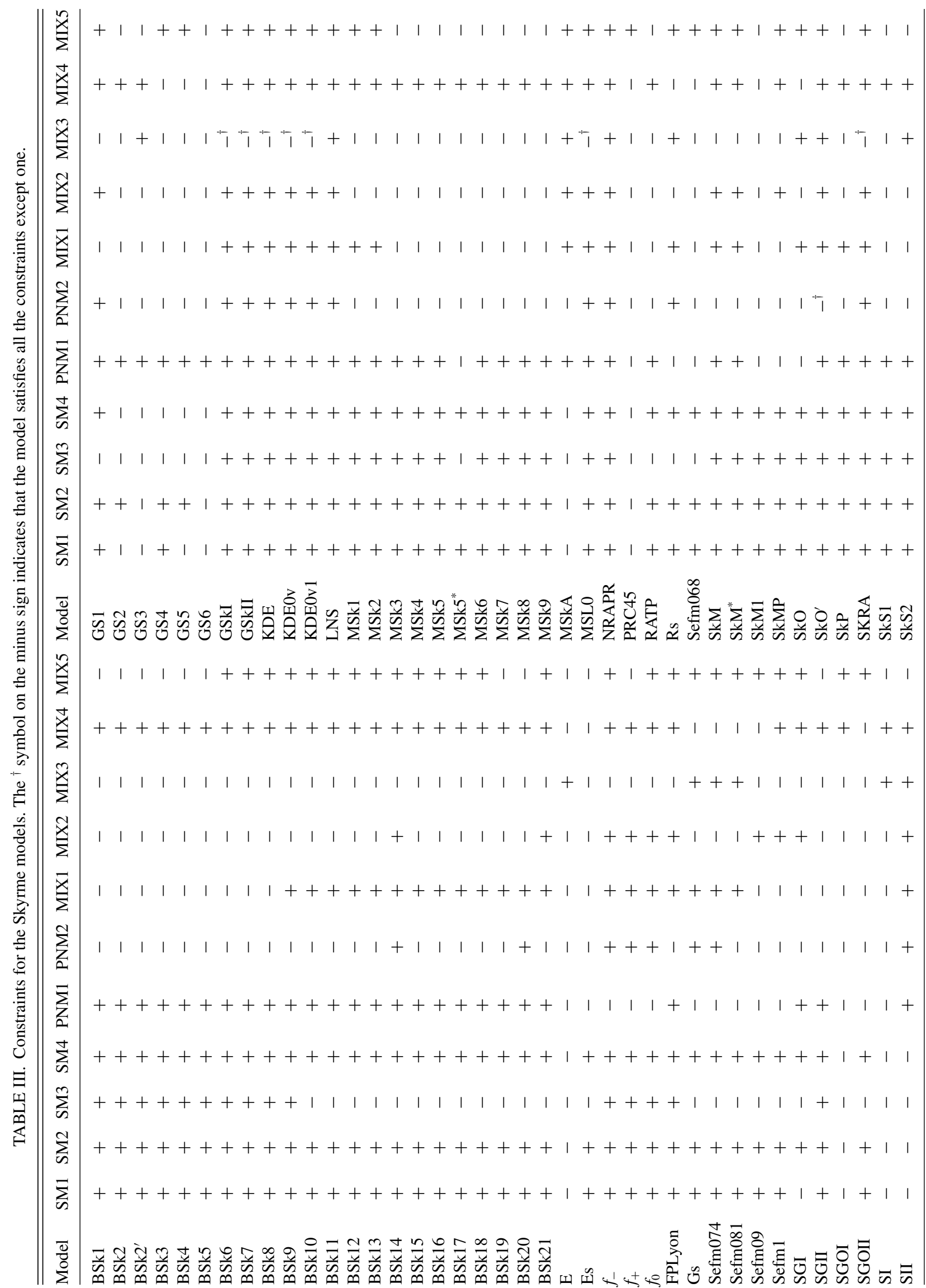




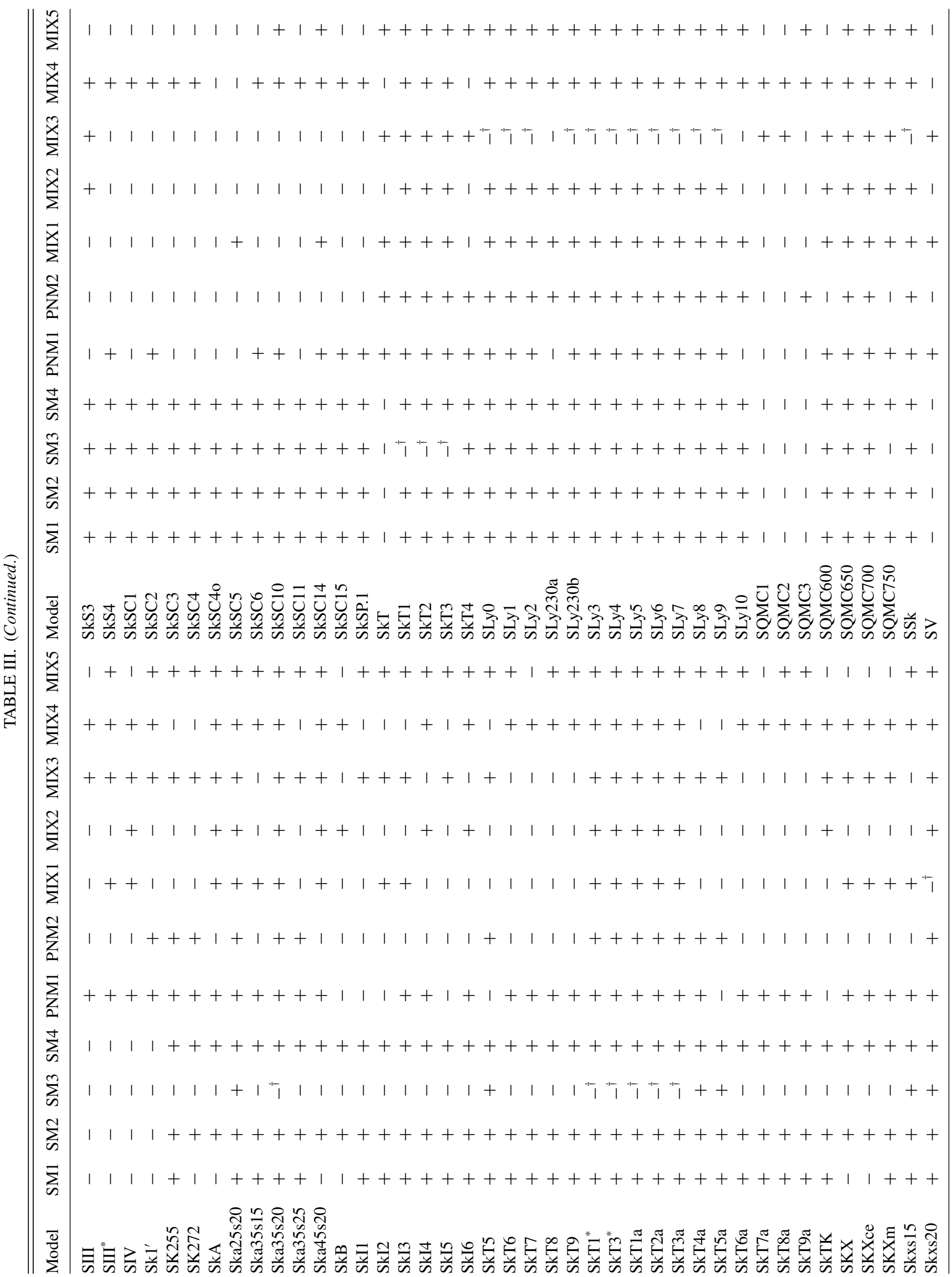




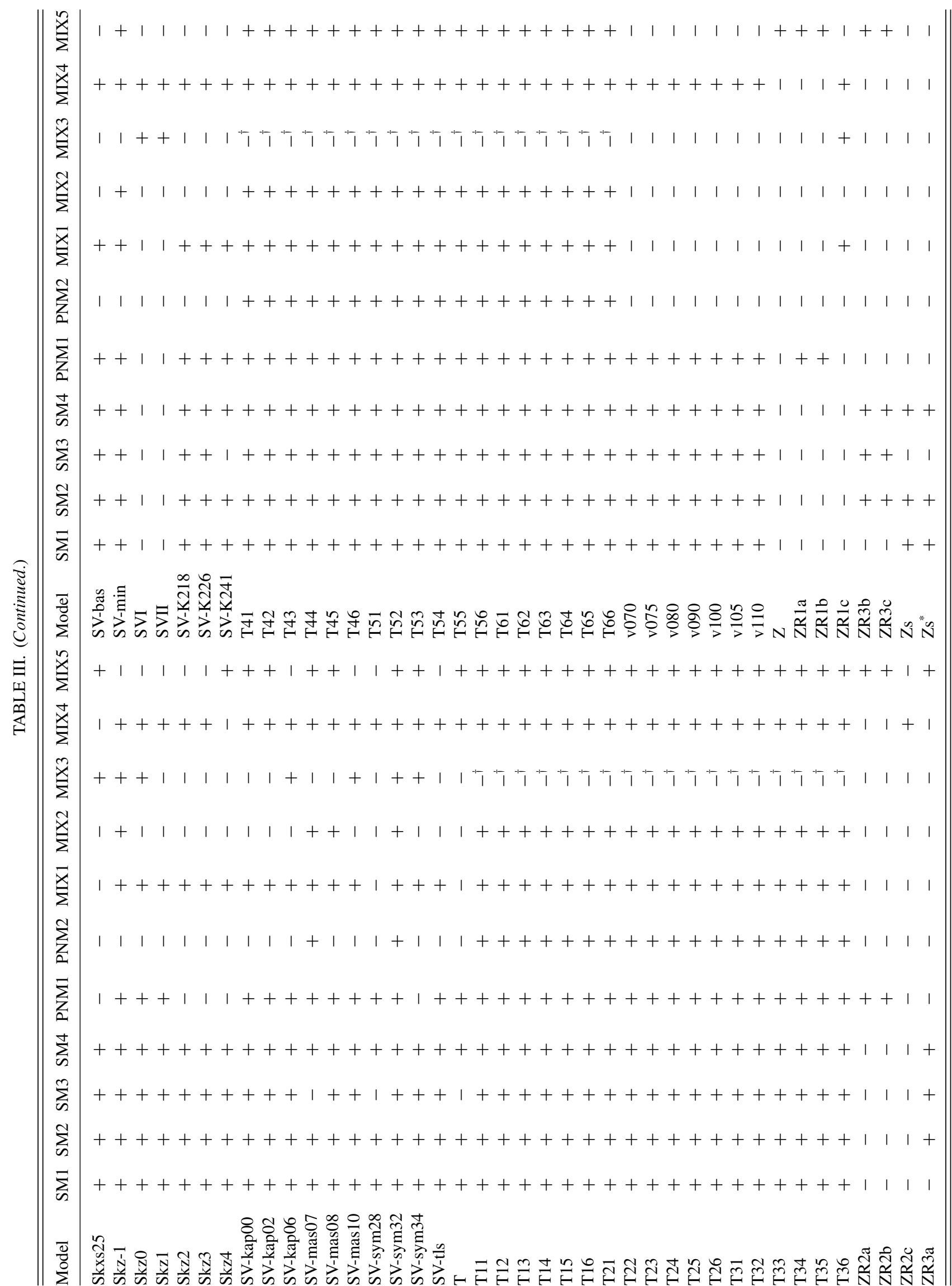


TABLE IV. List of the Skyrme parametrizations and their compliance with macroscopic constraints used in this work. For SM1, SM2, and MIX1-5 we give the standard deviation; the constraint is satisfied if the standard deviation is less than or equal to one. For SM3, SM4, PNM1, and PNM2 we give the fraction of full density range in which the constraint is not satisfied (in percent). Letter L (H) indicates that the Skyrme prediction starts to fail in the beginning (end) of the density range. No indication of letters means that the model fails in the middle of the density range. For models that fail in $100 \%$, the letter U (D) indicates that its curve is above (below) the band defined by the constraint. The ${ }^{\dagger}$ symbol marks a constraint which is the only one not satisfied (out of 11) by a particular Skyrme parametrization. For more details see text.

\begin{tabular}{|c|c|c|c|c|c|c|c|c|c|c|c|}
\hline Model & SM1 & SM2 & SM3 $(\%)$ & SM4 (\%) & PNM1 (\%) & PNM2 (\%) & MIX1 & MIX2 & MIX3 & MIX4 & MIX5 \\
\hline BSk1 & 0.04 & -0.63 & 0 & 0 & 0 & $100 \mathrm{D}$ & -1.88 & -2.82 & 1.30 & 0.69 & 1.70 \\
\hline BSk2 & 0.12 & -0.64 & 0 & 0 & 0 & $100 \mathrm{D}$ & -1.80 & -2.78 & 1.21 & 0.69 & 3.80 \\
\hline BSk3 & 0.16 & -0.64 & 0 & 0 & 0 & $100 \mathrm{D}$ & -1.83 & -2.85 & 1.18 & 0.69 & 4.40 \\
\hline BSk4 & 0.23 & -0.67 & $2.0 \mathrm{~L}$ & 0 & 0 & 100D & -1.80 & -2.53 & 1.26 & 0.54 & 2.00 \\
\hline BSk5 & 0.24 & -0.66 & $2.7 \mathrm{~L}$ & 0 & 0 & $100 \mathrm{D}$ & -1.52 & -2.03 & 1.19 & 0.31 & 1.20 \\
\hline BSk7 & -0.02 & -0.66 & 0 & 0 & 0 & 100D & -1.80 & -2.22 & 1.43 & 0.54 & 0.80 \\
\hline BSk8 & 0.01 & -0.66 & 0 & 0 & 2.4 & $100 \mathrm{D}$ & -1.80 & -2.40 & 1.44 & 0.62 & 0.90 \\
\hline BSk9 & 0.04 & -0.65 & 0 & 0 & 0 & $67.8 \mathrm{~L}$ & -1.00 & -1.09 & 1.26 & 0.00 & 0.30 \\
\hline BSk10 & 0.29 & -0.66 & $9.6 \mathrm{~L}$ & 0 & 0 & $100 \mathrm{D}$ & -1.00 & -1.15 & 1.06 & -0.08 & 0.80 \\
\hline BSk11 & 0.27 & -0.66 & $7.0 \mathrm{~L}$ & 0 & 0 & $100 \mathrm{D}$ & -1.00 & -1.09 & 1.06 & -0.08 & 0.70 \\
\hline BSk12 & 0.27 & -0.66 & $6.9 \mathrm{~L}$ & 0 & 0 & $100 \mathrm{D}$ & -1.00 & -1.11 & 1.06 & -0.08 & 0.80 \\
\hline BSk17 & 0.39 & -0.67 & $14.0 \mathrm{~L}$ & 0 & 0 & $100 \mathrm{D}$ & -1.00 & -1.21 & 1.14 & 0.00 & 0.70 \\
\hline BSk18 & 0.39 & -0.67 & $14.1 \mathrm{~L}$ & 0 & 0 & $100 \mathrm{D}$ & -1.00 & -1.21 & 1.15 & 0.00 & 0.70 \\
\hline BSk19 & 0.24 & -0.80 & $23.6 \mathrm{~L}$ & 0 & 0 & $100 \mathrm{D}$ & -1.00 & -1.45 & 1.15 & 0.15 & 1.90 \\
\hline BSk20 & 0.38 & -0.84 & $30.6 \mathrm{~L}$ & 0 & 0 & 0.0 & -1.00 & -1.15 & 1.28 & 0.08 & 1.30 \\
\hline BSk21 & 0.53 & -0.85 & $33.4 \mathrm{~L}$ & 0 & 0 & $17.1 \mathrm{~L}$ & -1.00 & -0.64 & 1.55 & 0.00 & 0.70 \\
\hline $\mathrm{E}$ & 3.45 & -1.27 & $100 \mathrm{U}$ & $100 \mathrm{U}$ & $100 \mathrm{U}$ & $100 \mathrm{D}$ & -1.94 & -4.96 & 0.91 & 1.46 & -1.20 \\
\hline Es & 0.62 & -0.70 & $29.2 \mathrm{~L}$ & 0 & $100 \mathrm{U}$ & $100 \mathrm{D}$ & -2.42 & -5.27 & 1.43 & 2.00 & -1.20 \\
\hline$f_{-}$ & 0.00 & -0.59 & 0 & 0 & $15.4 \mathrm{~L} / 11.2 \mathrm{H}$ & 0 & -0.20 & -0.79 & 1.42 & 0.00 & 0.10 \\
\hline$f_{+}$ & 0.00 & -0.59 & 0 & 0 & $19.0 \mathrm{~L} / 25.8 \mathrm{H}$ & 0 & -0.20 & -0.91 & 1.40 & 0.08 & 1.10 \\
\hline$f_{0}$ & 0.00 & -0.59 & 0 & 0 & $16.8 \mathrm{~L} / 18.8 \mathrm{H}$ & 0 & -0.20 & -0.87 & 1.41 & 0.08 & 0.70 \\
\hline FPLyon & -0.43 & -0.60 & 0 & 0 & 0 & $8.2 \mathrm{~L}$ & -0.63 & -0.85 & 1.30 & -0.08 & 0.20 \\
\hline GSkI & 0.01 & -0.59 & 0 & 0 & 0 & $0.7 \mathrm{~L}$ & -0.19 & 0.30 & $1.04^{\dagger}$ & -0.62 & 0.20 \\
\hline GSkII & 0.11 & -0.60 & 0 & 0 & 0 & 4.0 & -0.80 & -0.52 & $1.03^{\dagger}$ & -0.38 & 0.90 \\
\hline KDE & -0.20 & -0.64 & 0 & 0 & 0 & 0 & -0.21 & -0.92 & $1.27^{\dagger}$ & 0.00 & 0.30 \\
\hline KDE0v & -0.04 & -0.65 & 0 & 0 & 0 & 0 & 0.19 & -0.71 & $1.15^{\dagger}$ & -0.08 & 0.40 \\
\hline KDE0v1 & -0.08 & -0.63 & 0 & 0 & 0 & 0 & 0.83 & -0.18 & $1.05^{\dagger}$ & -0.23 & 0.30 \\
\hline LNS & -0.64 & -0.63 & 0 & 0 & 0 & 0 & 0.37 & 0.19 & 0.94 & -0.54 & 0.60 \\
\hline MSk1 & 0.12 & -0.64 & 0.0 & 0 & 0 & $100 \mathrm{D}$ & -1.00 & -1.34 & 1.12 & 0.00 & 0.40 \\
\hline MSk2 & 0.06 & -0.63 & 0.0 & 0 & 0 & $100 \mathrm{D}$ & -1.00 & -1.37 & 1.12 & 0.08 & 0.40 \\
\hline MSk3 & 0.11 & -0.64 & 0.0 & 0 & 0 & $100 \mathrm{D}$ & -1.80 & -2.83 & 1.30 & 0.77 & 2.00 \\
\hline MSk4 & 0.04 & -0.63 & 0.0 & 0 & 0 & $100 \mathrm{D}$ & -1.80 & -2.82 & 1.29 & 0.69 & 1.70 \\
\hline MSk5 & 0.04 & -0.63 & 0.0 & 0 & 0 & $100 \mathrm{D}$ & -1.80 & -2.80 & 1.29 & 0.69 & 1.60 \\
\hline MSk5* & 0.46 & -0.71 & $14.9 \mathrm{~L}$ & 0 & $6.4 \mathrm{H}$ & $100 \mathrm{D}$ & -1.80 & -2.83 & 1.25 & 0.69 & 5.40 \\
\hline MSk6 & 0.04 & -0.63 & 0.0 & 0 & 0 & 100D & -1.80 & -2.69 & 1.29 & 0.69 & 1.30 \\
\hline MSk7 & 0.04 & -0.63 & 0.0 & 0 & 0 & $100 \mathrm{D}$ & -1.82 & -2.70 & 1.29 & 0.69 & 1.30 \\
\hline MSk8 & -0.02 & -0.62 & 0 & 0 & 0 & $100 \mathrm{D}$ & -1.83 & -2.76 & 1.29 & 0.69 & 1.30 \\
\hline MSk9 & 0.11 & -0.64 & 0 & 0 & 0 & $100 \mathrm{D}$ & -1.80 & -2.65 & 1.29 & 0.62 & 1.30 \\
\hline MSkA & 2.78 & -1.12 & $100 \mathrm{U}$ & $100 \mathrm{U}$ & 0 & $48.4 \mathrm{~L}$ & -0.86 & -0.05 & 0.58 & -0.62 & 0.70 \\
\hline
\end{tabular}


TABLE IV. (Continued.)

\begin{tabular}{|c|c|c|c|c|c|c|c|c|c|c|c|}
\hline MSL0 & 0.00 & -0.64 & 0 & 0 & 0 & 0 & -1.00 & 0.11 & $1.06^{\dagger}$ & -0.69 & 0.60 \\
\hline NRAPR & -0.14 & -0.67 & 0 & 0 & 0 & 0 & 0.11 & 0.09 & 0.93 & -0.54 & 0.80 \\
\hline RATP & 0.32 & -0.70 & $16.3 \mathrm{~L}$ & 0 & 0 & $100 \mathrm{D}$ & -1.30 & -1.42 & 1.17 & 0.08 & 1.70 \\
\hline Rs & 0.25 & -0.70 & $8.4 \mathrm{~L}$ & 0 & $86.4 \mathrm{H}$ & 0 & -0.67 & 1.58 & 0.85 & -1.31 & 0.50 \\
\hline Sefm081 & 0.23 & -0.69 & $12.6 \mathrm{~L}$ & 0.0 & 80.2 & $15.4 \mathrm{~L}$ & -0.70 & 1.19 & 0.87 & -1.15 & 0.50 \\
\hline Sefm09 & 0.34 & -0.70 & $17.3 \mathrm{~L}$ & 0.0 & $80.2 \mathrm{H}$ & $59.4 \mathrm{~L}$ & -1.89 & 0.66 & 1.07 & -1.08 & 0.50 \\
\hline Sefm1 & 0.34 & -0.71 & $17.8 \mathrm{~L}$ & 0.0 & $80.8 \mathrm{H}$ & $38.8 \mathrm{~L}$ & -3.08 & 0.09 & 1.28 & -1.00 & 0.50 \\
\hline SGI & 1.06 & -0.80 & $37.6 \mathrm{~L}$ & 0 & 0 & $52.6 \mathrm{~L}$ & -1.67 & 0.33 & 1.05 & -0.85 & 0.70 \\
\hline SGII & -0.51 & -0.64 & 0 & 0 & 0 & $100 \mathrm{D}$ & -2.27 & -1.13 & 1.35 & -0.15 & 1.10 \\
\hline SGOI & 4.39 & -1.47 & $100 \mathrm{U}$ & $100 U$ & $10.0 \mathrm{~L} / 46.4 \mathrm{H}$ & $100 \mathrm{U}$ & 5.08 & 2.32 & -1.02 & -0.92 & 0.70 \\
\hline SIII* & 4.37 & -1.62 & $100 \mathrm{U}$ & $100 \mathrm{U}$ & 0.0 & $100 \mathrm{D}$ & -0.21 & -1.63 & 0.14 & -0.08 & 1.00 \\
\hline SIV & 3.15 & -1.26 & $100 \mathrm{U}$ & $100 U$ & 0 & $54.7 \mathrm{~L}$ & -0.51 & 0.31 & 0.32 & -0.77 & 1.30 \\
\hline $\mathrm{Sk} 1^{\prime}$ & 4.68 & -1.70 & $100 \mathrm{U}$ & $100 U$ & 0 & 0 & -1.26 & -1.26 & 0.41 & -0.23 & 0.90 \\
\hline SK255 & 0.83 & -0.70 & $28.7 \mathrm{~L}$ & 0 & 0 & 0.0 & 1.96 & 2.06 & 0.35 & -1.15 & 0.50 \\
\hline SK272 & 1.38 & -0.79 & $45.1 \mathrm{~L}$ & 0 & 0 & 0.0 & 1.96 & 1.87 & 0.26 & -1.08 & 0.50 \\
\hline SkA & 1.11 & -0.80 & $39.6 \mathrm{~L}$ & 0 & 0 & $42.7 \mathrm{~L}$ & 0.16 & 0.92 & 0.64 & -0.92 & 0.80 \\
\hline Ska25s20 & -0.31 & -0.57 & 0 & 0 & 0 & 0 & 0.51 & 0.32 & 0.95 & -0.54 & 0.20 \\
\hline Ska35s15 & 0.30 & -0.64 & $5.1 \mathrm{~L}$ & 0 & 0 & $100 \mathrm{D}$ & -0.78 & -1.52 & 1.07 & 0.15 & 0.40 \\
\hline Ska35s20 & 0.34 & -0.64 & $7.6 \mathrm{~L}^{\dagger}$ & 0 & 0 & 0 & 0.43 & 0.38 & 0.82 & -0.62 & 0.20 \\
\hline Ska35s25 & 0.38 & -0.64 & $8.5 \mathrm{~L}$ & 0 & 0 & 0 & 1.79 & 2.27 & 0.54 & -1.23 & 0.10 \\
\hline Ska45s20 & 1.01 & -0.74 & $33.6 \mathrm{~L}$ & 0 & 0 & $12.5 \mathrm{~L}$ & 0.36 & 0.46 & 0.68 & -0.69 & 0.20 \\
\hline SkM & -0.45 & -0.63 & 0 & 0 & 0 & 5.6 & -0.70 & -0.48 & 1.08 & -0.38 & 0.90 \\
\hline $\mathrm{SkM}^{*}$ & -0.45 & -0.63 & 0 & 0 & 0 & $100 \mathrm{D}$ & -0.99 & -0.68 & 1.12 & -0.31 & 1.00 \\
\hline SkM1 & -0.45 & -0.63 & 0.0 & 0 & $100 \mathrm{U}$ & $100 \mathrm{D}$ & -2.93 & -5.19 & 1.68 & 2.15 & -1.30 \\
\hline SkMP & 0.03 & -0.72 & 0 & 0 & 40.0 & $55.2 \mathrm{~L}$ & -1.04 & 0.68 & 1.02 & -0.92 & 0.70 \\
\hline $\mathrm{SkO}$ & -0.22 & -0.61 & 0 & 0 & 64.0 & $57.1 \mathrm{~L}$ & -0.21 & 1.17 & 0.96 & -1.08 & 0.30 \\
\hline $\mathrm{SkO}^{\prime}$ & -0.25 & -0.62 & 0 & 0 & 0 & $23.6 \mathrm{~L}^{\dagger}$ & -0.22 & 0.61 & 1.00 & -0.77 & 0.30 \\
\hline $\mathrm{SkP}$ & -0.97 & -0.53 & 0 & 0 & 0 & $100 \mathrm{D}$ & -1.00 & -2.13 & 1.15 & 0.38 & 2.30 \\
\hline SKRA & -0.43 & -0.64 & 0 & 0 & 0 & 0 & -0.47 & -0.28 & $1.04^{\dagger}$ & -0.38 & 0.90 \\
\hline SkS1 & -0.05 & -0.63 & 0 & 0 & 0 & $100 \mathrm{D}$ & -1.50 & -1.53 & 1.11 & 0.00 & 1.80 \\
\hline $\mathrm{SkS} 2$ & -0.03 & -0.63 & 0 & 0 & 0 & $100 \mathrm{D}$ & -1.31 & -1.12 & 0.95 & -0.23 & 2.00 \\
\hline $\mathrm{SkS} 3$ & -0.04 & -0.63 & 0 & 0 & 54.2 & $56.4 \mathrm{H}$ & -1.46 & -0.35 & 0.95 & -0.62 & 1.40 \\
\hline $\mathrm{SkS} 4$ & -0.06 & -0.63 & 0 & 0 & 0.0 & $100 \mathrm{D}$ & -1.66 & -1.93 & 1.17 & 0.23 & 2.20 \\
\hline SkSC1 & 0.15 & -0.64 & $2.5 \mathrm{~L}$ & 0 & $20.0 \mathrm{~L} / 67.8 \mathrm{H}$ & $100 \mathrm{D}$ & -1.76 & -3.21 & 1.31 & 0.92 & 5.00 \\
\hline SkSC2 & 0.17 & -0.64 & $3.4 \mathrm{~L}$ & 0 & 0.0 & $100 \mathrm{D}$ & -3.10 & -2.61 & 1.49 & 0.62 & 1.30 \\
\hline SkSC3 & 0.15 & -0.64 & $2.3 \mathrm{~L}$ & 0 & $10.8 \mathrm{H}$ & $100 \mathrm{D}$ & -2.20 & -3.18 & 1.37 & 0.92 & 17.40 \\
\hline SkSC4 & 0.16 & -0.64 & $2.7 \mathrm{~L}$ & 0 & $100 \mathrm{U}$ & $100 \mathrm{D}$ & -1.48 & -3.34 & 1.27 & 1.00 & -6.70 \\
\hline $\mathrm{SkSC} 4 \mathrm{o}$ & 0.16 & -0.64 & $3.0 \mathrm{~L}$ & 0 & $100 \mathrm{U}$ & $100 \mathrm{D}$ & -2.20 & -3.76 & 1.39 & 1.23 & -1.50 \\
\hline SkSC5 & 0.15 & -0.64 & $2.3 \mathrm{~L}$ & 0 & $100 \mathrm{U}$ & $100 \mathrm{D}$ & -0.60 & -3.61 & 1.14 & 1.08 & -2.00 \\
\hline SkSC6 & 0.18 & -0.64 & $4.1 \mathrm{~L}$ & 0 & 0 & $100 \mathrm{D}$ & -3.17 & -2.61 & 1.50 & 0.62 & 1.30 \\
\hline SkSC10 & 0.20 & -0.63 & $4.9 \mathrm{~L}$ & 0 & 0 & $100 \mathrm{D}$ & -3.87 & -2.16 & 1.60 & 0.31 & 0.70 \\
\hline SkSC11 & 0.16 & -0.64 & $2.7 \mathrm{~L}$ & 0 & $100 \mathrm{U}$ & $100 \mathrm{D}$ & -1.48 & -3.34 & 1.27 & 1.00 & -6.70 \\
\hline SkSC14 & 0.18 & -0.64 & $4.1 \mathrm{~L}$ & 0 & 0 & $100 \mathrm{D}$ & -1.00 & -1.38 & 1.12 & 0.08 & 0.40 \\
\hline
\end{tabular}


TABLE IV. (Continued.)

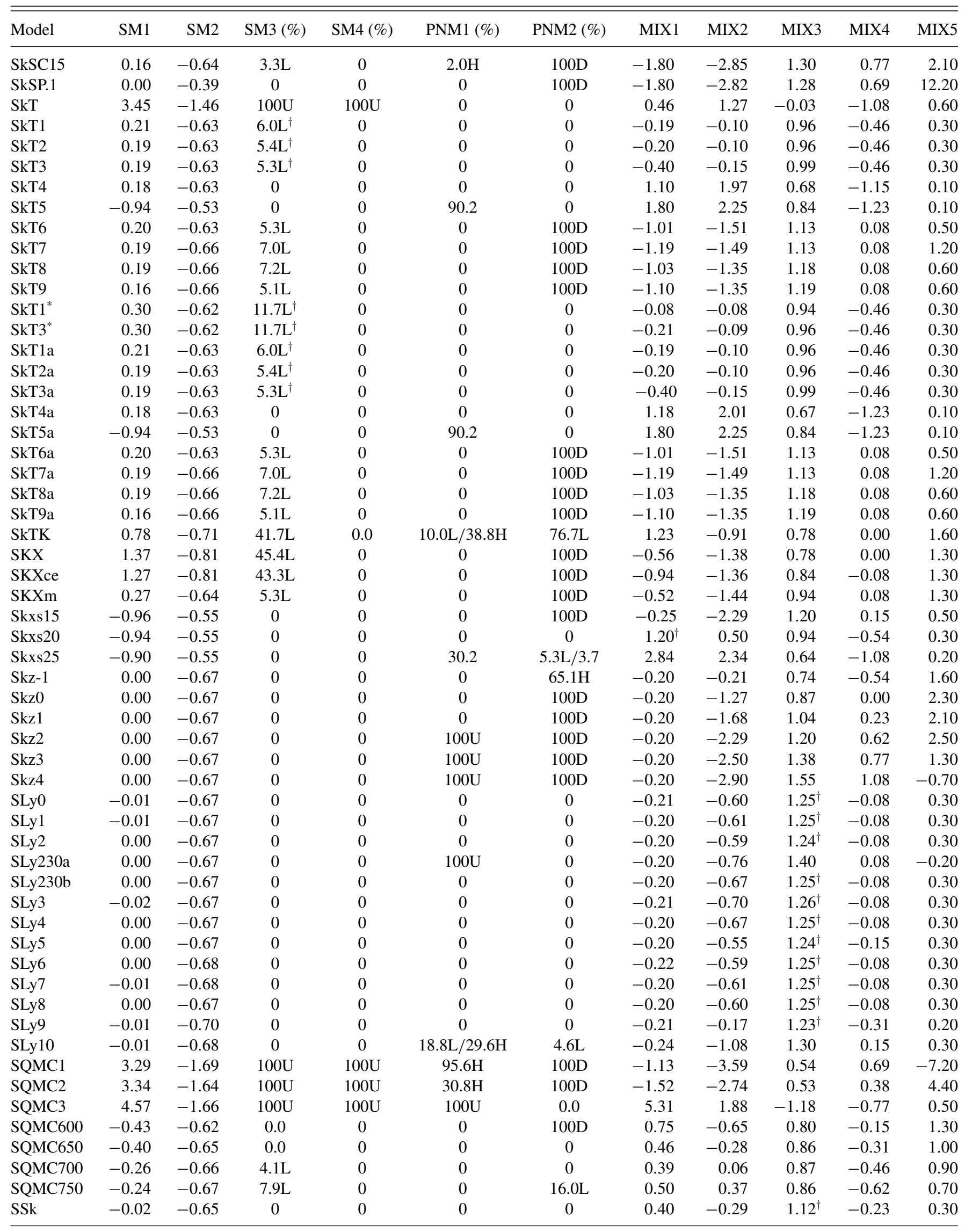


TABLE IV. (Continued.)

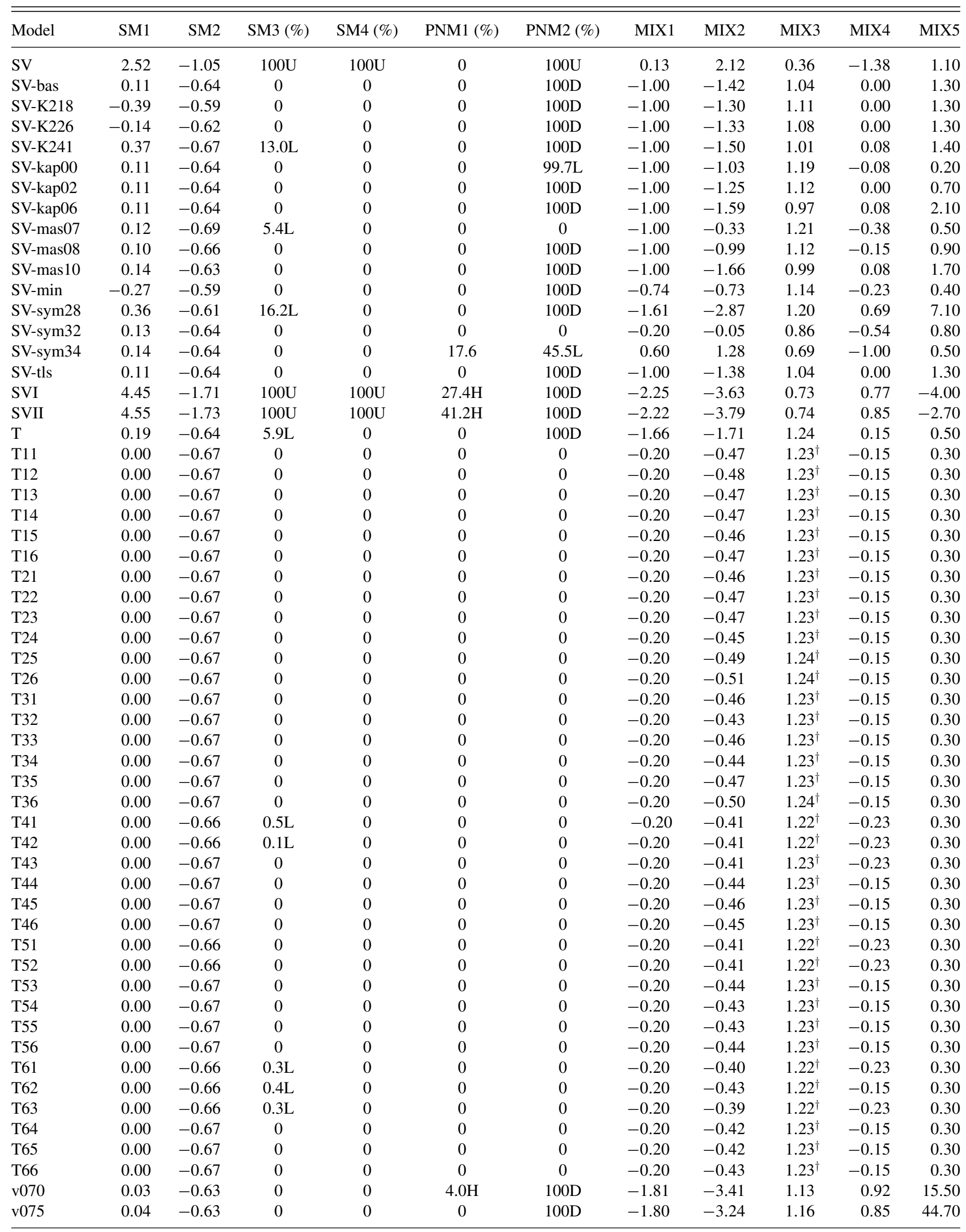


TABLE IV. (Continued.)

\begin{tabular}{|c|c|c|c|c|c|c|c|c|c|c|c|}
\hline v080 & 0.04 & -0.63 & 0 & 0 & 0 & 100D & -1.80 & -3.10 & 1.19 & 0.77 & 17.10 \\
\hline v090 & 0.04 & -0.63 & 0 & 0 & 0 & $100 \mathrm{D}$ & -1.80 & -2.94 & 1.24 & 0.77 & 5.20 \\
\hline v105 & 0.04 & -0.63 & 0 & 0 & 0 & 100D & -1.80 & -2.83 & 1.29 & 0.69 & 1.70 \\
\hline v110 & 0.04 & -0.63 & 0 & 0 & 0 & 100D & -1.80 & -2.81 & 1.31 & 0.69 & 1.10 \\
\hline Z & 3.34 & -1.27 & $100 \mathrm{U}$ & $100 \mathrm{U}$ & $100 \mathrm{U}$ & 100D & -2.27 & -5.98 & 1.01 & 1.92 & -0.70 \\
\hline ZR1b & 5.62 & -1.77 & $100 \mathrm{U}$ & $100 \mathrm{U}$ & $2.4 \mathrm{~L}$ & 100D & -5.60 & -4.98 & 1.54 & 1.69 & -0.50 \\
\hline ZR1c & 5.62 & -1.77 & $100 \mathrm{U}$ & $100 \mathrm{U}$ & $100 U$ & 100D & -0.40 & -2.81 & 0.24 & 0.38 & 2.00 \\
\hline ZR2a & 3.16 & -1.03 & $100 \mathrm{U}$ & $100 \mathrm{U}$ & 0 & $100 \mathrm{D}$ & 12.35 & -7.80 & 3.18 & 39.08 & -0.20 \\
\hline ZR2b & 3.16 & -1.03 & $100 \mathrm{U}$ & $100 \mathrm{U}$ & 0 & $100 \mathrm{D}$ & -8.22 & -6.08 & 2.31 & 4.00 & -0.30 \\
\hline ZR2c & 3.16 & -1.03 & $100 \mathrm{U}$ & $100 \mathrm{U}$ & $100 \mathrm{U}$ & $100 \mathrm{D}$ & -2.03 & -3.50 & 1.01 & 0.85 & -3.00 \\
\hline ZR3a & -1.04 & -0.45 & 0 & 0 & 100D & 100D & 68.58 & 31.25 & 10.90 & -2.08 & 0.00 \\
\hline
\end{tabular}

$V(k)$ is momentum dependent. The concept of the effective mass $M^{*}$, originally developed by Brueckner [114], leads to an equivalent description of the motion in which the nuclear potential $V(k=0)$ is constant but the nucleon mass has been modified. It has been established that $M^{*}$ is lower than $M$ for all potentials for which the low- $k$ expansion $V(k)=V(0)+$ $b k^{2}+\cdots$ (where $b$ is a constant) is valid.

This simple formalism can be extended also to momentumand density-dependent potentials, such as the Skyrme potential. The nucleon isoscalar effective mass $M_{s}^{*}$ at saturation density can be defined [115] as

$$
\frac{\hbar^{2}}{2 M_{s}^{*}}=\frac{\hbar^{2}}{2 M}+\left.\frac{\partial E}{\partial \tau}\right|_{\rho_{\mathrm{o}}},
$$

where $E$ is the energy per particle, Eq. (1), which leads to [45]

$$
m_{s}^{*}=\frac{M_{s}^{*}}{M}=\left(1+\frac{M}{8 \hbar^{2}} \rho_{\mathrm{o}} \Theta_{\mathrm{s}}\right)^{-1},
$$

in terms of the Skyrme parameters.

The isovector effective mass $M_{v}^{*}$ is given as

$$
m_{v}^{*}=\frac{M_{v}^{*}}{M}=\frac{1}{1+\kappa}=\left(1+\frac{M}{4 \hbar^{2}} \rho_{\mathrm{o}} \Theta_{\mathrm{v}}\right)^{-1},
$$

where $\kappa$ is the enhancement factor of the Thomas-ReicheKuhn sum rule [116],

$$
\kappa=\left.\frac{2 M}{\hbar^{2}} \rho_{\mathrm{o}} \frac{\partial}{\partial\left(\tau_{n}-\tau_{p}\right)} \frac{\partial}{\partial\left(\rho_{n}-\rho_{p}\right)} E\right|_{\rho_{\mathrm{o}}}
$$

(and we note the typographical error in [115] Eq. (14) reported in [117]). In the above equations $\tau, \tau_{p}$, and $\tau_{n}$ are the total, proton, and neutron kinetic energy densities and $\Theta_{\mathrm{s}}$ and $\Theta_{\mathrm{v}}$ are defined in Eq. (61).

In ANM, consisting of unequal amounts of neutrons and protons, the nucleon effective mass can be written in terms of $\Theta_{\mathrm{S}}$ and $\Theta_{\mathrm{v}}$ as $[113,118,119]$

$$
m_{q}^{*}=\frac{M_{q}^{*}}{M}=\left(1+\frac{M}{8 \hbar^{2}} \rho \Theta_{\mathrm{s}}-\frac{M}{8 \hbar^{2}} q\left(2 \Theta_{\mathrm{v}}-\Theta_{\mathrm{s}}\right) \beta \rho\right)^{-1}
$$

where $\beta$ is the asymmetry parameter, $\left(\rho_{n}-\rho_{p}\right) / \rho$, and $q=1$ $(-1)$ for neutrons (protons).

For nonstandard parametrizations, Eq. (48) becomes

$$
\begin{aligned}
m_{q}^{*}= & {\left[1+\frac{M}{8 \hbar^{2}} \rho \Theta_{\mathrm{s}}^{\prime}-\frac{M}{8 \hbar^{2}} q\left(2 \Theta_{\mathrm{v}}-\Theta_{\mathrm{s}}-t_{4}\left(1+2 x_{4}\right) \rho^{\beta}\right.\right.} \\
& \left.\left.+t_{5}\left(1+2 x_{5}\right) \rho^{\alpha}\right) \beta \rho\right]^{-1} .
\end{aligned}
$$

For such a case, $m_{s}^{*}$ and $m_{v}^{*}$ are obtained by making $\Theta_{\mathrm{s}} \rightarrow \Theta_{\mathrm{s}}^{\prime}$ and $\Theta_{\mathrm{v}} \rightarrow \Theta_{\mathrm{v}}^{\prime}$ in Eqs. (45) and (46), where $\Theta_{\mathrm{s}}^{\prime}$ and $\Theta_{\mathrm{v}}^{\prime}$ are defined in Eq. (63).

Constraints on $m_{s}^{*}$ and $m_{v}^{*}$ at saturation density can be derived from experimental peak frequencies of giant resonances in finite nuclei ([115,120] and references therein). $m_{s}^{*}$ is solely related to the giant quadrupole resonance (GQR). Klüpfel et al. [115] deduced an optimum value of $m_{s}^{*}=0.9$ from the GQR in ${ }^{208} \mathrm{~Pb}$, which is close to the estimate of Bohias

TABLE V. Number of Skyrme models consistent with individual macroscopic constraints.

\begin{tabular}{lcccccccccc}
\hline \hline Constraints & SM1 & SM2 & SM3 & SM4 & PNM1 & PNM2 & MIX1 & MIX2 & MIX3 & MIX4 \\
No. CSkP & 202 & 214 & 145 & 211 & 186 & 94 & 139 & 102 & 65 & 198 \\
\hline \hline
\end{tabular}


TABLE VI. Parameters of the Skyrme interactions consistent with the macroscopic constraints. $t_{0}$ is in $\mathrm{MeV}_{\mathrm{fm}}^{3}, t_{1} \mathrm{and}_{2}$ are in $\mathrm{MeV}_{\mathrm{fm}}{ }^{5}$, and $t_{3 i}$ is in $\mathrm{MeV} \mathrm{fm}{ }^{3+3 \sigma_{i}} . x_{0}, x_{1}, x_{2}, x_{3 i}$, and $\sigma_{i}$ are dimensionless. For all parametrizations $t_{4}=x_{4}=0$ and $t_{5}=x_{5}=0$.

\begin{tabular}{|c|c|c|c|c|c|c|c|c|c|c|c|c|c|c|c|}
\hline GSkI & -1855.5 & 397.2 & 264.6 & 13858.0 & -2694.1 & -319.9 & 0.12 & -1.76 & -1.81 & 0.13 & -1.19 & -0.46 & 0.33 & 0.67 & 1.00 \\
\hline GSkII & -1856.0 & 393.1 & 266.1 & 13842.9 & -2689.7 & - & 0.09 & -0.72 & -1.84 & -0.10 & -0.35 & - & 0.33 & 0.67 & - \\
\hline LNS & -2485.0 & 266.7 & -337.1 & 14588.2 & - & - & 0.06 & 0.66 & -0.95 & -0.03 & - & - & 0.17 & - & - \\
\hline MSL0 & -2118.1 & 395.2 & -64.0 & 12875.7 & - & - & -0.07 & -0.33 & 1.36 & -0.23 & - & - & 0.24 & - & - \\
\hline NRAPR & -2719.7 & 417.6 & -66.7 & 15042.0 & - & - & 0.16 & -0.05 & 0.03 & 0.14 & - & - & 0.14 & - & - \\
\hline Ska35s20 & -1768.8 & 263.9 & -158.3 & 12904.8 & - & - & 0.13 & -0.80 & 0.00 & 0.01 & - & - & 0.35 & - & - \\
\hline SKRA & -2895.4 & 405.5 & -89.1 & 16660.0 & - & - & 0.08 & 0.00 & 0.20 & 0.00 & - & - & 0.14 & - & - \\
\hline SkT1 & -1794.0 & 298.0 & -298.0 & 12812.0 & - & - & 0.15 & -0.50 & -0.50 & 0.09 & - & - & 0.33 & - & - \\
\hline SkT2 & -1791.6 & 300.0 & -300.0 & 12792.0 & - & - & 0.15 & -0.50 & -0.50 & 0.09 & - & - & 0.33 & - & - \\
\hline SkT3 & -1791.8 & 298.5 & -99.5 & 12794.0 & - & - & 0.14 & -1.00 & 1.00 & 0.08 & - & - & 0.33 & - & - \\
\hline Skxs20 & -2885.2 & 302.7 & -323.4 & 18237.5 & - & - & 0.14 & -0.26 & -0.61 & 0.05 & - & - & 0.17 & - & - \\
\hline
\end{tabular}

et al. [121] of $m_{s}^{*}=0.8 . m_{v}^{*}$ is constrained from the giant dipole resonance (GDR), which is sensitive to two nuclear matter variables, the symmetry energy and the enhancement factor $\kappa$. Klüpfel et al., taking $\kappa=0.4$, obtained $m_{v}^{*}=0.7$, the same as the value extracted in [122]. However, these values, based on the GDR in ${ }^{208} \mathrm{~Pb}$, are not quite consistent with data of the GDR in ${ }^{16} \mathrm{O}$ [123]. The current conclusion is that experimental GDR data on light and heavy nuclei cannot be satisfied simultaneously with the present form of the static $\mathrm{HF}$ functional. Additional work is needed in both theory and experiment.

The effective mass scales the level density $g$ of singleparticle (s.p.) levels in the vicinity of the Fermi surface $g\left(\epsilon_{F}\right) \rightarrow \frac{M}{M^{*}} g\left(\epsilon_{F}\right)$ (see [124] and references therein). This simple scaling, which is valid in infinite matter, holds for the level density of deep s.p. states in finite nuclei, but it breaks down for s.p. states close to the Fermi level. The origin of the change in level density is usually seen in coupling between HF s.p. modes and surface-vibration (beyond HF) RPA modes (see, e.g., [125]). If the coupling is taken into account, the nuclear matter effective mass scaling can be recovered. Such calculation is however rather complicated and the simplest way to fit experimental s.p. level density is to take $M^{*}$ being state dependent and equal to $M$ at the Fermi surface [119]. Such an approach is also necessary in fitting atomic mass data with conventional Skyrme forces, where a high-precision fit of masses of open-shell nuclei is not possible without a correct spacing of s.p. states close to the Fermi surface. However,

TABLE VII. Properties of nuclear matter at saturation as calculated using the Skyrme parametrizations consistent with the macroscopic constraints. All entries are in $\mathrm{MeV}$ except for dimensionless $\frac{\mathcal{S}\left(\rho_{\mathrm{o}} / 2\right)}{J}, \frac{3 P_{\mathrm{PNM}}}{L \rho_{\mathrm{o}}}$, and $m^{*}$.

\begin{tabular}{|c|c|c|c|c|c|c|c|c|c|c|}
\hline Model & $\rho_{\mathrm{o}}$ & $E_{\mathrm{o}}$ & $K_{\mathrm{o}}$ & $K^{\prime}$ & $J$ & $L$ & $K_{\mathrm{vs}}$ & $\frac{\mathcal{S}\left(\rho_{0} / 2\right)}{J}$ & $\frac{3 P_{\mathrm{PNM}}}{L \rho_{\mathrm{o}}}$ & $m^{*}$ \\
\hline GSkI & 0.159 & -16.02 & 230.21 & 405.58 & 32.03 & 63.45 & -364.19 & 0.62 & 1.02 & 0.78 \\
\hline GSkII & 0.159 & -16.12 & 233.40 & 398.73 & 30.49 & 48.63 & -366.54 & 0.65 & 1.09 & 0.79 \\
\hline LNS & 0.175 & -15.32 & 210.78 & 382.55 & 33.43 & 61.45 & -384.55 & 0.63 & 1.06 & 0.83 \\
\hline MSL0 & 0.160 & -16.00 & 230.00 & 380.32 & 30.00 & 60.00 & -360.11 & 0.61 & 1.06 & 0.80 \\
\hline Ska35s20 & 0.158 & -16.08 & 240.27 & 378.65 & 33.57 & 64.83 & -407.11 & 0.62 & 1.02 & 1.00 \\
\hline SKRA & 0.159 & -15.78 & 216.98 & 378.76 & 31.32 & 53.04 & -364.92 & 0.65 & 1.09 & 0.75 \\
\hline SkT1 & 0.161 & -15.98 & 236.16 & 383.52 & 32.02 & 56.18 & -380.68 & 0.64 & 1.03 & 1.00 \\
\hline SkT2 & 0.161 & -15.94 & 235.73 & 382.67 & 32.00 & 56.16 & -380.48 & 0.64 & 1.03 & 1.00 \\
\hline SkT3 & 0.161 & -15.95 & 235.74 & 382.70 & 31.50 & 55.31 & -374.14 & 0.64 & 1.03 & 1.00 \\
\hline Skxs20 & 0.162 & -15.81 & 201.95 & 425.56 & 35.50 & 67.06 & -383.37 & 0.63 & 1.03 & 0.96 \\
\hline
\end{tabular}


the choice of $M^{*}=M$ is inconsistent, for example, in the context of formation of nuclear matter from isolated nuclei in neutron stars or core-collapse supernova matter. One possible remedy is to construct an extended Skyrme force [119]. More recently, Satula et al. [124] studied the problem of the effective mass scaling within the Skyrme energy density functional (EDF) method. They concluded that more detailed modeling of the two-body spin-orbit and tensor interaction strength reinstates the conventional $m^{*}=M^{*} / M$ scaling and removes the inconsistency in the effective mass scaling of s.p. level densities in nuclear matter and finite nuclei, caused mainly by fitting strategies of the Skyrme parameters to an incomplete Skyrme functional.

Based on the theoretical concept of the effective mass and the experimental data on GQR and GDR we find a strong enough reason to eliminate all CSkP parametrizations with $M^{*}=M$. This choice is not valid in nuclear matter (the prime concern of this work) and has only an auxiliary character in finite nuclei which is likely to be improved upon. The constraint on $m_{s}^{*}$ alone would eliminate the Ska and SkT family forces from the list of CSkP, thereby reducing their number to eleven. For the remaining, $m_{v}^{*}$ at saturation density is calculated in the range $0.603-0.930$. Due to the weak nature of this constraint, deduced from experimental GDR, we do not feel there is a strong enough reason to do any further elimination.

Next, we examine the density dependence of the effective neutron (Fig. 7, left panel) and proton (Fig. 7, right panel) masses, $m_{n}^{*}$ and $m_{p}^{*}$, in BEM. These are very important in modeling cold neutron stars. It can be seen that the set SV-sym32 yields a $m_{n}^{*}$ value close to one at very low density, and this value rises rapidly with increasing density up to about 1.7 at $3 \rho_{0}$. Such behavior is not physical and is a reason for elimination of SV-sym32 from CSkP. We note that some other members of the SV family [115]-SVsym28, SV-sym34, SV-K218, SV-K226, SV-K241, SV-bas, SV-kap60, and SV-mas10 - also exhibit the same feature. They
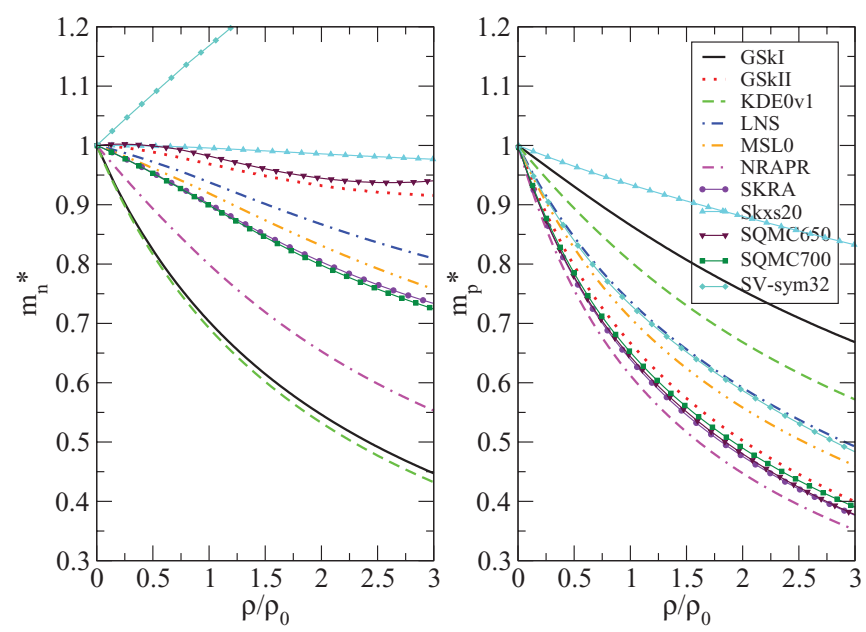

FIG. 7. (Color online) Density dependence of neutron (left panel) and proton (right panel) effective mass in $\beta$-equilibrium matter as calculated by Skyrme interactions consistent with the macroscopic constraints. For more detail see text. all pass through a singularity [98] at densities $\sim 5 \rho_{\mathrm{o}}$ and higher.

\section{B. Landau parameters}

As an alternative to the Hartree-Fock approach to the properties of nuclear matter, the formalism of Landau theory of a normal liquid has been used. In this approach, the bulk properties of nuclear matter are written in terms of a two-body interaction expressed as a functional second derivative of the energy per particle with respect to occupational numbers at the Fermi surface. This has the form $[126,127]$

$$
\begin{aligned}
V_{i, j}= & \delta\left(r_{i}-r_{j}\right) N_{0}^{-1} \sum_{L}\left[F_{L}+F_{L}^{\prime}\left(\tau_{i} \cdot \tau_{j}\right)+G_{L}\left(\sigma_{i} . \sigma_{j}\right)\right. \\
& \left.+G_{L}^{\prime}\left(\tau_{i} \cdot \tau_{j}\right)\left(\sigma_{i} \cdot \sigma_{j}\right)\right] P_{L}(\cos \theta)
\end{aligned}
$$

where $\theta$ is the angle between the momenta of the interacting particles (holes). The number of states per unit energy per unit volume at the Fermi surface in symmetric matter is $N_{0}=\frac{2 M^{*}}{\hbar^{2}} \frac{k_{F}}{\pi^{2}}$, where $k_{F}$ is the Fermi momentum. In pure neutron matter this quantity is halved. The sum is over angular momentum $L$; for the Skyrme interaction, $L=0$ and 1 as it contains only $S$ and $P$ wave contributions. The dimensionless parameters $F$ and $F^{\prime}$ are directly related to quantities describing nuclear matter such as effective mass, incompressibility, symmetry energy, and the speed of sound through relationships $[54,128]$

$$
\begin{aligned}
m_{s}^{*} & =1+\frac{1}{3} F_{1}, \\
K & =3 \frac{\hbar^{2} k_{F}^{2}}{M_{s}^{*}}\left(1+F_{0}\right), \\
\mathcal{S} & =\frac{\hbar^{2} k_{F}^{2}}{6 M_{s}^{*}}\left(1+F_{0}^{\prime}\right), \\
v_{s} & =\frac{\hbar^{2} k_{F}^{2}}{3 M} \frac{1+F_{0}}{1+1 / 3 F_{1}} .
\end{aligned}
$$

The Landau parameters $G$ and $G^{\prime}$ determine, to leading order, properties of nuclear matter in the spin and spin-isospin channels. We note that only six, out of the eight Landau parameters in SNM, are independent, because of two Pauli principle sum rules [129], and conventionally $F_{1}^{\prime}$ and $G_{1}^{\prime}$ are expressed as a function of the other six. In PNM, with no isospin degrees of freedom, only four parameters, $F_{0}, G_{0}, F_{1}$, and $G_{1}$, are nonzero.

It can be established that stability demands each of $F_{L}$, $F_{L}^{\prime}, G_{L}$, and $G_{L}^{\prime}$ to be greater than $-(2 L+1)$ [127]; i.e., $L=0$ parameters must be greater than -1 and $L=1$ terms must be greater than -3 (see Figs. 8 and 9). The most obvious justification for these conditions is the requirement that incompressibility, symmetry energy (for a stable HF solution for symmetric nuclear matter), and speed of sound be positive [see Eqs. (52), (53), and (54)].

An exact relation between parameters of the Skyrme interaction and Landau parameters can be derived (see, e.g., [54,128]). Beside the expressions (51)-(54) for $F_{0}, F_{1}$, and $F_{0}^{\prime}$, we also present the remaining Landau parameters, $G$ and $G^{\prime}$, 

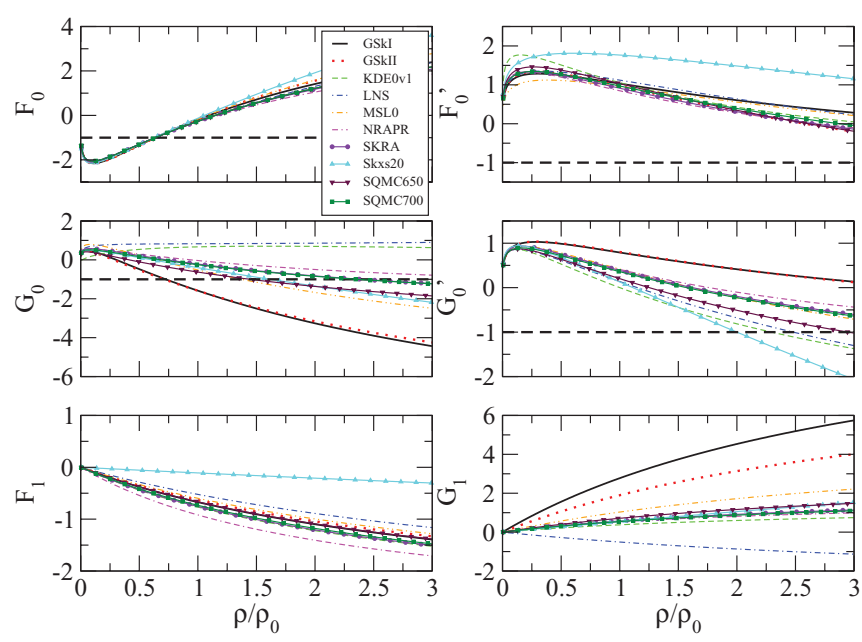

FIG. 8. (Color online) Landau parameters calculated by CSkP sets, which passed microscopic constraints derived from the effective mass considerations, in SNM. See text for more explanation.

given by

$$
\begin{aligned}
G_{0}= & N_{0}\left[\frac{t_{0}}{4}\left(2 x_{0}-1\right)+\frac{1}{24} \sum_{i=1}^{3} t_{3 i}\left(x_{3 i}-1\right) \rho^{\sigma}+\right. \\
& +\frac{t_{1}}{8}\left(2 x_{1}-1\right) k_{F}^{2}+\frac{t_{2}}{8}\left(2 x_{2}+1\right) k_{F}^{2} \\
& \left.+\frac{t_{4}}{8}\left(2 x_{4}-1\right) k_{F}^{2} \rho^{\delta}+\frac{t_{5}}{8}\left(2 x_{5}+1\right) k_{F}^{2} \rho^{\gamma}\right] \equiv G_{0}^{\mathrm{SNM}}, \\
G_{0}^{\prime}= & N_{0}\left[-\frac{t_{0}}{4}-\frac{1}{24} \sum_{i=1}^{3} t_{3 i} \rho^{\sigma}-\frac{t_{1}}{8}\left(2 x_{1}+1\right) k_{F}^{2}\right. \\
& +\frac{t_{2}}{8}\left(2 x_{2}+1\right) k_{F}^{2}-\frac{t_{4}}{8}\left(2 x_{4}+1\right) k_{F}^{2} \rho^{\delta} \\
& \left.+\frac{t_{5}}{8}\left(2 x_{5}+1\right) k_{F}^{2} \rho^{\gamma}\right] \equiv G_{0}^{\prime \mathrm{SNM}},
\end{aligned}
$$
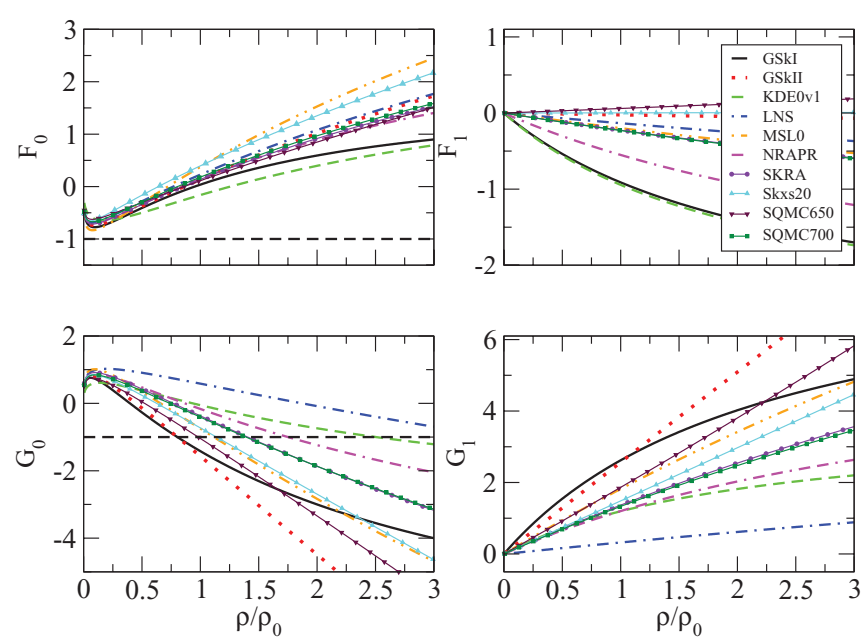

FIG. 9. (Color online) The same as Fig. 8, but for PNM.

$$
\begin{aligned}
G_{1}= & -N_{0} k_{F}^{2}\left[\frac{t_{1}}{8}\left(2 x_{1}-1\right)+\frac{t_{2}}{8}\left(2 x_{2}+1\right)+\frac{t_{4}}{8}\left(2 x_{4}-1\right) \rho^{\delta}\right. \\
& \left.+\frac{t_{5}}{8}\left(2 x_{5}+1\right) \rho^{\gamma}\right] \equiv G_{1}^{\mathrm{SNM}}
\end{aligned}
$$

for SNM [52] and

$$
\begin{aligned}
& G_{0}=G_{0}^{\mathrm{SNM}}+G_{0}^{\mathrm{SNM}}, \\
& G_{1}=G_{1}^{\mathrm{SNM}}+\frac{N_{0} k_{F}^{2}}{8}\left(t_{1}-t_{2}+t_{4} \rho^{\delta}-t_{5} \rho^{\gamma}\right)
\end{aligned}
$$

for PNM [130]; these are also valid for the nonstandard Skyrme models.

Figure 8 (for SNM) and Fig. 9 (for PNM) show the results of the variation of the Landau parameters with density for the CSkP sets, after application of the constraint related to effective mass. It can be seen, at densities below about $\sim 0.1 \mathrm{fm}^{-3}$, that all CSkP sets predict negative incompressibility in SNM. This feature, referred to as a spinodal instability, should be seen as a shortcoming but also a realistic consequence of the strong correlations between nucleons which, at low density, cause them to form a kind of gas-liquid separation. This instability was observed experimentally in heavy-ion collisions [131] at critical densities of about half to two-thirds of nuclear saturation density. No such transition is predicted in PNM (Fig. 9). This instability in symmetric matter and BEM has been consistently predicted by a variety of nonrelativistic HF and RMF models [132].

The density dependence of the parameter $G_{0}$ can be used as an indicator of a breakdown of spin symmetry, i.e., a transition to a spin-ordered (ferromagnetic) phase in SNM as well as in PNM. Such a transition could have important consequences for the evolution of a proton-neutron star in a core-collapse supernova and neutrino transport inside the star [133], but this is not as yet constrained experimentally. Theoretical studies of the spin-ordered phase yield rather contradictory results. Skyrme interactions predict such a transition at low densities (below 3 $\rho_{\mathrm{o}}$ ) in PNM, SNM, and ANM [133-137]. Relativistic DBHF calculations with an effective Lagrangian also predict a transition to a spin-ordered phase at several times $\rho_{\mathrm{o}}$ (see [138] and references therein). In contrast, realistic $N N$ interactions suppress such a transition up to high densities in BHF models [139,140], the AFDMC method [141], and the lowest order constrained variational method [142].

As shown in Fig. 8, the transition to spin-ordered matter is predicted in SNM at densities below $\rho_{\mathrm{o}}$ for GSkI and GSkII parametrizations and at around $1.5 \rho_{\mathrm{o}}$ for MSL0, Skxs20, and SQMC650 parametrizations. In PNM, five parametrizations predict the transition at densities below and around $\rho_{\mathrm{o}}$, GSkI, GSkII, MSL0, Skxs20, and SQMC650, and two at around $1.5 \rho_{\mathrm{o}}$, SKRA and SQMC700.

These features are not realistic and we eliminate GSkI, GSkII, MSL0, Skxs20, and SQMC650 in their present form from the CSkP list as they did not satisfy both the SNM and PNM constraints. However, as demonstrated by Margueron and Sagawa [56], the spin-spin and spin-isospin instabilities can be removed if additional density-dependent terms, affecting only the spin and spin-isospin channels, are included in 
the standard Skyrme Hamiltonian. The contribution of the new terms to the mean field is zero for spin-saturated systems. Consequently the properties of the original Skyrme interaction are not changed in this case. However, in nuclear matter, four new parameters have to be adjusted to values of Landau parameters at saturation density extracted from the $G$ matrix. This procedure is rather involved and is beyond the scope of the present work. We suggest that parametrizations SKRA and SQMC700 would be the best candidates for future application of this treatment [56].

We note that the density dependence of the parameter $G_{0}^{\prime}$, indicates a spin-isospin instability if $G_{0}^{\prime}$ falls below -1 . Such an instability has been interpreted as the appearance of a pion condensate [143,144]. Of the remaining parametrizations, only KDE0v1 and LNS predict such transition in SNM below $3 \rho_{\mathrm{o}}$. All the other Landau parameters are within the natural constraints.

\section{Density dependence of the symmetry energy}

One rather surprising result, which came out of our analysis, is that the CSkP exhibit a growing spread in density dependence of the symmetry energy beyond about twice the nuclear saturation density. This feature is illustrated in Fig. 10 and Table VIII and suggests that constraining the derivatives of the symmetry energy at the saturation point is not sufficient for controlling the slope of $\mathcal{S}(\rho)$ at higher densities. Clearly, more experimental data are needed to constrain the Skyrme interaction at supersaturation densities.

It turns out that, by considering the symmetry energy being the difference between the energy per particle in pure neutron and symmetric matter (to first order), the factor which mainly determines the behavior of the symmetry energy with increasing density is the pure neutron matter EoS. In Fig. 11 we see energy per particle in PNM and SNM as

TABLE VIII. Selected properties of nuclear matter as predicted by consistent Skyrme parametrizations at $3 \rho_{0} . L\left(\rho_{0}\right)$ is included for a comparison. All quantities are in $\mathrm{MeV}$ except for $m^{*}$, which is dimensionless.

\begin{tabular}{lcccrcr}
\hline \hline Force & $m^{*}$ & $\mathcal{S}$ & $L\left(\rho_{\mathrm{o}}\right)$ & $L\left(3 \rho_{\mathrm{o}}\right)$ & \multicolumn{1}{c}{$K_{\text {sym }}$} & \multicolumn{1}{c}{$Q_{\text {sym }}$} \\
\hline GSkI & 0.536 & 60.92 & 63.45 & 89.71 & -253.92 & 947.56 \\
GSkII & 0.557 & 36.42 & 48.63 & -69.49 & -731.84 & 1226.76 \\
KDE0v1 & 0.493 & 55.31 & 54.69 & 53.66 & -192.75 & 1366.50 \\
LNS & 0.612 & 54.15 & 61.45 & 23.06 & -515.88 & 1027.81 \\
MSL0 & 0.571 & 53.91 & 60.00 & 50.80 & -424.29 & 776.89 \\
NRAPR & 0.430 & 53.38 & 59.63 & 27.72 & -468.17 & 1024.00 \\
Ska25s20 & 0.942 & 58.41 & 63.81 & 51.87 & -411.53 & 1047.02 \\
Ska35s20 & 1.000 & 57.75 & 64.83 & 43.51 & -473.55 & 1027.22 \\
SKRA & 0.748 & 44.07 & 53.04 & -22.54 & -596.94 & 1093.69 \\
SkT1 & 1.000 & 48.17 & 56.18 & -0.31 & -527.96 & 1158.54 \\
SkT2 & 1.000 & 48.17 & 56.16 & -0.14 & -527.25 & 1157.11 \\
SkT3 & 1.000 & 47.53 & 55.31 & 0.78 & -515.94 & 1134.50 \\
Skxs20 & 0.899 & 61.70 & 67.06 & 56.83 & -428.23 & 1058.16 \\
SQMC650 & 0.779 & 33.65 & 52.92 & -76.28 & -173.15 & 349.74 \\
SQMC700 & 0.507 & 49.98 & 59.06 & -6.52 & -603.34 & 1109.31 \\
SV-sym32 & 0.749 & 44.14 & 57.07 & -42.43 & -759.99 & 1060.52 \\
\hline \hline
\end{tabular}

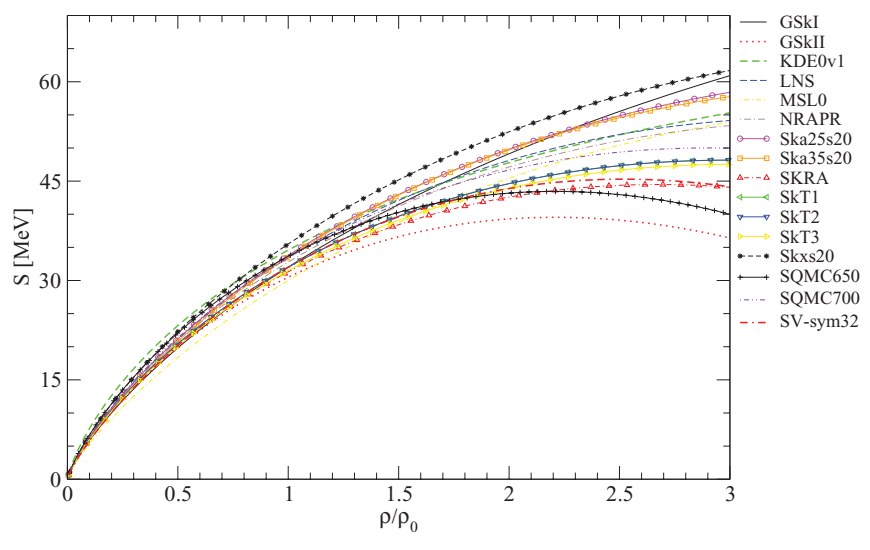

FIG. 10. (Color online) Density dependence of the symmetry energy $\mathcal{S}$ as a function of $\frac{\rho}{\rho_{0}}$ as calculated by Skyrme interactions consistent with macroscopic constraints.

a function of density as calculated with Skxs20, QMC700, and GSkII parametrizations. These forces were chosen as they correspond to the top, middle, and bottom curves in Fig. 10. We see clearly that the energy per particle curves for SNM are rather similar in all three panels, but for PNM they exhibit systematically different patterns. In a sense this is not surprising. Skyrme parametrizations are usually fitted to properties of nuclei with either $N=Z$ or a low value of isospin. The EoS for PNM is well constrained at low densities; at supersaturation densities we have to rely on theoretical models or seek indirect evidence from astrophysical extrapolations, e.g., to neutron stars. So, constraining the PNM EoS by study of very neutron rich heavy nuclei should be desirable.

\section{High-mass cold neutron stars}

One possibility is to use the Skyrme EoS in cold neutron star models up to $3 \rho_{\mathrm{o}}$. Here the Skyrme interaction is applied to $n+$ $p+e+\mu \mathrm{BEM}$ rather then symmetric or pure neutron matter.
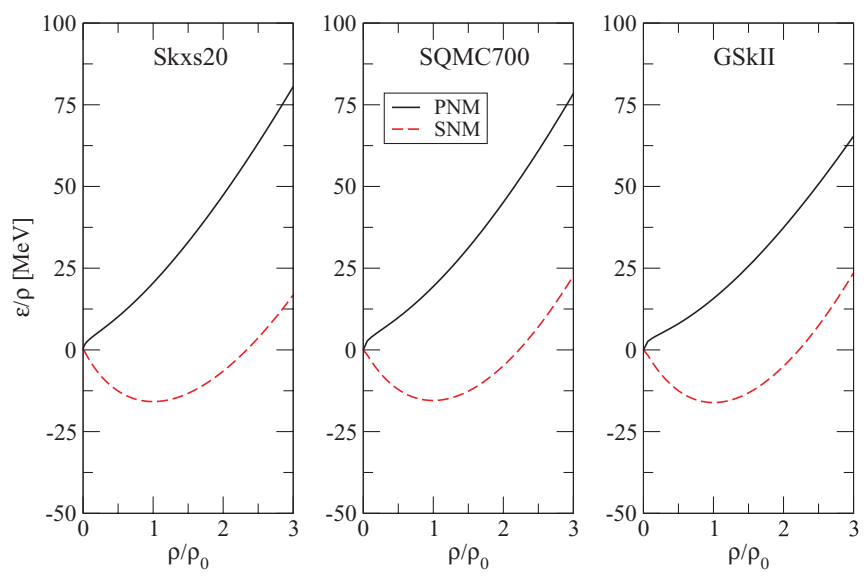

FIG. 11. (Color online) Energy per particle in PNM and SNM as a function of particle number density $\rho$ for three selected Skyrme parametrizations, Skxs20, SQMC700, and GSkII. 

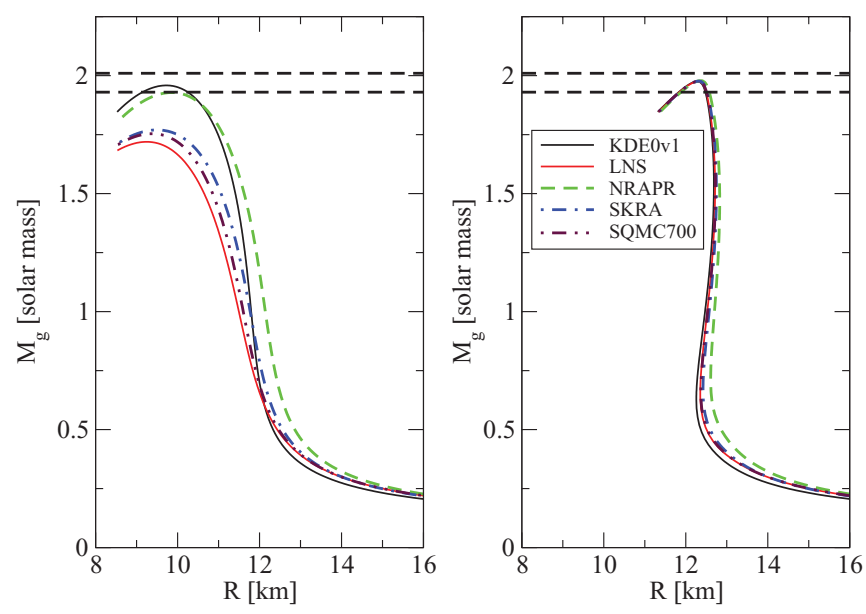

FIG. 12. (Color online) Gravitational mass vs radius for cold nonrotational neutron stars as calculated using a Skyrme EoS augmented by BPS EoS at low density [146] (left panel) and matched by a FQMC EoS at high densities [149] and by BPS EoS at low densities (right panel). The dashed lines indicate the limits on the maximum mass of the most massive neutron star observed up to date [147]. Only Skyrme parametrizations which are consistent with both macroscopic and microscopic constraints are used. For more explanation see text.

There are no constraints available from terrestrial experiments at present, as the heavy-ion reactions are too fast to build equilibrium conditions with respect to weak interactions such as $p+e^{-} \leftrightarrow p+\mu^{-}$.

The findings of Ref. [98] indicate that, if one accepts validity of the Skyrme interaction at densities up to 10 times nuclear saturation density, only parametrizations predicting growing (or monotonically slowly decreasing-for details see Ref. [98]) symmetry energy with increasing density can be used to generate stable neutron star models with mass and radius consistent with currently available observational data [145]. We observe the same phenomenon here for selected CSkP, as illustrated in the left panel of Fig. 12. Here the mass-radius relation for neutron star models was calculated using a BEM Skyrme EoS; since only a part of the neutron star is in the BEM phase, we used the Baym-Pethick-Sutherland (BPS) EoS [146] at lower densities, matching the Skyrme-based part at $\rho \sim 0.1 \mathrm{fm}^{-3}$ and going down to $\rho \sim 6.0 \times 10^{-12} \mathrm{fm}^{-3}$. The observed spread in the maximum mass models is not unexpected; it is related to the different extrapolation properties of the Skyrme interaction to densities well beyond the validity of the Skyrme model. A very recent observational finding of a massive neutron star [147] with $M_{g}=1.97 \pm 0.04 M_{\odot}$ and a central density of less than $\approx 10 \rho_{\mathrm{o}}$ provides a strong constraint on the EoS of BEM. This constraint would certainly eliminate SKRA, SQMC700, and LNS equations of state, all of which predict lower maximum mass models. Moreover, the central densities of all maximum mass neutron star models predicted by CSkP, including those within the window set by the results of Demorest et al. for gravitational mass, predict higher central densities than is allowed (in the region for $11 \rho_{\mathrm{o}}-13 \rho_{\mathrm{o}}$ ). We thus conclude that extrapolation of the Skyrme model beyond its validity range to high densities does not predict cold neutron stars, in agreement with the recent observation. We do not eliminate any Skyrme interaction from the CSkP list on the basis of this constraint because it requires extension of the Skyrme model outside its validity range.

An alternative is to use the Skyrme interaction within its validity range argued in this paper (up to about $3 \rho_{\mathrm{o}}$ ) to construct the EOS of BEM and match it to an established high-density EoS, as well as with the BPS EoS at lower densities. Such an EoS was usually thought to be the BetheJohnson EoS [148] based of the Reid potential and including hyperons in the composite matter at high densities. However, the Demorest et al. observation rules out this EoS as it predicts maximum mass of the neutron star models to be only $\approx 1.85 M_{\odot}$ with central density $\approx 12 \rho_{\mathrm{o}}$. Therefore we use the full quark-meson-coupling (FQMC) model [149] which includes a full baryon octet in the high-density matter and provides high-mass neutron star models in agreement with observation, as shown in the right panel of Fig. 12. The maximum mass is clearly determined by the FQMC model and only the variations in radii of smaller neutron star models with smaller central densities are due to different Skyrme equations of state. Observational data on neutron star radii are very poor at present. However, new observational techniques are being developed and radii may be known within a few percent in the near future. They could be then used as a useful constraint on Skyrme parametrization performance in a high-density neutron-rich environment.

\section{E. Low-mass cold neutron stars}

Observation of the double pulsar J0737-3039 provides another, stringent, constraint on the neutron star EoS and its interpretation by Podsiadlowski et al. [150], and hence on the effective nucleon-nucleon interaction in stellar matter. This constraint is important, as the central density of pulsar B is only $2 \rho_{\mathrm{o}}-3 \rho_{\mathrm{o}}$ and thus the Skyrme interaction is expected to be fully applicable.

The constraint concerns the ratio of the gravitational mass of pulsar B to its baryonic mass. The gravitational mass of pulsar B is very precisely known: $M_{\mathrm{g}}=1.249 \pm 0.001 M_{\odot}$. Estimates of the baryonic mass depend upon its detailed mode of formation. If pulsar B was formed from a white dwarf with an $\mathrm{O}-\mathrm{Ne}-\mathrm{Mg}$ core in an electron-capture supernova, with no or negligible loss of baryonic mass during the collapse, the newly born neutron star will have the same baryonic mass as the precollapse core of the progenitor star. As modeled by Podsiadlowski et al. [150], the baryonic mass of pulsar $\mathrm{B}$ is then between $1.366 M_{\odot}$ and $1.375 M_{\odot}$. The range reflects uncertainties in modeling of the progenitor core such as electron-capture rates, nuclear network calculations, and Coulomb and general relativity corrections. Another simulation of the same process by Kitaura et al. [151] gave $1.360 M_{\odot} \pm 0.002 M_{\odot}$, where the range of the result was mainly due to uncertainty in the EoS and the estimated small mass loss during the collapse.

For any neutron star matter EoS the relation between the gravitational and baryonic mass can be calculated. Figure 13 


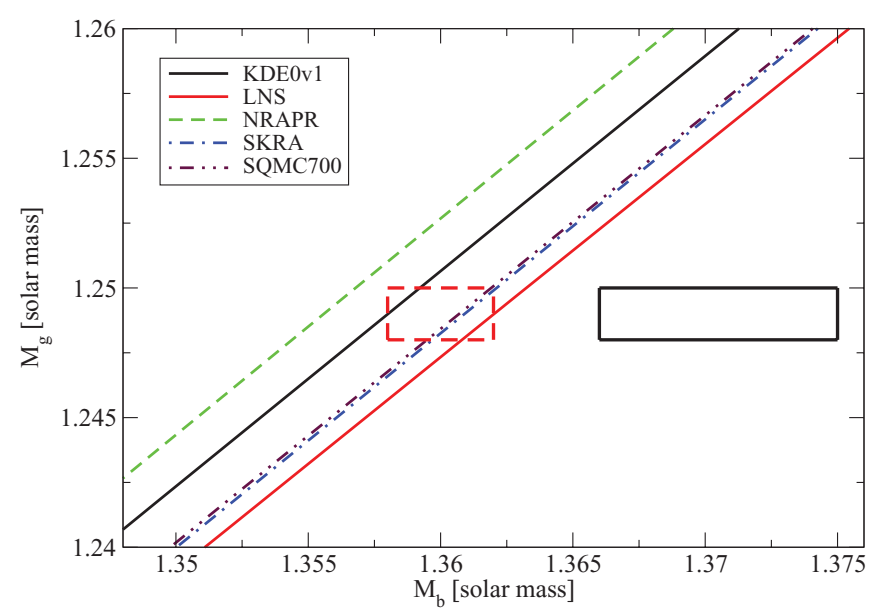

FIG. 13. (Color online) Relation between the gravitational mass, $M_{\mathrm{g}}$, for the selected Skyrme models, and the corresponding baryonic mass, $M_{\mathrm{b}}$. The boxes represent constraints derived by Podsiadlowski et al. [150] (full line box) and more recently by Kitaura et al. [151] (dashed line box) based on the proposed properties of system J0737-3039, as discussed in the text. Results are shown for a Skyrme interaction consistent with both macroscopic and microscopic constraints.

shows the results for the remaining five CSkP to be checked against narrow windows given by the two models of pulsar B. All of the CSkP but NRAPR, which is slightly shifted, agree remarkably well with the result of Kitaura et al. [151], thus supporting the concept of some baryonic mass loss during the collapse.

\section{DISCUSSION AND SUMMARY}

In embarking on this project we hoped the present work might lead to better understanding of how a universal Skyrme force might best be achieved. In this section we discuss the degree to which this hope has been accomplished. A distinction was made between standard and nonstandard forms of the Skyrme interaction, the latter including additional terms added relatively recently. The overall results are discussed below for the two forms.

\section{A. Standard parametrizations}

Only 6 of the over 224 standard sets satisfied all the constraints. Examination of Table III reveals that some families of sets show systematic patterns of failure, as discussed briefly below. Other parametrizations, among them several commonly used for years in modeling finite nuclei, for example, the SIII, SkM*, SkP, SGI, SGII, and the SkI and SKX families, each fail different constraints with no apparent pattern.

This very variable performance makes it rather difficult to identify a particular term or terms in the Skyrme energy functional as responsible. Among the more systematic inconsistencies, BSk1-17 forces, with the exception of BSk14, do not satisfy constraints PNM2, MIX2, and MIX3, a feature shared with most of their predecessors, the SkSC and MSk families. They predict too low a pressure in PNM as a function of increasing supersaturation density. They underpredict both the symmetry energy and its derivative at the saturation density (see Table II and Fig. 6). In addition, the failure of MIX3 indicates too high a volume part of the isospin incompressibility; i.e., they overpredict the isospin dependence of the curvature of the EoS of ANM, $E(\rho, \beta)$, at the saturation density $\rho(\beta)$ [see Eq. (17)].

None of the well-known Lyon forces satisfy constraint MIX3, although they all pass constraint MIX2 (except for SLy10), similarly to the BSk forces. It is interesting to note that the SLy230a force [45], especially developed for modeling of neutron stars, fails constraint PNM1, i.e., it does not have the correct density dependence of pressure at subnuclear densities, which is especially important in modeling of neutron star crusts. The overprediction of $K_{\tau, \mathrm{v}}$ is also a feature of a recently developed extensive set of new Skyrme forces [152] using a fitting protocol similar to that used for construction of Lyon forces.

The failure of MIX3 may seem to be a minor defect but it is persistent and points to the isospin part of the Skyrme force. The isospin dependence of the curvature of the EoS of ANM plays an important role in modeling of giant resonances and heavy-ion collisions [96,108]

The very recently developed model MSL [101], which is expressed in terms of nine macroscopic observables that are either constrained experimentally or well known empirically, offers another nontraditional approach to the construction of a Skyrme parametrization. It expresses the standard Skyrme parameters in terms of these observables and provides a parametrization MSL0 that complies with all but one of the constraints studied in this work. It predicts spin instability around nuclear saturation density, which would be a problem, especially in using this force to model neutron stars.

The successful CSkP sets, several of which were unfortunately infrequently used, do not share much common ground. Their individual parameters, listed in Table VI, are too spread out to give any useful guidance for construction of a more general "consistent" set.

In recent years some effort, aimed at giving more physical relevance to particular terms in the Skyrme energy functional, has led to its re-expression in terms of the coupling constants (some of them density dependent) involving linear combinations of the individual parameters in Eq. (1). Four of these coupling constants, relevant for calculations of nuclear matter, are

$$
\begin{aligned}
& C_{\mathrm{o}}^{\rho}=\frac{3}{8} t_{0}+\frac{3}{48} t_{3} \rho_{\mathrm{o}}^{\sigma}, \\
& C_{1}^{\rho}=-\frac{1}{4} t_{0}\left(\frac{1}{2}+x_{0}\right)-\frac{1}{24} t_{3}\left(\frac{1}{2}+x_{3}\right) \rho_{\mathrm{o}}^{\sigma}, \\
& C_{\mathrm{o}}^{\tau}=\frac{3}{16} t_{1}+\frac{1}{4} t_{2}\left(\frac{5}{4}+x_{2}\right), \\
& C_{1}^{\tau}=-\frac{1}{8} t_{1}\left(\frac{1}{2}+x_{1}\right)+\frac{1}{8} t_{2}\left(\frac{1}{2}+x_{2}\right) .
\end{aligned}
$$

Numerical values of these coupling constants at saturation density are given for CSkP in Table IX. Again, unfortunately, they do not exhibit any apparent regularity. 
TABLE IX. Relevant combinations of Skyrme parameters of consistent Skyrme parametrizations: $C_{\mathrm{o}}^{\rho^{\prime}}$ and $C_{1}^{\rho^{\prime}}$ are given in $\mathrm{MeV} f \mathrm{f}^{3} ; C_{\mathrm{o}}^{\tau^{\prime}}$, and $C_{1}^{\tau^{\prime}}$ are in $\mathrm{MeV} \mathrm{fm}^{5}$; and $\Theta_{\mathrm{s}}^{\prime}, \Theta_{\mathrm{v}}^{\prime}, \Theta_{\mathrm{sym}}^{\prime}$, and $\Theta_{\mathrm{n}}^{\prime}$ are in $\mathrm{MeV} \mathrm{fm}^{5}$ [45]. For full explanation, see text.

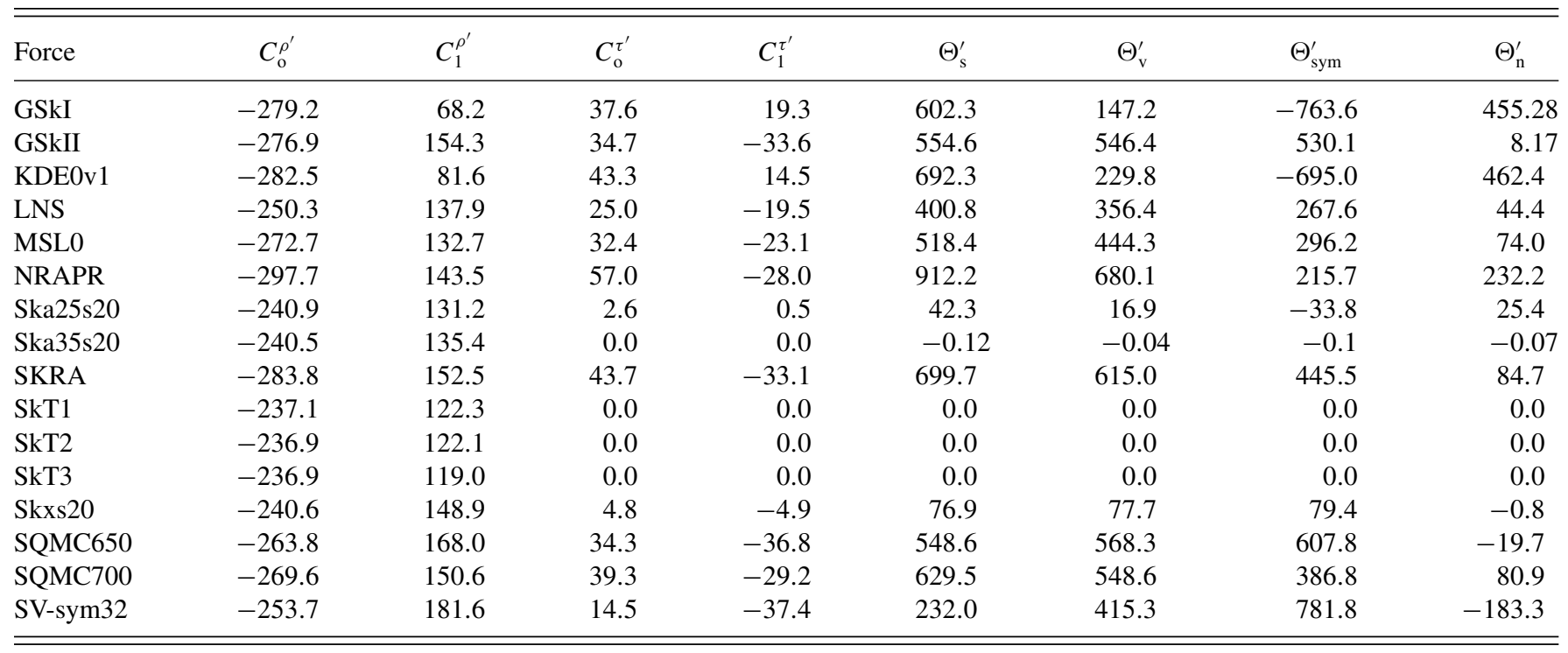

Another combination of parameters, involving only $t_{1}, t_{2}$, $x_{1}$, and $x_{2}$, has been introduced [45]:

$$
\begin{aligned}
\Theta_{\mathrm{s}} & =3 t_{1}+\left(5+4 x_{2}\right) t_{2}, \\
\Theta_{\mathrm{v}} & =t_{1}\left(2+x_{1}\right)+t_{2}\left(2+x_{2}\right), \\
\Theta_{\mathrm{sym}} & =3 t_{1} x_{1}-t_{2}\left(4+5 x_{2}\right), \\
\Theta_{\mathrm{n}} & =t_{1}\left(1-x_{1}\right)+3 t_{2}\left(1+x_{2}\right) .
\end{aligned}
$$

$\Theta_{\mathrm{s}}$ and $\Theta_{\mathrm{v}}$ were used in Sec. IV A in connection with the effective mass. $\Theta_{\text {sym }}$ is used in calculation of the symmetry energy and $\Theta_{\mathrm{n}}$ appears in the expression for the EoS of pure neutron matter. Numerical values, given in Table IX for the CSkP, again show a large scatter.

We are forced to the conclusion that our analysis of the performance of the standard parametrizations in nuclear matter does not offer any clear direction for the development of a unified, generally applicable Skyrme parametrization. Indeed, the overwhelming impression, however well intentioned, is that they are merely empirical attempts to describe nuclearmatter-related phenomena. We see the problem as lying, first, with the lack of a direct connection between the terms of the Skyrme energy functional and specific physics observables and, second, with the strong correlations between the parameters.

\section{B. Nonstandard parametrizations}

In addition to the standard definition of the Skyrme energy density functional, some recently developed Skyrme models include higher order terms, thus introducing additional parameters. The standard form (for application in nuclear matter) depends on 9 parameters, $t_{j}, x_{j}$ (with $j=0-3$ ), and $\sigma$, whereas the extended Skyrme models considered in this work have $t_{3 i}$ and $\sigma_{3 i}$ (with $i=2,3$ ) and $t_{4}$ and $t_{5}$ in addition, totaling 15 adjustable parameters.
Out of all Skyrme models we analyzed, 16 are nonstandard, and some of those share their origin with the standard ones and are closely related to them. For example, the BSk family consists of 22 individual models, 4 of them (BSk18-21) being nonstandard. The BSk18 model behaves as a standard at SNM, since the contribution of the additional terms included adds up to zero. The extended BSk parametrizations were generated by Goriely et al. to improve calculation of nuclear masses at the neutron drip line for $Z, N \geqslant 8$ and $Z \leqslant 120$. The terms containing $t_{4}$ and $t_{5}$ were introduced to ensure a better description for homogeneous neutron matter [130]. With the same aim, and by adding to the conventional Skyrme forces higher order density terms in the EoS $\left[t_{32}\right.$ and $t_{4}$ contributions of Eq. (1)], Farine et al. suggested the SkPS.1 force [119]. These authors claim that this force fits well the nuclear masses of spherical nuclei, and it may be useful to describe stellar collapse processes. Following the same protocol of the "Saclay-Lyon" group (SLy-forces), and by using a better control regarding the spin-isospin instabilities via Landau parameters, Lesinski et al. [153] have also developed three nonstandard forces $\left(f_{-}, f_{0}\right.$, and $\left.f_{+}\right)$. They have attempted to constrain the effective neutron mass in such a way that $m_{n}^{*}<m_{p}^{*}, m_{n}^{*}=m_{p}^{*}$, and $m_{n}^{*}>m_{p}^{*}$, respectively, for $f_{-}$, $f_{0}$, and $f_{+}$. By keeping the $t_{4}$ term in the EoS, Eq. (1), and aiming at a good description of excited states of finite nuclei, Krewald et al. suggested six new nonstandard Skyrme parametrizations: GS1-6 [154,155]. Agrawal et al. [51], by exploring the extended density-dependent Skyrme effective forces for normal and isospin-rich nuclei for neutron stars, parameterized two nonstandard Skyrme forces (GSkI and GSkII) by adjusting $t_{32}$ and $t_{33}$. These models were able to fit consistently 13 finite nuclei: ${ }^{16} \mathrm{O},{ }^{24} \mathrm{O},{ }^{14} \mathrm{Ca},{ }^{48} \mathrm{Ca}$, ${ }^{48} \mathrm{Ni},{ }^{56} \mathrm{Ni},{ }^{68} \mathrm{Ni},{ }^{78} \mathrm{Ni},{ }^{88} \mathrm{Sr},{ }^{90} \mathrm{Zr},{ }^{100} \mathrm{Sn},{ }^{132} \mathrm{Sn}$, and ${ }^{208} \mathrm{~Pb}$. The breathing modes for ${ }^{90} \mathrm{Zr}$ and ${ }^{208} \mathrm{~Pb}$ were also well described. 
TABLE X. Brief compilation of methods used in fitting of the Skyrme interactions consistent with the macroscopic constraints and main data used in the fit. For full explanation and details, see the original papers.

\begin{tabular}{|c|c|c|}
\hline Force & Method & Data used for fit \\
\hline GSkI,GSkII & $\begin{array}{l}\text { Simulated annealing } \\
\text { method }\end{array}$ & $\begin{array}{l}\text { Ground-state properties of normal and exotic nuclei: } \\
\text { binding energies, charge radii, } \\
\text { radii for } 1 d_{5 / 2} \text { and } 1 f_{7 / 2} \text { neutron orbits in }{ }^{17} \mathrm{O} \text { and }{ }^{41} \mathrm{Ca} \text {, } \\
\text { breathing mode energies for }{ }^{90} \mathrm{Zr} \text { and }{ }^{208} \mathrm{~Pb} \text {, } \\
\text { single-particle energies in }{ }^{208} \mathrm{~Pb} \text {, } \\
\text { EoS of pure neutron matter [209] }\end{array}$ \\
\hline MSL0 & MSL [108] model & $\begin{array}{l}\text { Nuclear matter properties: } \\
\rho_{\mathrm{o}}, E_{\mathrm{o}}, K_{\mathrm{o}}, m_{\mathrm{s}, \mathrm{o}}^{*}, m_{\mathrm{v}, \mathrm{o}}^{*} \\
J, L, G_{\mathrm{S}}, G_{\mathrm{V}}[101] \text {; surface symmetry energy, } \\
\text { Landau parameters } G_{\mathrm{o}}\left(\rho_{\mathrm{o}}\right), G_{1}\left(\rho_{\mathrm{o}}\right), G_{\mathrm{o}}^{\prime}\left(\rho_{\mathrm{o}}\right), G_{1}^{\prime}\left(\rho_{\mathrm{o}}\right)\end{array}$ \\
\hline NRAPR & $\begin{array}{l}\text { APR and Skyrme EoS } \\
\text { of NM (a comparison) }\end{array}$ & $\begin{array}{l}\text { Density dependence of effective masses as predicted by APR, } \\
\text { spin-orbit splitting from charge radii and binding energies of }{ }^{208} \mathrm{~Pb},{ }^{90} \mathrm{Zr},{ }^{90} \mathrm{Ca}\end{array}$ \\
\hline $\begin{array}{l}\text { Ska25s20 } \\
\text { Ska35s } 20\end{array}$ & Hartree-Fock & $\begin{array}{l}\text { Binding energy, charge radii, and single-particle energies } \\
\text { Friedman-Pandharipande EoS }\end{array}$ \\
\hline SKRA & $\begin{array}{l}\text { Brueckner-Hartree-Fock } \\
\text { with relativistic corrections } \\
\text { and 3-body forces }\end{array}$ & $\begin{array}{l}\text { Nuclear matter properties: } \\
\rho_{\mathrm{o}}, E_{\mathrm{o}}, K_{\mathrm{o}}, \\
\text { ground-state properties of finite nuclei }\end{array}$ \\
\hline SQMC650,700 & $\begin{array}{l}\text { Quark-meson } \\
\text { coupling model } \\
\text { Hartree-Fock }\end{array}$ & $\begin{array}{l}\text { Comparison for the Skyrme (SkM*) and QMC Hamiltonians; } \\
\text { free parameter: mass of the } \sigma \text { meson: } 650 \text { and } 700 \mathrm{MeV}\end{array}$ \\
\hline SV-sym32 & Hartree-Fock & $\begin{array}{l}\text { Properties of finite nuclei (energies, radii, and surface thickness), } \\
\text { energies of giant resonances, } \\
\text { systematic variations of selected nuclear matter properties }\end{array}$ \\
\hline
\end{tabular}

From all the above-listed Skyrme nonstandard forces, only two, namely, GSkI and GSkII, satisfied the macroscopic constraints but failed the microscopic ones, namely the value of the Landau parameter $G_{0}$. Inclusion of any nonstandard piece in the energy density functional, Eq. (1), inevitably affects parameters of the standard part as both contributions have to be compensated to fit experimental data. Nevertheless, it is instructive to investigate the nonstandard contribution to the energy per particle of symmetric matter, obtained from Eq. (1) and shown in Fig. 14. We see that nonstandard terms may either increase repulsion by a positive term in the effective Skyrme force or increase attraction by a negative term. It is interesting to notice that both GSkI and GSkII forces receive very similar large negative contribution from the nonstandard terms apparently needed to compensate repulsion coming from the standard part of the interaction. However, because of this delicate balance between the standard and nonstandard terms it is difficult to find any general trend. 


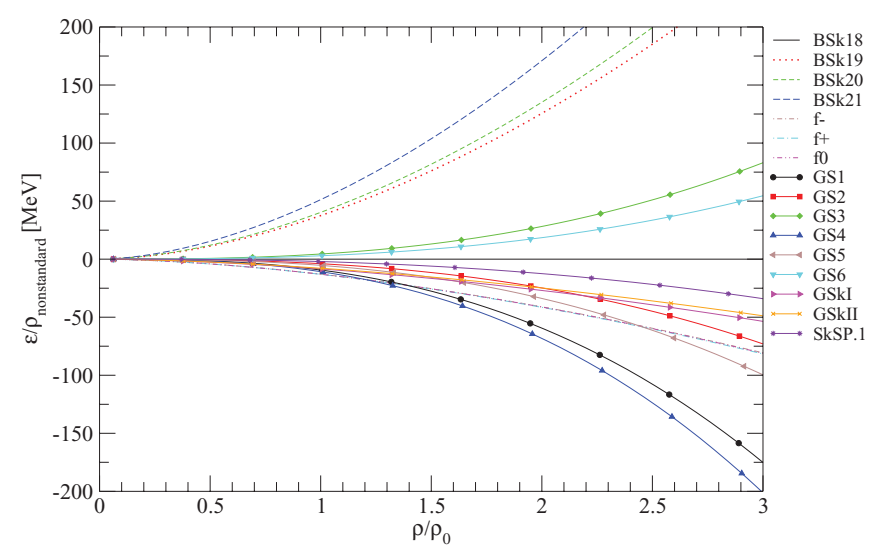

FIG. 14. (Color online) Density dependence of the energy per particle, resulting only from the contributions of the nonstandard terms in Eq. (1) for all nonstandard Skyrme parametrizations used in this work.

As in the case of the standard Skyrme parametrizations, one can also define the following set of coupling constants [43]:

$$
\begin{aligned}
C_{\mathrm{o}}^{\rho^{\prime}}= & \frac{3 t_{0}}{8}+\frac{3}{48} \sum_{i=1}^{3} t_{3 i} \rho_{\mathrm{o}}^{\sigma_{i}}, \\
C_{1}^{\rho^{\prime}}= & -\frac{t_{0}}{4}\left(\frac{1}{2}+x_{0}\right)-\frac{1}{24} \sum_{i=1}^{3} t_{3 i} \rho_{\mathrm{o}}^{\sigma_{i}}\left(\frac{1}{2}+x_{3 i}\right), \\
C_{\mathrm{o}}^{\tau^{\prime}}= & \frac{3 t_{1}}{16}+\frac{t_{2}}{4}\left(\frac{5}{4}+x_{2}\right)+\frac{3 t_{4}}{16} \rho_{\mathrm{o}}^{\delta}+\frac{t_{5}}{4}\left(\frac{5}{4}+x_{5}\right) \rho_{\mathrm{o}}^{\gamma}, \\
C_{1}^{\tau^{\prime}}= & -\frac{t_{1}}{8}\left(\frac{1}{2}+x_{1}\right)+\frac{t_{2}}{8}\left(\frac{1}{2}+x_{2}\right)-\frac{t_{4}}{8}\left(\frac{1}{2}+x_{4}\right) \rho_{\mathrm{o}}^{\delta} \\
& +\frac{t_{5}}{8}\left(\frac{1}{2}+x_{5}\right) \rho_{\mathrm{o}}^{\gamma},
\end{aligned}
$$

written as a function of the nonstandard parameters. Notice that these equations are generalized forms of Eqs. (60). The same occurs for the quantities [45]

$$
\begin{aligned}
\Theta_{\mathrm{s}}^{\prime} & =\Theta_{\mathrm{s}}+3 t_{4} \rho^{\delta}+t_{5}\left(5+4 x_{5}\right) \rho^{\gamma}, \\
\Theta_{\mathrm{v}}^{\prime} & =\Theta_{\mathrm{v}}+t_{4}(2+x 4) \rho^{\delta}+t_{5}\left(2+x_{5}\right) \rho^{\gamma}, \\
\Theta_{\mathrm{sym}}^{\prime} & =\Theta_{\mathrm{sym}}+3 t_{4} x_{4} \rho^{\delta}-t_{5}\left(4+5 x_{5}\right) \rho^{\gamma}, \\
\Theta_{\mathrm{n}}^{\prime} & =\Theta_{\mathrm{n}}+t_{4}\left(1-x_{4}\right) \rho^{\delta}+3 t_{5}\left(1+x_{5}\right) \rho^{\gamma} .
\end{aligned}
$$

All these values are shown in Table IX.

Although it may be useful to extend the Skyrme functional to improve results in particular physical situation, it does not seem to be a way forward to finding a recipe for getting the Skyrme model under control.

\section{CONCLUSIONS}

We have examined the performance of 240 different Skyrme model parametrizations in nuclear matter by comparing their predictions of behavior in eleven areas in which experimentally and empirically derived constraints exist. The chosen macroscopic constraints cover a wide range of properties related to symmetric nuclear matter (SM1-SM4), pure neutron matter (PNM1 and PNM2), and both SNM and PNM (MIX1MIX5). Of the Skyrme models, 6 satisfy all the constraints while 66 satisfy all but one. For 10 of the 66 the single failure is narrow, less then 5\%. Including these yields a final list of 16 consistent models, the CSkP set GSkI, GSkII, KDE0v1, LNS, MSL0, NRAPR, Ska25s20, Ska35s20, SKRA, Skxs20, SQMC650, SQMC700, SkT1, SkT2, SkT3, and SV-sym32. The parameters of these interactions are summarized in Table X.

As an additional step, we considered four microscopic constraints arising from giant resonance experiments on nucleon effective mass, Landau parameters in SNM and PNM, and observation of low-mass neutron stars. With these microscopic constraints taken into account the successful set reduces to five, KDE0v1, LNS, NRAPR, SKRA, and SQMC700, the $\mathrm{CSkP}^{*}$ set.

A fifth microscopic constraint, maximum mass and the corresponding central density of high-mass neutron stars, creates a fundamental obstacle to applying Skyrme (nucleon only) models in neutron star modeling since it requires extrapolation to densities above the range of validity. None of the CSkP models produce a maximum mass neutron star model with central density in line with observation. Thus if this constraint were applied, all CSkP would fail.

We were unable to identify any regularities, either in single parameters or in their combinations, to identify a unique quality of the CSkP* sets. This is hardly surprising when we consider the number of parameters and their correlations, and it is tempting to suggest that some of the sets satisfied all the constraints by a fortunate accident. This looks particularly likely in the case of the KDE family. Of very close KDE0 parameter sets (KDE0v0 and KDE0v1), which fit the same experimental data but differ only by starting conditions for the simulated-annealing fit procedure, only KDE0v1 passes our constraints. It may be significant that in this procedure particular attention was paid to the inclusion of nuclear matter quantities in the fit. Also energies of the giant monopole resonances were included directly to the fit for the first time.

The example of KDE0v1 indicates that the inclusion of as many constraints as possible, both macroscopic and microscopic, in the fitting protocols of the Skyrme interaction is essential. For example, we believe that the symmetry energy plays a key role in the behavior of nuclear matter. Therefore the correct determination of the PNM EoS is imperative. Although there are no direct experimental or observational data on PNM at supersaturation densities, promising $a b$ initio theoretical predictions and indirect experimental data from cold atoms at subsaturation densities are becoming available. Any further development in this area is very desirable.

It may be also revealing that in construction of three out of five CSkP* parametrizations, LNS, NRAPR, and SKRA, the EoS of nuclear matter, used in the fit, was derived from realistic potentials. Such an approach amplifies the role of the microscopic physics input in the effective Skyrme interaction model. The usual practice of including basic properties of nuclear matter only at saturation density is not sufficient. The 
density dependence of these observables (within the range of applicability of the Skyrme model), which may considerably influence the fits, must be included.

The last parametrization on the CSkP* list, SQMC700, is unique in its derivation from the full quark-meson-coupling model [149,156,157], which includes the full baryon octet at high densities and is relativistic. The structure of the QMC Hamiltonian and its Skyrme equivalent (FQMC limit at low densities) are somewhat different, which shows up most markedly in the difference between the values of the incompressibility for the FQMC EoS [149] and QMC forces considered in this paper. Nevertheless, the fact that in the FQMC the many-body interaction is directly related to the response of the quark structure to the nuclear environment may cause the very simple QMC Skyrme parametrization, with $x_{1}=x_{2}=x_{3}=0$ and $\sigma=1 / 6$, to be consistent with all the constraints.

It is important to stress that our present work is restricted to examining the performance of the Skyrme interaction in nuclear-matter-related scenarios. It is generally true that only a limited effort was spent to make the CSkP* perform well in wide-ranging application in finite nuclei when they were derived in the original papers. In some sense, this may be a positive factor as it seems obvious that Skyrme interactions, constructed with an emphasis on nuclear matter properties, will do better on constraints derived from nuclear matter then interactions heavily biased toward properties of finite nuclei. As a follow-up project of this work, the performance of CSkP in finite nuclei will be studied in more detail [158]. It will be interesting to test the quality of the Skyrme functional by extending the fitting protocol to include not only all the constraints studied in this work but also requirements based on the most up-to-date finite nuclei data, including drip-line and superheavy nuclei. It may turn out that the ambition to fit using such a detailed protocol may be asking too much of the Skyrme model (with its known deficiencies [123]) but the attempt may also lead to a parametrization with increased predictive power. It is our opinion that a parametrization, successful in reproducing only a selected subset of available data but failing the rest, does not have credible predictive power and does not progress the field.

The results obtained in this paper should be seen as the first step in a global effort to find the best possible Skyrme interactions for use in modeling of nuclear matter. We intend to monitor developments both in keeping up to date the existing constraints and in adopting new ones. Progress in ab initio calculations of inhomogeneous neutron matter [159] and chiral effective field theory $[15,16]$ are good examples of sources of new constraints which will be taken into account in the future. New experimental results on giant resonances, neutron skin in heavy nuclei, and heavy-ion collisions, as well as new astrophysical observations, will further improve the set of benchmark constraints, which may shed more light on the structure and applicability of the Skyrme interaction. Should new Skyrme parameter sets appear in the literature, we intend to catalog and test them using the most complete set of constraints available to us.

The outcome of our analysis of all standard and nonstandard parametrizations does not offer a final solution to the "Skyrme proliferation" problem. Neither does it provide general guidance for construction of more Skyrme parameter sets. The production of new parameter sets having limited ranges of application should not be encouraged. Rather, more emphasis should be put on better understanding the existing models, which should be further tested against an extended number of refined constraints including both nuclear matter and finite-nuclei-related properties, with equal emphasis. The Skyrme interaction has played a dominant role in low-energy nuclear physics for decades. The approach suggested can lead to a final judgment of whether or not this interaction includes enough physics not only for a successful interpretation but also for a prediction of the rich variety of data and observations on nuclear and astrophysical systems available today and expected in the future.

\section{ACKNOWLEDGMENTS}

The authors thank Betty Tsang for providing Fig. 6 prior to publication. JRS acknowledges helpful discussions with Anthony Thomas, Pierre Guichon, Steven Moszkowski, P.-G. Reinhard, Bijay Agrawal, Shalom Shlomo, and Nick Stone. This work was supported by the Brazilian agencies $\mathrm{CNPq}$, CAPES, and FAPESP. JRS has the pleasure to thank Debora Menezes's group at Universidade Federal Santa Catarina for its hospitality during her stay, made possible by $\mathrm{CNPq}$ Processo (401593/2009-6). She also acknowledges with thanks the support under UK STFC Grant No. EP/D077133/1 and the stimulating environment at the University of Surrey during the final stages of this work. MD and OL thank the members of the UFSC staff for their hospitality during the initial stage of this work and Debora Menezes for encouragement and support.
[1] V. G. J. Stoks, R. A. M. Klomp, C. P. F. Terheggen, and J. J. de Swart, Phys. Rev. C 49, 2950 (1994); R. B. Wiringa, V. G. J. Stoks, and R. Schiavilla, ibid. 51, 38 (1995); L. Engvik, M. Hjorth-Jensen, R. Machleidt, H. Muther, and A. Polls, Nucl. Phys. A 627, 85 (1997); R. Machleidt, Phys. Rev. C 63, 024001 (2001), and references therein.

[2] B. ter Haar and R. Malfliet, Phys. Rep. 149, 207 (1987); E. N. E. van Dalen, C. Fuchs, and A. Faessler, Nucl. Phys. A 741, 227 (2004); F. de Jong and H. Lenske, Phys. Rev. C 57, 3099 (1998).
[3] J. Cugnon, P. Deneye, and A. Lejeune, Z. Phys. A 328, 409 (1987); W. Zuo, A. Lejeune, U. Lombardo, and J. F. Mathiot, Eur. Phys. J. A 14, 469 (2002); I. Bombaci and U. Lombardo, Phys. Rev. C 44, 1892 (1991); B. D. Day and R. B. Wiringa, ibid. 32, 1057 (1985); M. Baldo, G. F. Burgio, and H.-J. Schulze, ibid. 61, 055801 (2000); I. Vidaña, A. Polls, A. Ramos, L. Engvik, and M. Hjorth-Jensen, ibid. 62, 035801 (2000).

[4] A. Akmal, V. R. Pandharipande, and D. G. Ravenhall, Phys. Rev. C 58, 1804 (1998). 
[5] A. Mukherjee and V. R. Pandharipande, Phys. Rev. C 75, 035802 (2007).

[6] A. Fabricioni and S. Fantoni, Phys. Lett. B 298, 263 (1993); C. Bisconti, F. A. de Saavedra, G. Co, and A. Fabrocini, Phys. Rev. C 73, 054304 (2006).

[7] Y. Dewulf, W. H. Dickhoff, D. Van Neck, E. R. Stoddard, and M. Waroquier, Phys. Rev. Lett. 90, 152501 (2003).

[8] T. Frick and H. Müther, Phys. Rev. C 68, 034310 (2003).

[9] B. S. Pudliner, V. R. Pandharipande, J. Carlson, S. C. Pieper, and R. B. Wiringa, Phys. Rev. C 56, 1720 (1997).

[10] K. E. Schmidt and S. Fantoni, Phys. Lett. B 446, 99 (1999).

[11] J. Carlson, J. Morales Jr., V. R. Pandharipande, and D. G. Ravenhall, Phys. Rev. C 68, 025802 (2003).

[12] S. Gandolfi, F. Pederiva, S. Fantoni, and K. E. Schmidt, Phys. Rev. Lett. 99, 022507 (2007).

[13] S. Gandolfi, A. Yu. Illarionov, S. Fantoni, F. Pederiva, and K. E. Schmidt, Phys. Rev. Lett. 101, 132501 (2008).

[14] S. Gandolfi, A. Yu. Illarionov, K. E. Schmidt, F. Pederiva, and S. Fantoni, Phys. Rev. C 79, 054005 (2009).

[15] K. Hebeler and A. Schwenk, Phys. Rev. C 82, 014314 (2010).

[16] K. Hebeler, J. M. Lattimer, C. J. Pethick, and A. Schwenk, Phys. Rev. Lett. 105, 161102 (2010).

[17] Z. H. Li, U. Lombardo, H.-J. Schulze, W. Zuo, L. W. Chen, and H. R. Ma, Phys. Rev. C 74, 047304 (2006).

[18] P. Haensel, M. Proszynski, and M. Kutschera, Astron. Astrophys. 102, 299 (1981).

[19] F. Coester, S. Cohen, B. D. Day, and C. M. Vincent, Phys. Rev. C 1, 769 (1970).

[20] G. Q. Li, R. Machleidt, and R. Brockmann, Phys. Rev. C 45, 2782 (1992).

[21] B. D. Serot and J. D. Walecka, Adv. Nucl. Phys. 16, 1 (1986).

[22] A. Delfino, J. B. Silva, and M. Malheiro, Phys. Rev. C 73, 037311 (2006).

[23] H. S. Köhler, Phys. Rev. 137, B1145 (1965); 138, B831 (1965).

[24] D. M. Brink and E. Boeker, Nucl. Phys. A 91, 1 (1967).

[25] S. A. Moszkowski, Phys. Rev. C 2, 402 (1970).

[26] T. H. R. Skyrme, Phil. Mag. 1, 1043 (1956).

[27] D. Vautherin and D. M. Brink, Phys. Rev. C 5, 626 (1972).

[28] J. Decharge and D. Gogny, Phys. Rev. C 21, 1568 (1980).

[29] P. Stevenson, M. R. Strayer, and J. R. Stone, Phys. Rev. C 63, 054309 (2001).

[30] J. R. Stone, P. D. Stevenson, J. C. Miller, and M. R. Strayer, Phys. Rev. C 65, 064312 (2002).

[31] W. D. Myers and W. J. Świątecki, Ann. Phys. 55, 395 (1969).

[32] W. D. Myers and W. J. Świątecki, Ann. Phys. 84, 186 (1974).

[33] C. J. Pethick and D. G. Ravenhall, Annu. Rev. Nucl. Part. Sci. 45, 429 (1995).

[34] G. Röpke, M. Schmidt, L. Münchow, and H. Schultz, Nucl. Phys. A 399, 587 (1983).

[35] S. Heckel, P. P. Schneider, and A. Sedrakian, Phys. Rev. C 80, 015805 (2009).

[36] D. G. Ravenhall, C. J. Pethick, and J. R. Wilson, Phys. Rev. Lett. 50, 2066 (1983); C. J. Horowitz, M. A. Perez-Garcia, and J. Piekarewicz, Phys. Rev. C 69, 045804 (2004); C. J. Horowitz, M. A. Perez-Garcia, D. K. Berry, and J. Piekarewicz, ibid. 72, 035801 (2005); T. Maruyama, T. Tatsumi, D. N. Voskresensky, T. Tanigawa, and S. Chiba, ibid. 72, 015802 (2005).

[37] S. S. Avancini, D. P. Menezes, M. D. Alloy, J. R. Marinelli, M. M. W. Moraes, and C. Providência, Phys. Rev. C 78, 015802
(2008); S. S. Avancini, L. Brito, J. R. Marinelli, D. P. Menezes, M. M. W. de Moraes, C. Providência, and A. M. Santos, ibid. 79, 035804 (2009).

[38] W. G. Newton and J. R. Stone, Phys. Rev. C 79, 055801 (2009).

[39] A. Schwenk and C. J. Pethick, Phys. Rev. Lett. 95, 160401 (2005).

[40] E. Epelbaum, H. Krebs, D. Lee, and U.-G. Meissner, Eur. Phys. J. A 40, 199 (2009).

[41] A. Gezerlis and J. Carlson, Phys. Rev. C 77, 032801 (2008).

[42] A. Gezerlis and J. Carlson, Phys. Rev. C 81, 025803 (2010).

[43] M. Bender, P.-H. Heenen, and P.-G. Reinhard, Rev. Mod. Phys. 75, 121 (2003).

[44] J. R. Stone and P. G. Reinhard, Prog. Part. Nucl. Phys. 58, 587 (2007).

[45] E. Chabanat, P. Bonche, P. Haensel, J. Meyer, and R. Schaeffer, Nucl. Phys. A 627, 710 (1997).

[46] M. Dutra, O. Lourenço, A. Delfino, J. S. Sá Martins, C. Providência, S. S. Avancini, and D. P. Menezes, Phys. Rev. C 77, 035201 (2008).

[47] M. Dutra, O. Lourenço, A. Delfino, and J. S. Sá Martins, Int. J. Mod. Phys. E 16, 3006 (2007); Int. J. Mod. Phys. D 19, 1583 (2010).

[48] A. Sulaksono, T. Büervenich, J. A. Maruhn, P.-G. Reinhard, and W. Greiner, Ann. Phys. 308, 354 (2003).

[49] O. Lourenço, M. Dutra, A. Delfino, and J. S. Sá Martins, Phys. Rev. C 81, 038201 (2010); A. Delfino, O. Lourenço, M. Dutra, and J. S. Sá Martins, Int. J. Mod. Phys. D 19, 1469 (2010).

[50] A. Sulaksono, T. Büervenich, P. O. Hess, and J. A. Maruhn, Int. J. Mod. Phys. E 20, 139 (2011).

[51] B. K. Agrawal, S. K. Dhiman, and R. Kumar, Phys. Rev. C 73, 034319 (2006).

[52] N. Chamel, S. Goriely, and J. M. Pearson, Phys. Rev. C 80, 065804 (2009).

[53] M. Waroquier, J. Sau, K. Heyde, P. Van Isacker, and H. Vincx, Phys. Rev. C 19, 1983 (1979).

[54] K.-F. Liu, Z. Ma, Q. Shen, and S. A. Moszkowski, Nucl. Phys. A 534, 1 (1991).

[55] B. Cochet, K. Bennaceur, P. Bonche, T. Duguet, and J. Meyer, Nucl. Phys. A 731, 34 (2004).

[56] J. Margueron and H. Sagawa, J. Phys. G 36, 125102 (2009).

[57] I. Vidaña, C. Providência, A. Polls, and A. Rios, Phys. Rev. C 80, 045806 (2009).

[58] J. P. Blaizot, Phys. Rep. 64, 171 (1980).

[59] S. Shlomo and D. H. Youngblood, Phys. Rev. C 47, 529 (1993).

[60] J. P. Blaizot, J. F. Berger, J. Decharge, and M. Girod, Nucl. Phys. A 591, 435 (1995).

[61] J. M. Pearson, N. Chamel, and S. Goriely, Phys. Rev. C 82, 037301 (2010).

[62] J. Treiner, H. Krivine, O. Bohias, and J. Martorell, Nucl. Phys. A 371, 253 (1981).

[63] R. C. Nayak, J. M. Pearson, M. Farine, P. Gleissl, and M. Brack, Nucl. Phys. A 516, 62 (1990).

[64] D. H. Youngblood, H. L. Clark, and Y.-W. Lui, Phys. Rev. Lett. 82, 691 (1999).

[65] M. Farine, J. M. Pearson, and F. Tondeur, Nucl. Phys. A 615, 135 (1997).

[66] W. D. Myers and W. J. Świątecki, Phys. Rev. C 57, 3020 (1998).

[67] G. Colò, N. V. Giai, J. Meyer, K. Bennaceur, and P. Bonche, Phys. Rev. C 70, 024307 (2004); Nucl. Phys. A 731, 15 (2004).

[68] B. K. Agrawal, S. Shlomo, and V. Kim Au, Phys. Rev. C 68, 031304 (2003). 
[69] B. G. Todd-Rutel and J. Piekarewicz, Phys. Rev. Lett. 95, 122501 (2005).

[70] T. Li et al., Phys. Rev. Lett. 99, 162503 (2007).

[71] T. Li et al., Phys. Rev. C 81, 034309 (2010).

[72] D. H. Youngblood, Y.-W. Lui, H. L. Clark, B. John, Y. Tokimoto, and X. Chen, Phys. Rev. C 69, 034315 (2004).

[73] Y.-W. Lui, D. H. Youngblood, Y. Tokimoto, H. L. Clark, and B. John, Phys. Rev. C 70, 014307 (2004).

[74] J. Li, G. Colò, and J. Meng, Phys. Rev. C 78, 064304 (2008).

[75] D. Vretenar, T. Niksic, and P. Ring, Phys. Rev. C 68, 024310 (2003).

[76] J. Piekarewicz, J. Phys. G 37, 064038 (2010).

[77] J. Piekarewicz and M. Centelles, Phys. Rev. C 79, 054311 (2009).

[78] P. Danielewicz, R. Lacey, and W. G. Lynch, Science 298, 1592 (2002).

[79] D. Brill et al., Z. Phys. A 355, 61 (1996).

[80] W. G. Lynch, M. B. Tsang, Y. Zhang, P. Danielewicz, M. Famiano, Z. Li, and A. W. Steiner, Prog. Part. Nucl. Phys. 62, 427 (2009).

[81] C. Fuchs, Prog. Part. Nucl. Phys. 56, 1 (2006).

[82] J. Carlson, S.-Y. Chang, V. R. Pandharipande, and K. E. Schmidt, Phys. Rev. Lett. 91, 050401 (2003).

[83] M. Bartenstein, A. Altmeyer, S. Riedl, S. Jochim, C. Chin J. H. Denschlag, and R. Grimm, Phys. Rev. Lett. 92, 120401 (2004).

[84] J. Kinast, A. Turlapov, J. E. Thomas, Q. Chen, J. Stajic, and K. Levin, Science 307, 1296 (2005).

[85] J. T. Stewart, J. P. Gaebler, C. A. Regal, and D. S. Jin, Phys. Rev. Lett. 97, 220406 (2006).

[86] L. Luo and J. E. Thomas, J. Low Temp. Phys. 159, 1 (2009).

[87] M. J. H. Ku, A. T. Sommer, L. W. Cheuk, and M. W. Zwierlein, Science 335, 563 (2012).

[88] S. Gandolfi, K. E. Schmidt, and J. Carlson, Phys. Rev. A 83, 041601(R) (2011).

[89] P. Möller, W. D. Meyers, H. Sagawa, and S. Yoshida, Phys. Rev. Lett. 108, 052501 (2012).

[90] M. B. Tsang, Y. Zhang, P. Danielewicz, M. Famiano, Z. Li, W. G. Lynch, and A. W. Steiner, Phys. Rev. Lett. 102, 122701 (2009).

[91] A. Klimkiewicz et al., Phys. Rev. C 76, 051603 (2007).

[92] Z. Kohley et al., Phys. Rev. C 83, 044601 (2011).

[93] A. Carbone, G. Colò, A. Bracco, L.-G. Cao, P. F. Bortignon, F. Camera, and O. Wieland, Phys. Rev. C 81, 041301 (2010).

[94] O. Wieland et al., Phys. Rev. Lett. 102, 092502 (2009).

[95] P. Danelewicz and J. Lee, arXiv:1111.0326v1.

[96] B.-A. Li, L. W. Chen, and C. M. Ko, Phys. Rep. 464, 113 (2008).

[97] M. B. Tsang, J. R. Stone et al. (private communication).

[98] J. R. Stone, J. C. Miller, R. Koncewicz, P. D. Stevenson, and M. R. Strayer, Phys. Rev. C 68, 034324 (2003).

[99] B. A. Brown, Phys. Rev. Lett. 85, 5296 (2000).

[100] S. Typel and B. A. Brown, Phys. Rev. C 64, 027302 (2001).

[101] L. W. Chen, C. M. Ko, B.-A. Li, and J. Xu, Phys. Rev. C 82, 024321 (2010).

[102] L. W. Chen, C. M. Ko, and B.-A. Li, Phys. Rev. C 72, 064309 (2005).

[103] W. G. Newton and B.-A. Li, Phys. Rev. C 80, 065809 (2009).

[104] W. D. Myers and W. J. Świa̧tecki, Nucl. Phys. A 601, 141 (1996).

[105] U. Garg, Acta Phys. Pol. B 42, 659 (2011).
[106] H. Sagawa, S. Yoshida, G.-M. Zeng, J.-Z. Gu, and X.-Z. Zhang, Phys. Rev. C 76, 034327 (2007).

[107] J. R. Stone, S. A. Moszkowski, and N. J. Stone (private communication).

[108] L. W. Chen, B. J. Cai, C. M. Ko, B.-A. Li, C. Shen, and J. Xu, Phys. Rev. C 80, 014322 (2009).

[109] S. K. Patra, M. Centelles, X. Viñas, and M. Del Estal, Phys. Rev. C 65, 044304 (2002).

[110] P. Danielewicz, Nucl. Phys. A 727, 233 (2003).

[111] J. Piekarewicz, Phys. Rev. C 66, 034305 (2002).

[112] J. Piekarewicz, Phys. Rev. C 76, 064310 (2007).

[113] F. Tondeur, M. Brack, M. Farine, and J. M. Pearson, Nucl. Phys. A 420, 297 (1984).

[114] K. A. Brueckner, Phys. Rev. 97, 1353 (1955).

[115] P. Klüpfel, P.-G. Reinhard, T. J. Bürvenich, and J. A. Maruhn, Phys. Rev. C 79, 034310 (2009).

[116] P. Ring and P. Schuck, The Nuclear Many-Body Problem (Springer-Verlag, New York, 1980).

[117] P.-G. Reinhard (private communication).

[118] L. G. Cao, U. Lombardo, C. W. Shen, and N. Van Giai, Phys. Rev. C 73, 014313 (2006).

[119] M. Farine, J. M. Pearson, and F. Tondeur, Nucl. Phys. A 696, 396 (2001).

[120] The CDFE (Center for Photonuclear Experiments Data) Team, Lomonosov Moscow State University, Skobeltsyn Institute of Nuclear Physics, cdfe.sinp.msu.ru/services (2007).

[121] O. Bohigas, A. M. Lane, and J. Martorelli, Phys. Rep. 51, 267 (1979).

[122] H. Krivine, J. Treiner, and O. Bohigas, Nucl. Phys. A 336, 155 (1980).

[123] J. Erler, P. Klupfel, and P.-G. Reinhard, J. Phys. G 37, 064001 (2010).

[124] W. Satula, R. A. Wyss, and M. Zalewski, Phys. Rev. C 78, 011302(R) (2008).

[125] V. Bernard and N. Van Giai, Nucl. Phys. A 348, 75 (1980).

[126] S.-O. Bäckman, C.-G. Källman, and O. Sjöberg, Phys. Lett. B 43, 263 (1973).

[127] S.-O. Bäckman, A. D. Jackson, and J. Speth, Phys. Lett. B 56, 209 (1975).

[128] J. Margueron, J. Navarro, and N. Van Giai, Phys. Rev. C 66, 014303 (2002).

[129] B. L. Friman and A. K. Dhar, Phys. Lett. B 85, 1 (1979).

[130] S. Goriely, N. Chamel, and J. M. Pearson, Phys. Rev. C 82, 035804 (2010).

[131] E. Suraud, C. Gregoire, and B. Tamain, Prog. Part. Nucl. Phys. 23, 357 (1989).

[132] C. Ducoin, C. Providência, A. M. Santos, L. Brito, and Ph. Chomaz, Phys. Rev. C 78, 055801 (2008).

[133] S. Reddy, M. Prakash, J. M. Lattimer, and J. A. Pons, Phys. Rev. C 59, 2888 (1999).

[134] A. Viduarre, J. Navarro, and J. Bernabeu, Astron. Astrophys. 135, 361 (1984).

[135] A. A. Isayev, JETP Lett. 77, 251 (2003).

[136] A. A. Isayev and J. Yang, Phys. Rev. C 69, 025801 (2004).

[137] A. Rios, A. Polls, and I. Vidaña, Phys. Rev. C 71, 055802 (2005).

[138] P. Bernardos, S. Marcos, R. Niembro, and M. L. Quelle, Phys. Lett. B 356, 175 (1995).

[139] I. Vidaña, A. Polls, and A. Ramos, Phys. Rev. C 65, 035804 (2002).

[140] I. Vidaña and I. Bombaci, Phys. Rev. C 66, 045801 (2002). 
[141] S. Fantoni, A. Sarsa, and K. E. Schmidt, Phys. Rev. Lett. 87, 181101 (2001).

[142] G. H. Bordbar and M. Bigdeli, Phys. Rev. C 77, 015805 (2008).

[143] A. Akmal and V. R. Pandharipande, Phys. Rev. C 56, 2261 (1997).

[144] A. B. Migdal, Rev. Mod. Phys. 50, 107 (1978).

[145] J. M. Lattimer and M. Prakash, Phys. Rep. 442, 109 (2007).

[146] G. Baym, C. Pethick, and P. Sutherland, Astrophys. J. 170, 299 (1971).

[147] P. B. Demorest, T. Pennucci, S. M. Ransom, M. S. E. Roberts, and J. W. T. Hessels, Nature (London) 467, 1081 (2010).

[148] H. A. Bethe and M. Johnson, Nucl. Phys. A 230, 1 (1974).

[149] J. R. Stone, P. A. M. Guichon, H. Matevosyan, and A. W. Thomas, Nucl. Phys. A 792, 341 (2007).

[150] Ph. Podsiadlowski, J. D. M. Dewi, P. Lesaffre, J. C. Miller, W. G. Newton, and J. R. Stone, Mon. Not. R. Astron. Soc. 361, $1243(2005)$.

[151] F. S. Kitaura, H.-Th. Janka, and W. Hillebrandt, Astron. Astrophys. 450, 345 (2006).

[152] T. Lesinski, M. Bender, K. Bennaceur, T. Duguet, and J. Meyer, Phys. Rev. C 76, 014312 (2007).

[153] T. Lesinski, K. Bennaceur, T. Duguet, and J. Meyer, Phys. Rev. C 74, 044315 (2006).

[154] G.-Q. Li, J. Phys. G 17, 1 (1991).

[155] S. Krewald, V. Klemt, J. Speth, and A. Faessler, Nucl. Phys. A 281, 166 (1977).

[156] P. A. M. Guichon and A. W. Thomas, Phys. Rev. Lett. 93, 132502 (2004).

[157] P. A. M. Guichon, H. H. Matevosyan, N. Sandulescu, and A. W. Thomas, Nucl. Phys. A 772, 1 (2006).

[158] P. D. Stevenson et al. (private communication).

[159] S. Gandolfi, J. Carlson, and S. C. Pieper, Phys. Rev. Lett. 106, 012501 (2011).

[160] M. Samyn et al., Nucl. Phys. A 700, 142 (2002).

[161] S. Goriely, M. Samyn, P.-H. Heenen, J. M. Pearson, and F. Tondeur, Phys. Rev. C 66, 024326 (2002).

[162] M. Samyn, S. Goriely, and J. M. Pearson, Nucl. Phys. A 725, 69 (2003).

[163] S. Goriely, M. Samyn, M. Bender, and J. M. Pearson, Phys. Rev. C 68, 054325 (2003).

[164] M. Samyn, S. Goriely, M. Bender, and J. M. Pearson, Phys. Rev. C 70, 044309 (2004).

[165] S. Goriely, M. Samyn, J. M. Pearson, and M. Onsi, Nucl. Phys. A 750, 425 (2005).

[166] S. Goriely, M. Samyn, and J. M. Pearson, Nucl. Phys. A 773, 279 (2006).

[167] S. Goriely, M. Samyn, and J. M. Pearson, Phys. Rev. C 75, 064312 (2007).

[168] S. Goriely and J. M. Pearson, Phys. Rev. C 77, 031301 (2008).

[169] N. Chamel, S. Goriely, and J. M. Pearson, Nucl. Phys. A 812, 72 (2008).

[170] S. Goriely, N. Chamel, and J. M. Pearson, Phys. Rev. Lett. 102, 152503 (2009).

[171] J. Friedrich and P.-G. Reinhard, Phys. Rev. C 33, 335 (1986).

[172] J. Meyer, Lectures at the 11th Joliot-Curie School of Nuclear Physics, Maubuisson, September 1993.

[173] B. K. Agrawal, S. Shlomo, and V. K. Au, Phys. Rev. C 72, 014310 (2005).

[174] F. Tondeur, S. Goriely, J. M. Pearson, and M. Onsi, Phys. Rev. C 62, 024308 (2000).
[175] S. Goriely, M. Pearson, and F. Tondeur, Nucl. Phys. A 688, 349 (2001).

[176] M. M. Sharma, G. Lalazissis, J. Konig, and P. Ring, Phys. Rev. Lett. 74, 3744 (1995).

[177] A. W. Steiner, M. Prakash, J. M. Lattimer, and P. J. Ellis, Phys. Rep. 411, 325 (2005).

[178] S. J. Lee and A. Z. Mekjian, Phys. Rev. C 63, 044605 (2001); H. R. Jaqaman, A. Z. Mekjian, and L. Zamick, ibid. 29, 2067 (1984)

[179] M. Rayet, M. Arnould, F. Tondeur, and G. Paulus, Astron. Astrophys. 116, 183 (1982).

[180] P.-G. Reinhard (private communication).

[181] N. Van Giai and H. Sagawa, Phys. Lett. B 106, 379 (1981).

[182] Q. B. Shen, Y. L. Han, and H. R. Guo, Phys. Rev. C 80, 024604 (2009).

[183] M. Beiner, H. Flocard, N. Van Giai, and P. Quentin, Nucl. Phys. A 238, 29 (1975).

[184] M. J. Giannoni and P. Quentin, Phys. Rev. C 21, 2076 (1980).

[185] C. J. Pethick, D. G. Ravenhall, and C. P. Lorenz, Nucl. Phys. A 584, 675 (1995).

[186] S. Köhler, Nucl. Phys. A 258, 301 (1976).

[187] B. A. Brown (private communication).

[188] P.-G. Reinhard and H. Flocard, Nucl. Phys. A 584, 467 (1995).

[189] W. Nazarewicz, J. Dobaczewski, T. R. Werner, J. A. Maruhn, P.-G. Reinhard, K. Rutz, C. R. Chinn, A. S. Umar, and M. R. Strayer, Phys. Rev. C 53, 740 (1996).

[190] J. Bartel, P. Quentin, M. Brack, C. Guet, and H.-B. Hakansson, Nucl. Phys. A 386, 79 (1982).

[191] J. M. G. Gomez and M. Casas, Few-Body Syst. Suppl. 8, 374 (1995); X. Li and P.-H. Heenen, Phys. Rev. C 54, 1617 (1996).

[192] L. Bennour, P.-H. Heenen, P. Bonche, J. Dobaczewski, and H. Flocard, Phys. Rev. C 40, 2834 (1989).

[193] P.-G. Reinhard, D. J. Dean, W. Nazarewicz, J. Dobaczewski, J. A. Maruhn, and M. R. Strayer, Phys. Rev. C 60, 014316 (1999).

[194] J. Dobaczewski, H. Flocard, and J. Treiner, Nucl. Phys. A 422, 103 (1984).

[195] M. Rashdan, Mod. Phys. Lett. A 15, 1287 (2000).

[196] J. M. G. Gómez, C. Prieto, and J. Navarro, Nucl. Phys. A 549, 125 (1992).

[197] J. M. Pearson, Y. Aboussir, A. K. Dutta, R. C. Nayak, M. Farine, and F. Tondeur, Nucl. Phys. A 528, 1 (1991).

[198] Y. Aboussir, J. M. Pearson, A. K. Dutta, and F. Tondeur, Nucl. Phys. A 549, 155 (1992).

[199] J. M. Pearson and R. C. Nayak, Nucl. Phys. A 668, 163 (2000).

[200] M. Onsi, H. Przysiezniak, and J. M. Pearson, Phys. Rev. C 50, 460 (1994).

[201] R. C. Nayak and J. M. Pearson, Phys. Rev. C 52, 2254 (1995).

[202] C. M. Ko, H. C. Paul, M. Brack, and G. E. Brown, Nucl. Phys. A 236, 269 (1974).

[203] J. Treiner and H. Krivine, J. Phys. G 2, 285 (1976).

[204] B. A. Brown, Phys. Rev. C 58, 220 (1998).

[205] B. A. Brown, G. Shen, G. C. Hillhouse, J. Meng, and A. Trzcińska, Phys. Rev. C 76, 034305 (2007).

[206] E. Chabanat, Ph.D. Thesis, University of Lyon, 1995.

[207] E. Chabanat, P. Bonche, P. Haensel, J. Meyer, and R. Schaeffer, Nucl. Phys. A 635, 231 (1998)

[208] J. M. Pearson and S. Goriely, Phys. Rev. C 64, 027301 (2001).

[209] R. B. Wiringa, V. Fiks, and A. Fabrocini, Phys. Rev. C 38, 1010 (1988) 